\title{
New Frontiers on van der Waals Layered Metal Phosphorous Trichalcogenides
}

\author{
Fengmei Wang, Tofik A. Shifa, Peng Yu, Peng He, Yang Liu, Feng Wang, \\ Zhenxing Wang, Xueying Zhan, Xiaoding Lou,* Fan Xia,* and Jun He*
}

The exponentially growing works on 2D materials have resulted in both high scientific interest and huge potential applications in nanocatalysis, optoelectronics, and spintronics. Of especial note is that the newly emerged and promising family of metal phosphorus trichalcogenides $\left(\mathrm{MPX}_{3}\right)$ contains semiconductors, metals, and insulators with intriguing layered structures and architectures. The bandgaps of the members in this family range from 1.3 to $3.5 \mathrm{eV}$, significantly enriching the application of 2D materials in the broad wavelength spectrum. In this review, emphasizing their remarkable structural, physicochemical, and magnetic properties, as well as the numerous applications in various fields, the innovative progress on layered $\mathrm{MPX}_{3}$ crystals is summarized. Different from other layered materials, these crystals will advance a fascinating frontier in magnetism and spintronic devices with their especially featured atomic layered nanosheets. Thus, their crystal and electronic structures, along with some related researches in magnetism, are discussed in detail. The assortments of growth methods are then summarized. Considering their potential applications, the prominent utilization of these 2D MPX 3 nanoscrystals in catalysis, batteries, and optoelectronics is also discussed. Finally, the outlook of these kinds of layered nanomaterials is provided.

\section{Introduction}

van der Waals layered materials, including graphene, ${ }^{[1]}$ transition metal dichalcogenides (TMDs), ${ }^{[2,3]}$ black phosphorus, ${ }^{[4,5]}$ and boron nitride $(\mathrm{BN}),{ }^{[6]}$ dominate the current era of $2 \mathrm{D}$ crystals. Given their unique physical and chemical properties, which do not appear in 3D structures, they are widely utilized as electronic and structural materials. ${ }^{[7]}$ Especially, the fabrication of heterostructures and optoelectronic nanodevices using 2D materials has been studied, resulting in extraordinary findings in various fields of study. The electron confinement in the 2D feature endows them with some specific properties, such

Dr. F. M. Wang, Dr. T. A. Shifa, P. He, Y. Liu, Dr. F. Wang, X. Zhan,

Prof. Z. Wang, Prof. I. He

CAS Center for Excellence in Nanoscience

CAS Key Laboratory of Nanosystem and Hierarchical Fabrication

National Center for Nanoscience and Technology

Beijing 100190, China

E-mail: hej@nanoctr.cn

The ORCID identification number(s) for the author(s) of this article can be found under https://doi.org/10.1002/adfm.201802151. as charge density wave occurrence, ${ }^{[8]}$ anisotropic magnetic behavior ${ }^{[9]}$ and conductivity, ${ }^{[2,4]}$ anisotropy of photoluminescence, ${ }^{[10]}$ and large surface area. In this regard, TMD materials represent a strikingly indispensable series of 2D crystals. As far as their structure is concerned, strong covalent bonded layers, constituting a cationic plane $\left(\mathrm{M}^{4+}\right)$ enclosed by two planes of anionic $\left(\mathrm{S}^{2-}, \mathrm{Se}^{2-}\right.$, or $\left.\mathrm{Te}^{2-}\right)$, enlarge into infinite sheets. The arrangement of such $\mathrm{S}(\mathrm{Se})-\mathrm{M}-\mathrm{S}(\mathrm{Se})$ sandwiches provides an empty space, in which two adjacent layers are weakly bonded to each other via the van der Waals (vdW) force. ${ }^{[11,12]}$ Their optical and electronic properties could be controlled by changing the components and the thickness. ${ }^{[13,14]}$ For instance, the bandgap of $\mathrm{MoS}_{2}$ (a typical TMD) can be enlarged from 1.29 to $1.9 \mathrm{eV}$ via reducing the thickness from multilayers to a monolayer. ${ }^{[13,15]}$ Although their bandgaps can be tuned, TMDs are generally characterized by small bandgaps (smaller than $2.02 \mathrm{eV}$ ), ${ }^{[16]}$ which limits their application in devices requiring values beyond this range. Thus, 2D materials exhibiting bandgaps ranging from 2.02 to $4.0 \mathrm{eV}$ are extensively sought to meet the application requirements in the broad wavelength spectrum.

Nowadays, a burgeoning research direction goes toward the newly emerged van der Waals layered materials known as metal phosphorus trichalcogenides $\left(\mathrm{MPX}_{3}\right)$. The metal cations $\left(\mathrm{M}^{\mathrm{II}}\right.$ or $\left.\mathrm{M}^{\mathrm{I}} \mathrm{M}^{\mathrm{III}}\right)$ stabilize $\left[\mathrm{P}_{2} \mathrm{~S}_{6}\right]^{4-}$ or $\left[\mathrm{P}_{2} \mathrm{Se}_{6}\right]^{4-}$ framework layers weakly bonding each other via vdW interactions. This family contains a variety of transition metal phases, ranging from vanadium to zinc ( $\mathrm{M}=\mathrm{V}, \mathrm{Mn}, \mathrm{Fe}, \mathrm{Co}, \mathrm{Ni}, \mathrm{Zn}$, etc.) in the first row, partial alkaline metal in group-II, and some other metal

DOI: $10.1002 / \mathrm{adfm} .201802151$

\author{
Dr. T. A. Shifa, P. He, Y. Liu, Prof. J. He \\ University of Chinese Academy of Sciences \\ Beijing 100049, P. R. China \\ P. Yu, Prof. X. Lou, Prof. F. Xia \\ Engineering Research Center of Nano-Geomaterials of Ministry \\ of Education \\ Faculty of Materials Science and Chemistry \\ China University of Geosciences \\ 388 Lumo Road, Wuhan 430074, P. R. China \\ E-mail: louxiaoding@cug.edu.cn, xiafan@cug.edu.cn
}


ions. Friede ${ }^{[17]}$ and Ferrand ${ }^{[18,19]}$ discovered them in the late 1800s. Based on the interesting structure of these materials, significant research works were reported in the early 2000s. As expected, 2D MPX 3 phases share most of the abovementioned specific properties of 2D TMDs. According to the theoretical and experimental results, $\mathrm{MPX}_{3}$ compounds are the most sought functional materials for their intermediate bandgaps ranging from 1.3 to $3.5 \mathrm{eV},{ }^{[20,21]}$ indicating their enhanced light absorption efficiency as compared to the TMD materials. In addition, their unusual intercalation-substitution or intercalation-reduction behavior as well as the incipient ionic conductivity promote their usage in Li-ion batteries, ${ }^{[22,23]}$ gas storage, ${ }^{[24]}$ and photo-electrochemical reactions. ${ }^{[25]}$ Unlike TMDs, several $\mathrm{MPX}_{3}$ materials show intrinsic anti-ferromagnetism below the Neel temperatures of $78 \mathrm{~K}$ for $\mathrm{MnPS}_{3}, 116 \mathrm{~K}$ for $\mathrm{FePS}_{3}$, and $155 \mathrm{~K}$ for NiPS ${ }_{3}{ }^{[26,27]}$ Recently, Li et al. ${ }^{[28]}$ predicted that transformation from the anti-ferromagnetism to ferromagnetism for exfoliated $\mathrm{MnPSe}_{3}$ nanosheet will be reduced by carrier doping. And the Monte Carlo simulation reveals the Curie temperature of the doped $\mathrm{MnPSe}_{3}$ nanosheets can reach $206 \mathrm{~K}$, rendering it with potential for utilizations in spintronic devices at high temperature. Therefore, the members in the $\mathrm{MPX}_{3}$ family have the abovementioned properties along with structural flexibility stemming from their van der Waals nature; thus, it is reasonable to assume that they will contribute to the next major frontier in 2D vdW layered materials.

Herein, we emphasize on reviewing the impressive recent progress and thoughts on physical and chemical characteristics, specific properties, growth methods, and potential applications of $2 \mathrm{D} \mathrm{MPX}$ crystals. The structure of $\mathrm{MPX}_{3}$ is comprehensively described and compared with the common TMD's structure. According to the variety of cations $(\mathrm{M})$ and their corresponding valence states, they are categorized into $\mathrm{M}^{\mathrm{II}}, \mathrm{M}^{\mathrm{I}} \mathrm{M}^{\mathrm{III}}$ and other component-based $\mathrm{MPX}_{3}$ materials. The bandgaps, crystal structures, and stacking of layers are summarized for tangible deduction on the materials functionality and specific properties. Furthermore, the material functionality and specific properties of $\mathrm{MPX}_{3}$ are also discussed. Up to now, the synthesis of $2 \mathrm{D}$ ultrathin $\mathrm{MPX}_{3}$ crystals has been a big challenge. Therefore, we are inclined to summarize the growth of atomic layered $\mathrm{MPX}_{3}$ crystals. Considering the feature-based application of this type of layered crystals, we also introduce some applications. The final section of this review contains future perspectives of these types of 2D layered materials.

\subsection{Composition and Crystal Structures}

All the members in the family of $\mathrm{MPX}_{3}$ materials share a defining common structural feature such that $\left(\mathrm{P}_{2} \mathrm{~S}_{6}\right)^{4-}$ or $\left(\mathrm{P}_{2} \mathrm{Se}_{6}\right)^{4-}$ anion sublattice appears within each layered crystal. The honeycomb arrangement of the transition metal ions is distributed around the $\left(\mathrm{P}_{2} \mathrm{X}_{6}\right)^{4-}$ bipyramids. Thus, these compounds are usually described as $\mathrm{M}_{2} \mathrm{P}_{2} \mathrm{~S}_{6}$. A structural comparison between typical layered $\mathrm{MoS}_{2}$ and $\mathrm{MPX}_{3}$ is shown in Figure 1. In general, the vdW gap (shortest distance between the $\mathrm{S}$ layers) distance of the $\mathrm{MPX}_{3}$ crystals with the first row transition metal elements is around 3.22-3.24 A, much wider than that of $\mathrm{MS}_{2}$ ones. Various atomic radii cations can be

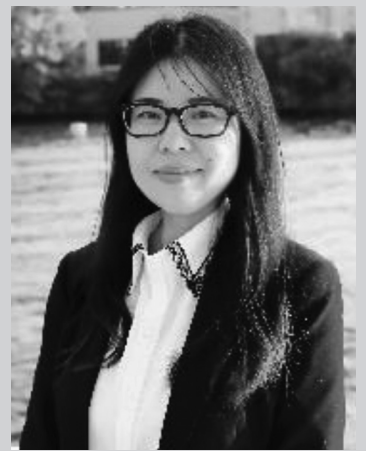

Fengmei Wang received her B.S. degree in applied chemistry from Hunan University, China, in 2012. Then she received her Ph.D. degree in physical chemistry from the University of Chinese Academy of Sciences at the National Center for Nanoscience and Technology (NCNST), China, in 2017. From then on, she worked in NCNST as an assistant professor. Her major research interests include synthesis and development of novel 2D materials, such as transition metal chalcogenides, meal phosphorous trichalcogenides, etc., for electrocatalysis or photocatalysis.

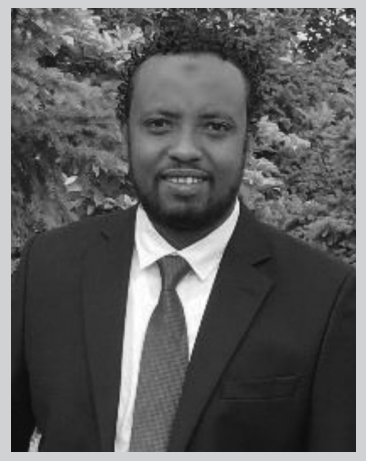

Tofik A. Shifa received his B.S. degree in applied chemistry from Arbaminch University, Ethiopia in 2007. He then obtained his M.S. degree in analytical chemistry from Haramaya University, Ethiopia in 2011. He has recently got a Ph.D. degree in physical chemistry from the University of Chinese Academy of Sciences at the National Center for Nanoscience and Technology (NCNST), Beijing, China. His research focuses on the controllable synthesis of 2D layered materials for water splitting.

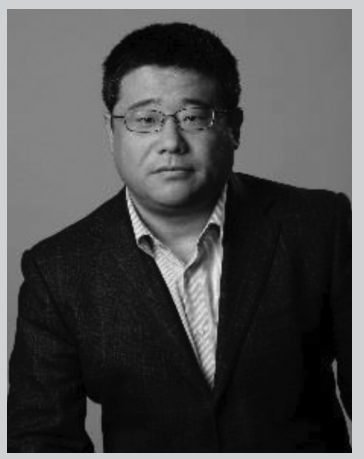

Jun He received his Ph.D. in semiconductor physics from the Institute of Semiconductors, Chinese Academy of Sciences (CAS), in 2003. Then he worked successively at the Applied Physics Department of Technische Universiteit Eindhoven, Netherlands, The Material Department of University of California, Santa Babara, and California NanoSystem Insitute (CNSI), University of California, Los Angeles, USA. He joined the "100-Talents" Program of CAS in November 2010 and became a full professor of NCNST since then. Jun's main research interests are the synthesis, characterization, and devices of low dimensional semiconductor materials. 
a)
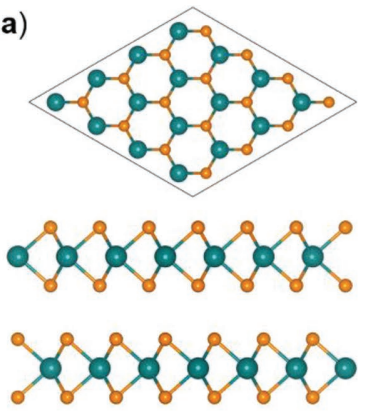

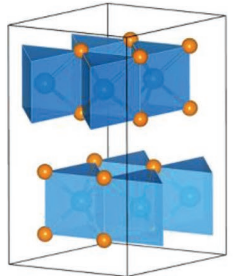

- $\mathrm{Mo} \odot \mathrm{s} / \mathrm{Se}$ b)

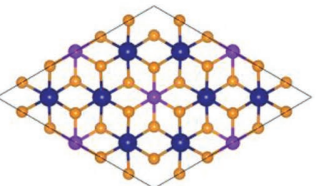

sos

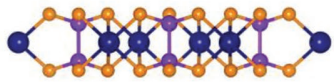

c)

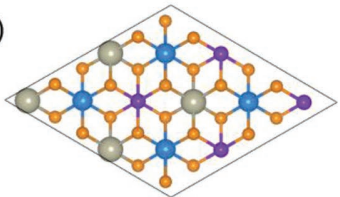

รัง

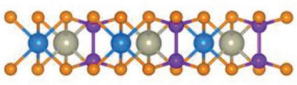

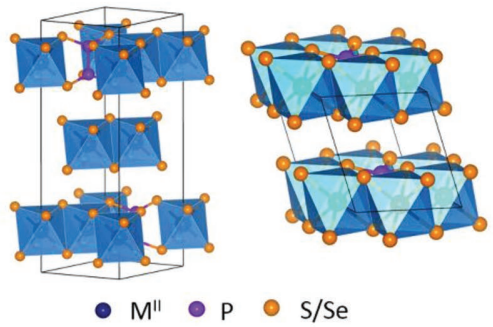

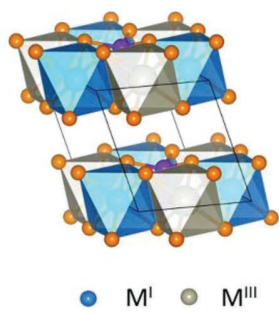

Figure 1. Comparison of crystal structures, including the top view (top), side view with two layers (middle), and crystal phase, of a) MoS $\mathrm{S}_{2}$ with hexagonal crystal, b) $\mathrm{M}^{\prime \prime} \mathrm{PX}_{3}$ with hexagonal (left) and monoclinic(right) crystals, and c) $\mathrm{M}^{\prime} \mathrm{M}^{\prime \prime \prime} \mathrm{PX}{ }_{3}$ with monoclinic crystal. Here, the $2 \mathrm{H}$ phase of MoS 2 is taken as an example.

located in the $\mathrm{MPX}_{3}$ along with the change of the slab size. As to the $\mathrm{P}_{2} \mathrm{~S}_{6}{ }^{4-}$ structural modification, the flat pyramid constituting $\mathrm{S}^{2-}$ ions remains invariable but the $\mathrm{P}-\mathrm{P}$ distance is somewhat elongated to accommodate the different metal cations. There is a shift, regardless of the type of $\mathrm{M}$ cations, from $2.148 \AA\left(\mathrm{NiPS}_{3}\right)$ to $2.222 \AA\left(\mathrm{CdPS}_{3}\right)$ for P-P distance in $\mathrm{MPS}_{3}$ crystals. ${ }^{[29]} \mathrm{How}$ ever, the layer thickness is positively related to the value of the $\mathrm{P}-\mathrm{P}$ distances for $\mathrm{MPX}_{3}$ crystals. ${ }^{[30]}$ The component of $\mathrm{MPX}_{3}$ can be tuned by changing the $\mathrm{M}$ and $\mathrm{X}$ atoms. On the anion side, $\mathrm{P}_{2} \mathrm{~S}_{6}{ }^{4-}$ and $\mathrm{P}_{2} \mathrm{Se}_{6}{ }^{4-}$ anions can be alloyed in the $\mathrm{M}_{2} \mathrm{P}_{2} \mathrm{X}_{6}$ structure. Mixed chalcogen, in which the various atoms (i.e., $\mathrm{S}$ or $\mathrm{Se}$ ) are alloyed with each other, is adopted. Some alloys, such as $\mathrm{Ni}_{2} \mathrm{P}_{2} \mathrm{~S}_{6-x} \mathrm{Se}_{x},{ }^{[31]} \mathrm{CuInP}_{2} \mathrm{~S}_{6-x} \mathrm{Se}_{x},{ }^{[27,32]} \mathrm{MnPS}_{x} \mathrm{Se}_{3-x},{ }^{[33]}$ and $\mathrm{Sn}_{2} \mathrm{P}_{2} \mathrm{~S}_{6-x} \mathrm{Se}_{x},{ }^{[34]}$ have been studied. On the cation side, $\mathrm{MPX}_{3}$ can accommodate group II elements (e.g., Mg), group IV elements (e.g., Sn), transition metals (i.e., Sc, Mn, Fe, Co, Pd, Ag, and $\mathrm{Cd}$, etc.), or a combination thereof. Until now, the most studied materials are $\mathrm{Mn}, \mathrm{Fe}$, and Ni based $\mathrm{MPX}_{3}$ compounds due to their magnetic ordering and applications in electrochemistry and catalysis. ${ }^{[20,35,36]}$ From the component point of view, it constitutes a potentially broad material class. Figure 2 shows metal elements in the periodic table that are known to crystallize into $\mathrm{MPX}_{3}$ type materials. In the following sections, these $\mathrm{MPX}_{3}$ crystals will be classified according to the different cations and described in detail.

\subsection{1. $M^{\prime \prime}$ Based $M P X_{3}$ Crystals}

Divalent metal atom-based $\mathrm{M}^{\mathrm{II}} \mathrm{PX}_{3}$ crystals are common and intensively studied presently. Notably, this aspect is one of particularities to distinguish $\mathrm{M}^{\mathrm{II}} \mathrm{PX}_{3}$ from $\mathrm{M}^{\mathrm{IV}} \mathrm{X}_{2}$ (e.g., $\mathrm{MoS}_{2}$ ) compounds. The $\mathrm{MPS}_{3}$ phases are taken as layered $\mathrm{MS}_{2}$ crystals with one third of the $M$ sites substituted by $P-P$ pairs $\left(P_{2}\right)$, i.e.,
$\mathrm{M}_{2 / 3}\left(\mathrm{P}_{2}\right)_{1 / 3} \mathrm{~S}_{2}$. The sulfur atoms comprise the surfaces within individual lamella. From Figure 3a, an octahedrally coordinated configuration can be visualized in which the $2 / 3$ is filled by $\mathrm{M}^{\mathrm{II}}$ cations and the remaining $1 / 3$ is occupied by $\mathrm{P}-\mathrm{P}$ dimers. The $\mathrm{P}-\mathrm{P}$ dimers are covalently bonded to six sulfur atoms to form an ethane-like $\left(\mathrm{P}_{2} \mathrm{~S}_{6}\right)^{4-}$ unit, where each $\mathrm{P}$ atom is tetrahedrally coordinated with three $\mathrm{S}$ atoms. Meanwhile, a sulfur atom is coordinated with two $\mathrm{M}^{\mathrm{II}}$ sites and is covalently bonded to one $\mathrm{P}$ atom.

Generally, the overall structure of the individual lamella is similar across the $\mathrm{MPX}_{3}$ family members, but their stacking arrangements in bulk vary depending on the cations and anions constituting the crystals. Their symmetry and lamellar stacking are summarized in Table 1. There is an obvious distinction between the sulfides and selenides encountered in their symmetry and crystal structures. The widest symmetry of $\mathrm{M}^{\mathrm{II}} \mathrm{PS}_{3}$ is $\mathrm{C} 2 / \mathrm{m}$ with monoclinic crystal structure, but $\mathrm{HgPS}_{3}$ crystallizes into a space group of $P 1^{[37]}$ along with distorted tetrahedrally coordinated $\mathrm{Hg}$ ions. ${ }^{[30]}$ In the $\mathrm{C} 2 / \mathrm{m}$ space group, the structure is constructed from sulfur cubic close-packed arrays with the octahedral sites in each layer completely filled by $\mathrm{M}^{\mathrm{II}}$ and $\mathrm{P}-\mathrm{P}$ pairs at a $2 / 1$ ratio. One observes that the value of monoclinic angle $\beta$, varying from $106.97^{\circ}$ for $\mathrm{MgPS}_{3}$ to $107.35^{\circ}$ for $\mathrm{MnPS}_{3}$, is different based on $\mathrm{M}$ cations (Figure $3 \mathrm{~b}$ ). For an undistorted monoclinic cell, the value of $\beta$ is calculated to be $107.16^{\circ}$, suggesting that $\mathrm{CoPS}_{3}$ and $\mathrm{FePS}_{3}$ possess a perfect cell. ${ }^{[38]}$ All $\mathrm{M}^{\mathrm{II}} \mathrm{PX}_{3}$ materials with the $\mathrm{C} 2 / \mathrm{m}$ space show a layers stacking sequence of "AAA" (Table 1).

For $\mathrm{M}^{\mathrm{II} P \mathrm{Se}_{3}}$ (e.g., $\mathrm{CdPSe}_{3}, \mathrm{MgPSe}_{3}, \mathrm{FePSe}_{3}, \mathrm{ZnPSe}_{3}$ ), owing to the enlarged $\mathrm{P}-\mathrm{Se}$ bond distance and $\mathrm{Se}-\mathrm{P}-\mathrm{Se}$ bond angles, their space group has been reported as $R 3(-) h .{ }^{[39-41]}$ The distortions exhibited in the $\mathrm{PSe}_{3}$ groups on the bottom halves of one layer result in the lack of an inversion center for these $\mathrm{MPSe}_{3}$ crystals. Otherwise, $\mathrm{NiPSe}_{3}$ has the same symmetry as that of 


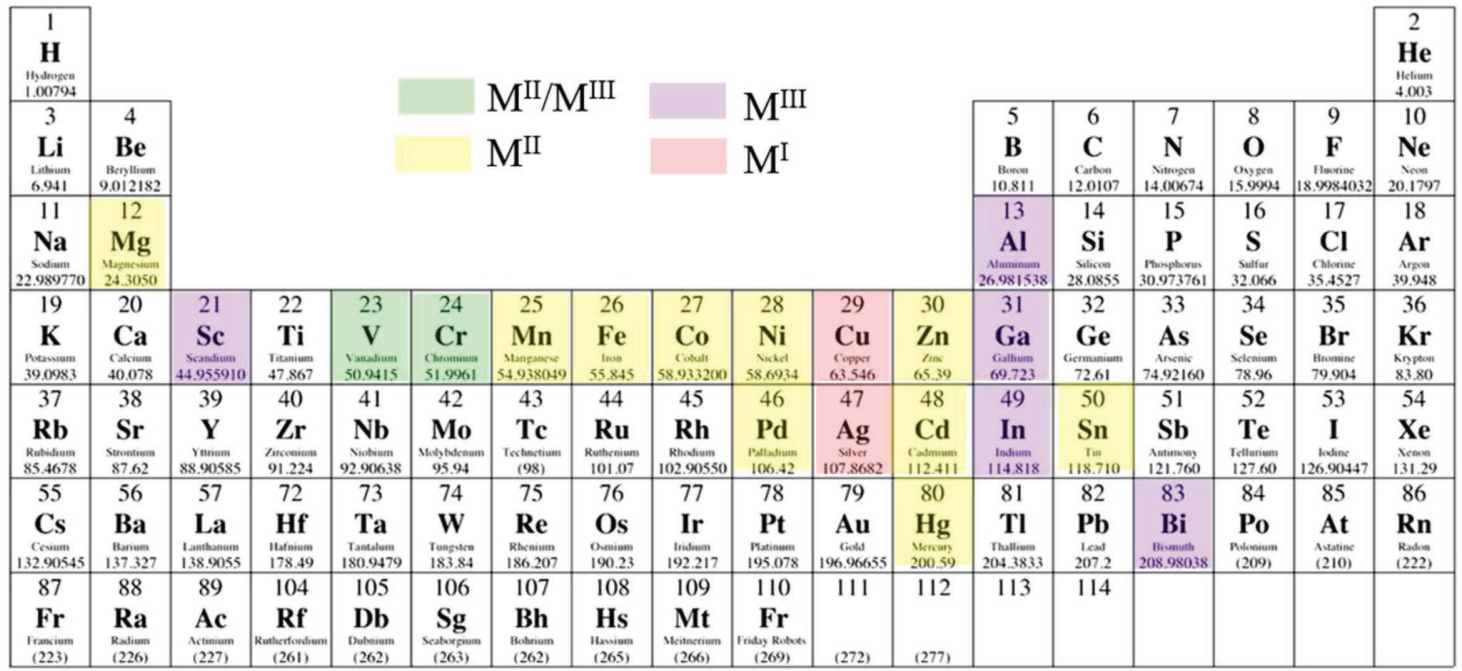

\begin{tabular}{|c|c|c|c|c|c|c|c|c|c|c|c|c|c|}
\hline $\begin{array}{l}58 \\
\mathrm{Ce}\end{array}$ & $\begin{array}{l}59 \\
\text { Pr }\end{array}$ & $\begin{array}{c}60 \\
\text { Nd }\end{array}$ & $\begin{array}{c}61 \\
\mathbf{P m}\end{array}$ & $\begin{array}{c}62 \\
\text { Sm }\end{array}$ & $\begin{array}{c}63 \\
\text { Eu }\end{array}$ & $\begin{array}{c}64 \\
\text { Gd }\end{array}$ & $\begin{array}{c}65 \\
\text { Tb }\end{array}$ & $\begin{array}{c}66 \\
\text { Dy }\end{array}$ & $\begin{array}{c}67 \\
\text { Ho }\end{array}$ & $\begin{array}{c}68 \\
\text { Er }\end{array}$ & $\begin{array}{c}69 \\
\text { Tm }\end{array}$ & $\begin{array}{c}70 \\
\text { Yb }\end{array}$ & $\begin{array}{l}71 \\
\text { Lu }\end{array}$ \\
\hline 140.116 & 140.90765 & 144.24 & $\begin{array}{l}\text { Promerther } \\
(145)\end{array}$ & $\begin{array}{l}\text { Samprumer } \\
150.36\end{array}$ & $\begin{array}{l}\text { Earpoum } \\
151.964\end{array}$ & $\begin{array}{l}\text { Gadtulu } \\
157.25\end{array}$ & $\begin{array}{l}\text { Trthiumum } \\
\text { S8.9253 }\end{array}$ & 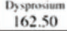 & 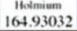 & $\begin{array}{l}\text { Ethem } \\
167.26\end{array}$ & $\begin{array}{l}108.934 \\
103421\end{array}$ & 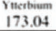 & $\begin{array}{l}\text { Litction } \\
174.967\end{array}$ \\
\hline 90 & 91 & 92 & 93 & 94 & 95 & 96 & 97 & 98 & 99 & 100 & 101 & 102 & 103 \\
\hline Th & $\mathbf{P a}$ & $\mathbf{U}$ & Np & $\mathbf{P u}$ & Am & $\mathrm{Cm}$ & Bk & Cf & Es & Fm & Md & No & $\mathbf{L r}$ \\
\hline 0 & & & 2377) & $(244$ & (243) & (247) & $(247)$ & 512 & (252) & (257) & $(25)$ & $\begin{array}{l}(259) \\
\text { (259) }\end{array}$ & $\begin{array}{l}\text { (262) } \\
(262)\end{array}$ \\
\hline
\end{tabular}

Figure 2. The metal atoms and their valence states constituted in $\mathrm{MPX}_{3}$ crystals in the periodic table of elements. Filled blocks represent elements for which the layered $\mathrm{MPX}_{3}$ structure has been reported to form.

$\mathrm{NiPS}_{3}(\mathrm{C} 2 / \mathrm{m})$. Based on the above descriptions, most selenides crystallize into monoclinic or trigonal crystallographic structures. The $\mathrm{M}^{\mathrm{II}} \mathrm{PSe}_{3}$ crystals with $R 3(-) \mathrm{h}$ symmetry demonstrate the lamellar stacking sequence of "ABC" (Table 1). $\mathrm{Hg}_{2} \mathrm{P}_{2} \mathrm{Se}_{6}$ adopts a unique structure with tilting of $\mathrm{P}-\mathrm{P}$ dimers, distorting the octahedral cages between the $\mathrm{P}_{2} \mathrm{Se}_{6}$ units, and its stacking sequence is "ABAB."
For layered $\mathrm{MPX}_{3}$ crystals, their interlayer distances (d, i.e., distance between two layers) vary from 6.3 to $6.7 \AA$ (Figure 3c). The ordering of $d$, namely, $d_{\mathrm{MnPS} 3}>d_{\mathrm{FePS} 3}>d_{\mathrm{NiPS} 3}$, is same as that of the $\mathrm{M}$ radius $(r)\left(r_{\mathrm{Mn}}>r_{\mathrm{Fe}}>r_{\mathrm{Ni}}\right)$. Additionally, selenides possess a larger interlayer distance than that of the corresponding sulfides, like $d_{\mathrm{MnPSe} 3}>d_{\mathrm{MnPS} 3}$. a)
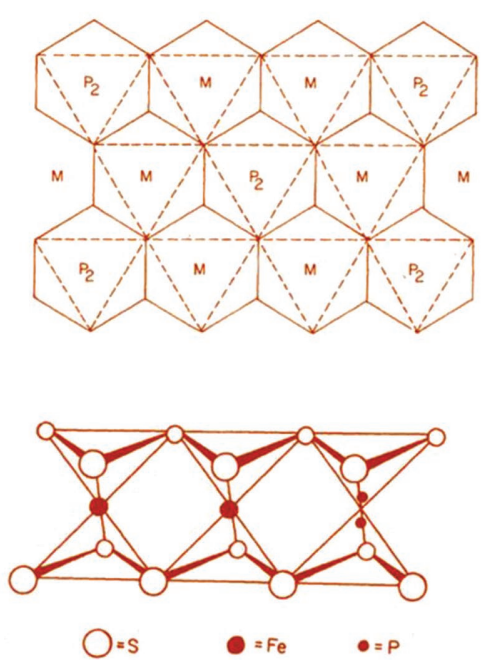

b)

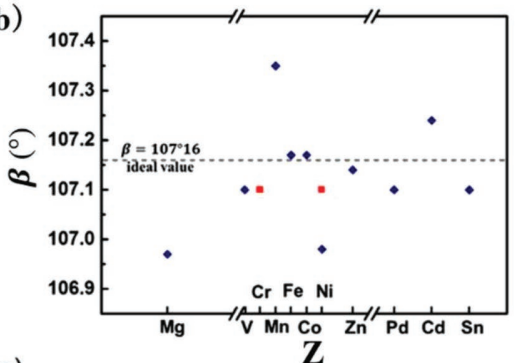

c)

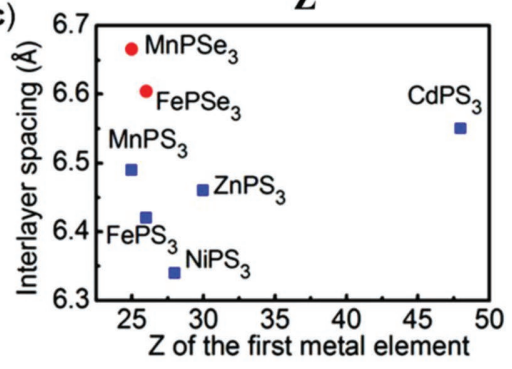

Figure 3. a) Structure of $\mathrm{FePS}_{3}$, showing the close structural coordination around the $\mathrm{Fe}^{2+}$. Reproduced with permission. ${ }^{[51]}$ Copyright 2018, American Physical Society. b) $\mathrm{MPS}_{3}$ monoclinic cell $\beta$ value variation respective to the $\mathrm{M}^{2+}$ cation. $\mathrm{MPS}_{3}$, dark blue " "; MPSe 3 , red " $\square$ ". c) Interlayer spacings of $\mathrm{MPS}_{3}$ and $\mathrm{MPSe}_{3}$. Reproduced with permission. ${ }^{[20]}$ Copyright 2016, American Chemical Society.

\subsubsection{Polymetallic $\mathrm{MPX}_{3}$ Compounds}

The "M" atoms in $\mathrm{MPX}_{3}$ crystals could be substituted by other metal atoms, including homocharge and heterocharge substitution. If the size of $\mathrm{M}_{1}{ }^{\mathrm{II}}$ and $\mathrm{M}_{2}{ }^{\mathrm{II}}$ cations is almost the same, the homocharge substitution will be realized easily. For example, a series of $\mathrm{Zn}_{1-x} \mathrm{Fe}_{x} \mathrm{PS}_{3}, \mathrm{Zn}_{1-x} \mathrm{Ni}_{x} \mathrm{PS}_{3}(0 \leq x \leq 1),{ }^{[29]}$ and $\mathrm{Cd}_{x} \mathrm{Fe}_{1-x} \mathrm{P}_{2} \mathrm{~S}_{6}(0 \leq x \leq 1)^{[42]}$ has been obtained. Significantly, the $\mathrm{MPX}_{3}$ structural type also shows that one $\mathrm{M}^{\mathrm{II}}$ could be replaced by a couple of heterocharge metal atoms, namely, $1 / 2 \mathrm{M}_{\mathrm{I}}$ and $1 / 2 \mathrm{M}_{\mathrm{III}} \cdot \mathrm{M}_{\mathrm{I}}$ includes $\mathrm{Ag}^{+}, \mathrm{Cu}^{+}$, and $\mathrm{M}_{\mathrm{III}}$ contains $\mathrm{In}^{3+}$, $\mathrm{V}^{3+}, \mathrm{Sc}^{3+}$, and $\mathrm{Cr}^{3+}$, etc. The structure of the mixed-cation $\mathrm{M}^{\mathrm{I}} \mathrm{M}^{\mathrm{III}}\left[\mathrm{P}_{2} \mathrm{~S}_{6}\right]^{4-}$ compounds is easily tuned. In the 1980s, the synthesized $\mathrm{AgInP}_{2} \mathrm{~S}_{6}{ }^{[43]}$ and $\mathrm{AgScP}_{2} \mathrm{~S}_{6}{ }^{[42]}$ crystals were identified as trigonal $(P 1(-) 3 \mathrm{c})$ and centrosymmetric structures at room temperature. Of special note is that the $\mathrm{Cu}^{+}$cations based $\mathrm{CuM}^{\mathrm{III}} \mathrm{P}_{2} \mathrm{~S}_{6}$ compounds are unique. For instance, there are three possible $\mathrm{Cu}^{+}$ 
Table 1. Structural information for the 2D layered $M^{\prime \prime} \mathrm{PX}_{3}$ crystals.

\begin{tabular}{|c|c|c|c|c|c|c|c|c|c|c|}
\hline & \multirow[t]{2}{*}{ Components } & \multicolumn{4}{|c|}{$\mathrm{S}$ based } & \multirow[t]{2}{*}{ Components } & \multicolumn{4}{|c|}{ Se based } \\
\hline & & Crystal structures & Space group & Stacking ${ }^{\text {a) }}$ & Ref. & & Crystal structures & Space group & Stacking ${ }^{\text {a) }}$ & Ref. \\
\hline \multirow[t]{12}{*}{$\overline{M^{\prime \prime}}$} & $\mathrm{ZnPS}_{3}$ & Monoclinic & $\mathrm{C} 2 / \mathrm{m}$ & AAA & [117] & $\mathrm{ZnPSe}_{3}$ & Rhombohedral & $\mathrm{R} \overline{3}$ & $A B C$ & [89] \\
\hline & $\mathrm{CdPS}_{3}$ & Monoclinic & $\mathrm{C} 2 / \mathrm{m}$ & AAA & {$[18]$} & $\mathrm{CdPSe}_{3}$ & Rhombohedral & $R \overline{3}$ & $A B C$ & [41] \\
\hline & $\mathrm{MnPS}_{3}$ & Monoclinic & $\mathrm{C} 2 / \mathrm{m}$ & AAA & [18] & $\mathrm{MnPSe}_{3}$ & Rhombohedral & $\mathrm{R} \overline{3}$ & $A B C$ & [39] \\
\hline & $\mathrm{FePS}_{3}$ & Monoclinic & $\mathrm{C} 2 / \mathrm{m}$ & AAA & [18] & $\mathrm{FePSe}_{3}$ & Rhombohedral & $\mathrm{R} \overline{3}$ & $A B C$ & [39] \\
\hline & $\mathrm{NiPS}_{3}$ & Monoclinic & $\mathrm{C} 2 / \mathrm{m}$ & AAA & {$[41]$} & $\mathrm{NiPSe}_{3}$ & Monoclinic & $\mathrm{C} 2 / \mathrm{m}$ & AAA & [41] \\
\hline & $\mathrm{VPS}_{3}$ & Monoclinic & $\mathrm{C} 2 / \mathrm{m}$ & AAA & [89] & $\mathrm{VPSe}_{3}$ & - & - & - & - \\
\hline & $\mathrm{CrPS}_{3}$ & - & - & - & - & $\mathrm{CrPSe}_{3}$ & Monoclinic & $\mathrm{C} 2 / \mathrm{m}$ & AAA & [41] \\
\hline & $\mathrm{CoPS}_{3}$ & Monoclinic & $\mathrm{C} 2 / \mathrm{m}$ & AAA & [89] & $\mathrm{CoPSe}_{3}$ & - & - & - & - \\
\hline & $\mathrm{MgPS}_{3}$ & Monoclinic & $\mathrm{C} 2 / \mathrm{m}$ & AAA & [89] & $\mathrm{MgPSe}_{3}$ & Rhombohedral & $\mathrm{R} \overline{3}$ & $A B C$ & [89] \\
\hline & $\mathrm{PdPS}_{3}$ & Monoclinic & $\mathrm{C} 2 / \mathrm{m}$ & AAA & [89] & $\mathrm{PdPSe}_{3}$ & - & - & - & - \\
\hline & $\mathrm{SnPS}_{3}$ & Monoclinic & $\mathrm{C} 2 / \mathrm{m}$ & AAA & [118] & $\mathrm{SnPSe}_{3}$ & - & - & - & - \\
\hline & $\mathrm{HgPS}_{3}$ & Triclinic & $\mathrm{P} \overline{1}$ & AAA & [37] & $\mathrm{HgPSe}_{3}$ & Monoclinic & $\mathrm{C} 2 / \mathrm{c}$ & ABAB & [37] \\
\hline
\end{tabular}

a) The $c$ stacking direction; - denotes data not mentioned in the publications.

sites in $\mathrm{CuInP}_{2} \mathrm{~S}_{6}$ and the temperature could affect the occupancy. ${ }^{[4]}$ The $\mathrm{Cu}$ atom hopping motions have indirectly been proved with single-crystal X-ray diffraction. When the temperature is above $315 \mathrm{~K}$, the $\mathrm{Cu}^{\mathrm{I}}$ occupancy splits evenly into the upper and lower sites. Otherwise, the upper site is preferentially filled at $T<315 \mathrm{~K}$. Compared to the midplane of the lamella, the $\mathrm{Cu}^{\mathrm{I}}$ cations were shifted upward by $1.58 \AA$, while the In ${ }^{\text {III }}$ shifted in the opposite direction by $0.2 \AA$. Two polar sublattices, which are formed by partial compensation of the two different cations, produce ferrielectric materials due to the huge spontaneous polarization in the stacking direction. However, in $\mathrm{CuCrP}_{2} \mathrm{~S}_{6}$ and $\mathrm{CuVP}_{2} \mathrm{~S}_{6}$ crystals, the shifts and transitions are different. ${ }^{[4,46]}$ Of the $\mathrm{M}^{\mathrm{I}} \mathrm{M}^{\mathrm{III}} \mathrm{P}_{2} \mathrm{~S}_{6}$ crystals, most of them exhibit an $\mathrm{ABAB}$ stacking sequence with various space groups. Notably, $\mathrm{AgVP}_{2} \mathrm{~S}_{6}$ and $\mathrm{AgCrP}_{2} \mathrm{~S}_{6}$ compounds have buckled crystal structures, likely resulting from the much larger $\mathrm{Ag}^{+}(1.15 \AA)$ ions than the $\mathrm{Cr}^{3+}(0.62 \AA)$ and $\mathrm{V}^{3+}$ $(0.64 \AA) \cdot{ }^{[47]}$ Thus, Ag atoms do not vary their locations within the sulfur-defined octahedral but they are limited to the center of the layers. Similarly, $\mathrm{M}^{\mathrm{I}} \mathrm{M}^{\mathrm{III}} \mathrm{P}_{2} \mathrm{Se}_{6}$ shows the structures that are depicted in Figure 1. The compounds of $\mathrm{CuAlP}_{2} \mathrm{Se}_{6}$, $\mathrm{AgAlP}_{2} \mathrm{Se}_{6}$, and $\mathrm{AgCrP}_{2} \mathrm{Se}_{6}$ are reported to exhibit random cation ordering, which does not exist in the other alloyed compounds. Their crystal structure and stacking sequences are shown in Table 2. Exceptionally, the existence of three temperature phases (Figure 4) occur in $\mathrm{CuBiP}_{2} \mathrm{Se}_{6} \cdot{ }^{[48]}$ Their stacking sequences are ABAB (298 K, P3(-)c), ABCDEF (173 K, R3(-)h), and ABCDEFGHIJKL (97 K, R3(-h)), respectively, suggesting the extended stacking of six to twelve layers before the observed translational symmetry at low temperature.

\subsection{Unique Properties of $\mathrm{MPX}_{3}$ Compounds}

Owing to the special crystal structure of $\mathrm{MPX}_{3}$ compounds, many important features, including electrical, magnetic, and optical properties, are demonstrated. ${ }^{[30]}$ On the one hand, the number of electrons in the outmost shell or $d$ orbital of the metal elements constituted in $\mathrm{MPX}_{3}$ contributes to their unique properties. On the other hand, the $\left[\mathrm{P}_{2} \mathrm{X}_{6}\right]^{4-}$ provides a weak ligand field, resulting in a high spin state of the metal atoms. ${ }^{[49,50]}$ Accordingly, a plethora of results about the magnetism of these materials are published from the perspective of experiments and calculations. The electronic configuration varies corresponding to the different cations. These features will be discussed in the following section.

\subsubsection{Electronic Structure}

The fascinating electronic band model of $\mathrm{MPX}_{3}$ layered compounds aroused extensive attention in 1980s. ${ }^{[51]}$ The electronic energy-band scheme of the $\mathrm{MPX}_{3}$ family, e.g., $\mathrm{MnPS}_{3}, \mathrm{FePS}_{3}$, and $\mathrm{NiPS}_{3}$, has been studied through the vacuum ultraviolet reflectivity spectra and X-ray absorption spectra. In terms of the molecular orbital and crystal-field theories, Khumalo and Hughes ${ }^{[51]}$ found that high-spin divalent $M$ ions are octahedrally coordinated, and the octahedral ligand of $\left[\mathrm{P}_{2} \mathrm{~S}_{6}\right]$ field splits the $d^{5}\left(\mathrm{Mn}^{2+}\right)$ orbitals into $\mathrm{t}_{2 \mathrm{~g}}{ }^{3}$ and $\mathrm{e}_{\mathrm{g}}{ }^{2}, d^{6}\left(\mathrm{Fe}^{2+}\right)$ orbitals into $\mathrm{t}_{2 \mathrm{~g}}{ }^{4}$ and $\mathrm{e}_{\mathrm{g}}{ }^{2}$, and $d^{8}\left(\mathrm{Ni}^{2+}\right)$ orbitals into $\mathrm{t}_{2 \mathrm{~g}}{ }^{6}$ and $\mathrm{e}_{\mathrm{g}}{ }^{2}$. The energy level scheme shown in Figure $5 \mathrm{a}^{[50]}$ demonstrates the energy level of $\left[\mathrm{P}_{2} \mathrm{~S}_{6}\right]^{4-}$ clusters and the localized $3 d$ levels of $\mathrm{M}^{\mathrm{II}}$ ions. Irrespective of the metal $3 d$ levels, the valence band and conduction band edges consist of the bonding and the antibonding levels derived from the $\mathrm{P}-\mathrm{P}$ bonds. In the ionic band-model scheme with divalent metal cations, the $\mathrm{P}-\mathrm{P}$ pair possesses a valence of +8 , suggesting that one electron in $\mathrm{P}$ atom contributed to the formation of chemical bond between two P atoms. ${ }^{[51]}$ This electronic structure calculated from the ionic extreme of the Wilson-Yoffe band model was adopted to explain the unusual optical absorption spectra, which reveals that the $\mathrm{MPS}_{3}$ compounds exhibit semiconducting 
Table 2. Structural information for the $2 \mathrm{D}$ layered $\mathrm{M}^{\prime} \mathrm{M}^{\prime \prime \prime} \mathrm{PX}{ }_{3}$ crystals.

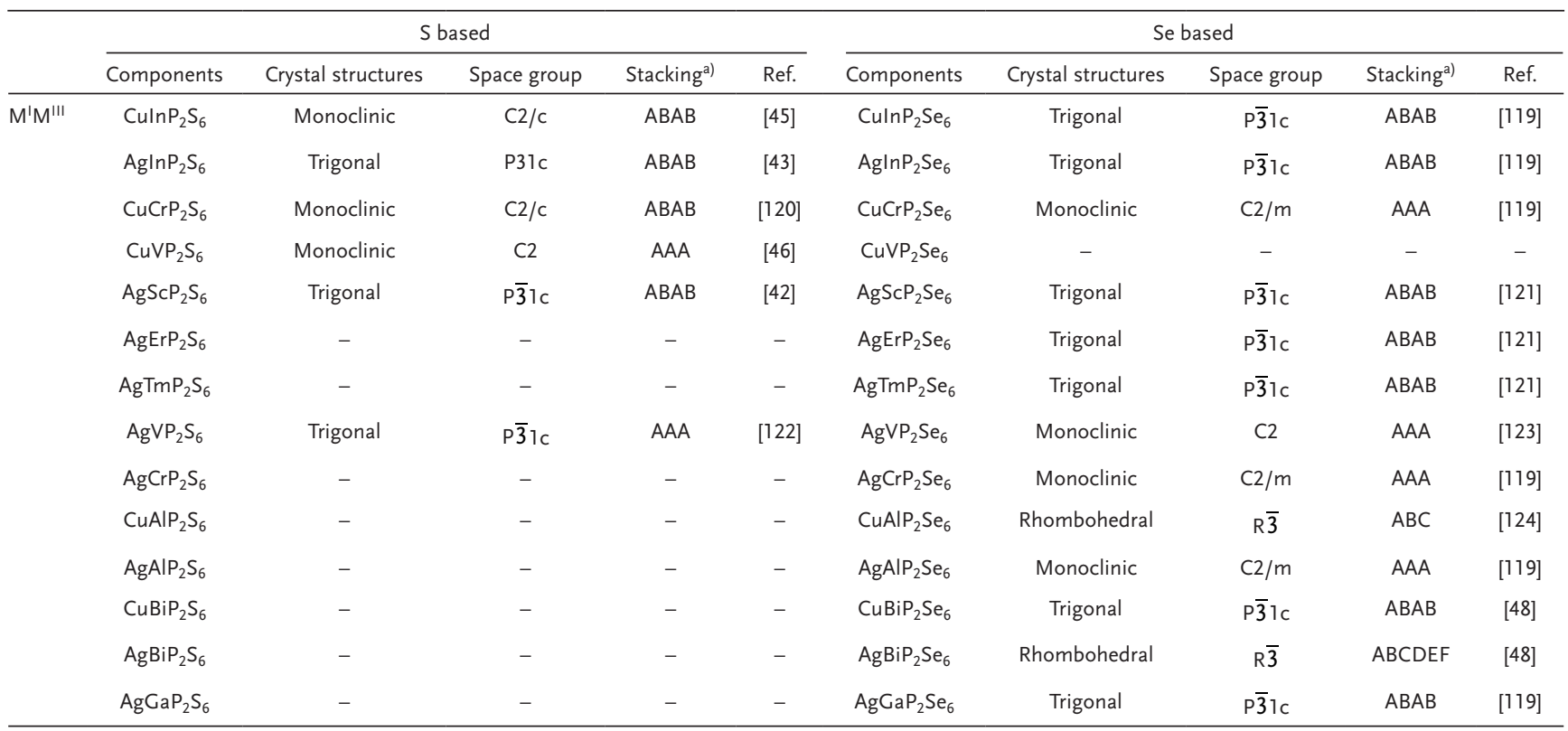

a) The $c$ stacking direction; - denotes data not mentioned in the publications.

behavior. Further refined Wilson-Yoffe model and extended Huckel model ${ }^{[52]}$ were utilized to estimate a band structure. These $\mathrm{M}^{\mathrm{II}} \mathrm{PS}_{3}$ ( $\mathrm{M}^{\mathrm{II}}$ : first raw series transition metals) compounds possess similar electronic band structures. Some of the known semiconducting $\mathrm{MPX}_{3}$ compounds have been surveyed and collected in Table 3 . The value of their bandgaps ranges from $1.3 \mathrm{eV}$ for $\mathrm{FePS}_{3}$ to $3.5 \mathrm{eV}$ for $\mathrm{CdPS}_{3} \cdot{ }^{[49]} \mathrm{Com}$ pared with the bandgaps of sulfides, those of selenides are smaller due to the relative electronegativity of S and Se atoms. It can be seen that the value of the bandgap is closely related to the metal cations present in the compound. Additionally, in 1996, Zhukov et al. ${ }^{[53]}$ calculated and discussed the relation between the electronic structure and the spin polarization in metal ions. The linear muffin-tin method with the atomic sphere approximation was utilized. The $3 d$ regions of the density of state (DOS) of $\mathrm{MPS}_{3}$, including $\mathrm{MnPS}_{3}, \mathrm{FePS}_{3}$, and $\mathrm{NiPS}_{3}$, are displayed in Figure 5b, demonstrating the relative energies of $t_{2 g}$ and $e_{g}$ bands and their occupations with the shaded areas. The spin-up states are more stable with lower energy than the spin-down ones, indicating they are at a high-spin configuration, consistent with the results based on experiments. ${ }^{[18,50]}$

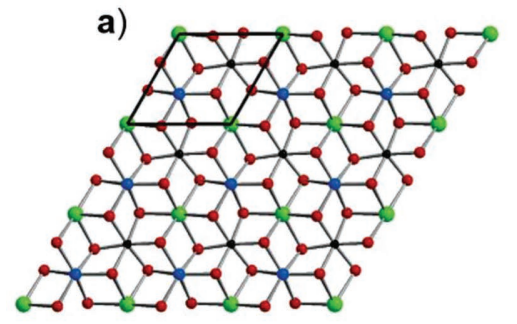

b)

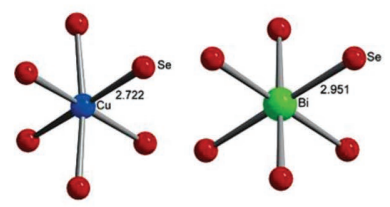

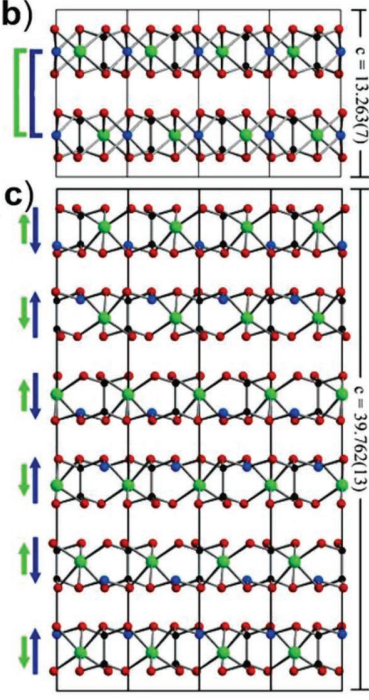

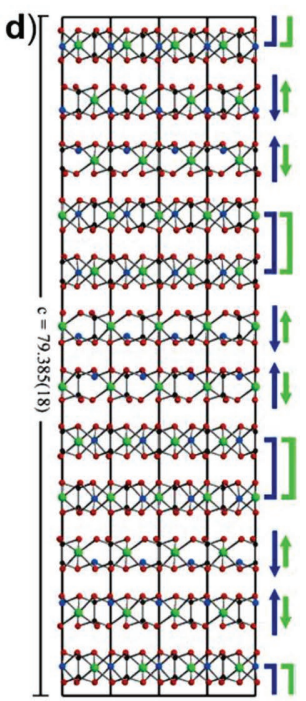

Figure 4. a) View of a single $\mathrm{CuBiP}_{2} \mathrm{Se}_{6}$ layer down the $c$-axis [(001) direction] showing the ordered arrangement of the metal ions (top) and the immediate coordination environment of $\mathrm{Cu}+$ and $\mathrm{Bi}^{3+}$ in $\mathrm{CuBiP}_{2} \mathrm{Se}_{6}$ at room temperature. b) $\left.298 \mathrm{~K}, \mathrm{c}\right) 173 \mathrm{~K}$, and d) $97 \mathrm{~K}$ structures of $\mathrm{CuBiP}_{2} \mathrm{Se}_{6}$ viewed down the ${ }^{[110]}$ direction. Reproduced with permission. ${ }^{[48]}$ Copyright 2005, American Chemical Society. 

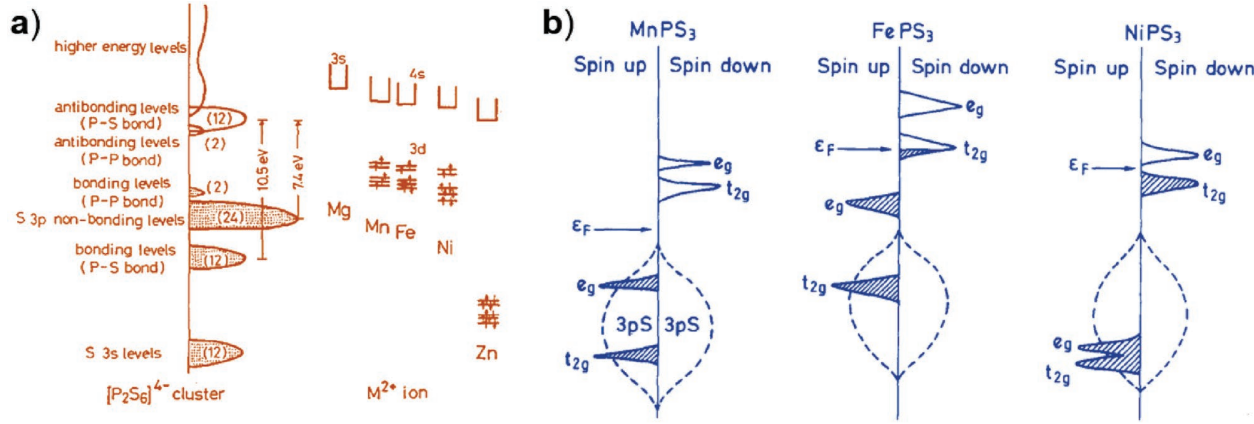

Figure 5. a) Energy level scheme of $\mathrm{MPS}_{3}$. It is constructed from the energy levels of $\left[\mathrm{P}_{2} \mathrm{~S}_{6}\right]^{4-}$ clusters and localized divalent metal ions based on the absorption spectra. Reproduced with permission. ${ }^{[50]}$ Copyright 1986, Elsevier B.V. b) $3 d$ region of the DOS of MPS ${ }_{3}$ showing the relative energies of the spin-polarized $\mathrm{t}_{2 \mathrm{~g}}$ and $\mathrm{e}_{\mathrm{g}}$ bands and their occupations. Reproduced with permission. ${ }^{[53]}$ Copyright 1996, Elsevier Ltd.

From the perspective of band theory, the orbital projected partial DOS of single-layer $\mathrm{MnPS}_{3}, \mathrm{FePS}_{3}$, and $\mathrm{NiPS}_{3}$ are demonstrated in Figure 6 ${ }^{[54]}$ revealing that the $s$ and $p$ orbitals of the $\mathrm{P}$ atoms mainly contribute to the conduction-band edges, while the top of valence band is significantly contributed by the chalcogen's atomic orbitals. Notably, considering the specific

Table 3. Reported calculated and experimental bandgaps for various $\mathrm{MPX}_{3}$ compounds.

\begin{tabular}{|c|c|c|c|c|c|}
\hline \multirow[t]{3}{*}{ Components } & \multicolumn{3}{|c|}{ Bandgaps $^{[20]}$} & \multirow{3}{*}{ Experimental } & \multirow{3}{*}{$\begin{array}{c}\text { Type } \\
\text { Indirect/Direct }\end{array}$} \\
\hline & \multicolumn{3}{|c|}{ Calculated } & & \\
\hline & $E_{g}$ & $\mathrm{CBM}$ & VBM & & \\
\hline $\mathrm{ZnPS}_{3}$ & $3.30^{\mathrm{ML}}(\mathrm{HSEO6})^{[93]}$ & -3.34 & -6.64 & $3.5^{\mathrm{B}[20]}$ & Indirect \\
\hline $\mathrm{CdPS}_{3}$ & $3.03^{\mathrm{ML}}(\mathrm{HSEO} 6)^{[93]}$ & -3.70 & -6.73 & $3.4^{\mathrm{B}[20]}$ & Indirect \\
\hline $\mathrm{MnPS}_{3}$ & $3.05^{\mathrm{ML}}(\mathrm{HSE06})^{[54]}$ & - & - & $3.0^{\mathrm{B}[49]}$ & Direct \\
\hline $\mathrm{FePS}_{3}$ & $2.54^{\mathrm{ML}}(\mathrm{HSEO} 6)^{[21]}$ & - & - & $1.5^{\mathrm{B}[49]}$ & Indirect \\
\hline $\mathrm{NiPS}_{3}$ & $3.01^{\mathrm{ML}}(\mathrm{HSEO6})^{[21]}$ & - & - & $1.6^{\mathrm{B}[49]}$ & Indirect \\
\hline $\mathrm{VPS}_{3}$ & $2.50^{\mathrm{ML}}(\mathrm{HSEO} 6)^{[54]}$ & - & - & - & Indirect \\
\hline $\mathrm{CuPS}_{3}$ & $1.76^{\mathrm{ML}}(\mathrm{HSEO} 6)^{[54]}$ & - & - & - & Indirect \\
\hline $\mathrm{MgPS}_{3}$ & $3.94^{\mathrm{ML}}(\mathrm{HSEO6})^{[93]}$ & -3.07 & -7.01 & - & Direct \\
\hline Culn $\mathrm{P}_{2} \mathrm{~S}_{6}$ & - & - & - & $2.9^{\mathrm{B}[125]}$ & Direct \\
\hline $\mathrm{AgScP}{ }_{2} \mathrm{~S}_{6}$ & $3.16^{\mathrm{ML}}(\mathrm{HSEO} 06)^{[93]}$ & -3.55 & -6.71 & - & Direct \\
\hline $\mathrm{AgBiP}_{2} \mathrm{~S}_{6}$ & - & - & - & $1.7^{\mathrm{B}[125]}$ & - \\
\hline $\mathrm{Ag} \ln \mathrm{P}_{2} \mathrm{~S}_{6}$ & $2.41^{\mathrm{ML}}(\mathrm{HSEO} 06)^{[93]}$ & -3.65 & -6.06 & - & Indirect \\
\hline $\mathrm{CdPSe}_{3}$ & $3.03^{\mathrm{ML}}(\mathrm{HSEO} 06)^{[93]}$ & -3.70 & -6.73 & $2.29^{\mathrm{B}[126]}$ & Indirect \\
\hline $\mathrm{ZnPSe}_{3}$ & $2.32^{\mathrm{ML}}(\mathrm{HSEO} 06)^{[93]}$ & -3.71 & -6.02 & - & Indirect \\
\hline $\mathrm{FePSe}_{3}$ & $1.48^{\mathrm{ML}}(\mathrm{HSEO} 06)^{[54]}$ & - & - & $1.3^{B[49]}$ & Indirect \\
\hline $\mathrm{MnPSe}_{3}$ & $2.24^{\mathrm{ML}}(\mathrm{HSE} 06)^{[54]}$ & - & - & $2.5^{\left[\mathrm{B}^{[49]}\right.}$ & Direct \\
\hline $\mathrm{VPSe}_{3}$ & $2.10^{\mathrm{ML}}(\mathrm{HSEO6})^{[54]}$ & - & - & - & Indirect \\
\hline $\mathrm{NiPSe}_{3}$ & $2.22^{\mathrm{ML}}(\mathrm{HSE06})^{[2]]}$ & - & - & - & Indirect \\
\hline $\mathrm{CuPSe}_{3}$ & $0.56^{\mathrm{ML}}(\mathrm{HSE} 06)^{[54]}$ & - & - & - & Indirect \\
\hline $\mathrm{MgPSe}_{3}$ & $2.97^{\mathrm{ML}}(\mathrm{HSEO6})^{[93]}$ & -3.38 & -6.35 & - & Direct \\
\hline $\mathrm{CuBiP}_{2} \mathrm{Se}_{6}$ & - & - & - & $1.2^{\mathrm{B}[48]}$ & Indirect \\
\hline $\mathrm{AgBiP}_{2} \mathrm{Se}_{6}$ & - & - & - & $1.4^{\mathrm{B}[48]}$ & - \\
\hline $\mathrm{AgScP}_{2} \mathrm{Se}_{6}$ & $2.66^{\mathrm{ML}}(\mathrm{HSEO} 06)^{[93]}$ & -3.79 & -6.45 & $1.55^{8[121]}$ & Direct \\
\hline $\mathrm{Ag} \ln \mathrm{P}_{2} \mathrm{Se}_{6}$ & $1.77^{\mathrm{ML}}(\mathrm{HSE06})^{[93]}$ & -4.03 & -5.80 & - & Indirect \\
\hline
\end{tabular}

$M L$ denotes the "monolayer" of $\mathrm{MPX}_{3}$. B denotes the "bulk crystal" of $\mathrm{MPX}_{3}$. composition and magnetic configuration of these $\mathrm{MPX}_{3}$ materials, the edges of valence band and conduction band are dominated by metal, phosphorus, or chalcogen atoms. The crystal field splitting of the $d$-orbitals and the filling fractions affect their electronic structures near the Fermi level. ${ }^{[54]}$ The ab initio calculations conducted by Chittari et al. ${ }^{[54]}$ predict that most bandgaps of the anti-ferromagnetic $\mathrm{MPX}_{3}$ crystals decrease with the increase of the atomic number of the chalcogen. Thus, halfmetallic structure with ferromagnetic states or nonmagnetic metallic states is properly obtained in some cases.

Regarding other metals, e.g., $\mathrm{Mg}$ and $\mathrm{Zn}$ based $\mathrm{MPX}_{3}$ structures, their electronic DOS and orbital contribution are somehow different. $\mathrm{MgPS}_{3}$ contains no $d$ electrons. Its valence band consists primarily of $\mathrm{S}$ and $\mathrm{Mg}$ states, and $\mathrm{S}$ and $\mathrm{P}$ states dominate its conduction band. While $\mathrm{ZnPS}_{3}$, whose $d$ orbital shell is fully filled, shows the sharply localized $3 d$-levels below the top of the valence band. ${ }^{[55]}$ Recently, the band diagram for $\mathrm{CuInP}_{2} \mathrm{~S}_{6}$ was calculated by using density functional theory (DFT)-based ab initio theory to simulate the Jahn-Teller effect, which would make a contribution to the ferroelectric ordering of $\mathrm{CuInP}_{2} \mathrm{~S}_{6} \cdot{ }^{[56]}$

\subsubsection{Optical Properties}

The research on the optical properties of layered $\mathrm{MPX}_{3}$ crystals started in the early 1880s, ${ }^{[57]}$ Piacentini et al. ${ }^{[57]}$ collected the optical absorption spectra in the energy range $0-5.4 \mathrm{eV}$ for $\mathrm{NiPS}_{3}$ at different temperatures $(300,78$, and $4.2 \mathrm{~K})$. The features observed in the spectrum were interpreted to explain the transitions within the different $\mathrm{Ni}^{2+} 3 d$ levels and transitions between the $\mathrm{S} 3 p_{x} p_{y}$ valence states and the $\mathrm{Ni}^{2+} 3 d$ empty states (e $e_{g}$ orbital) (Figure 5a). Furthermore, the study about the Raman spectra of 

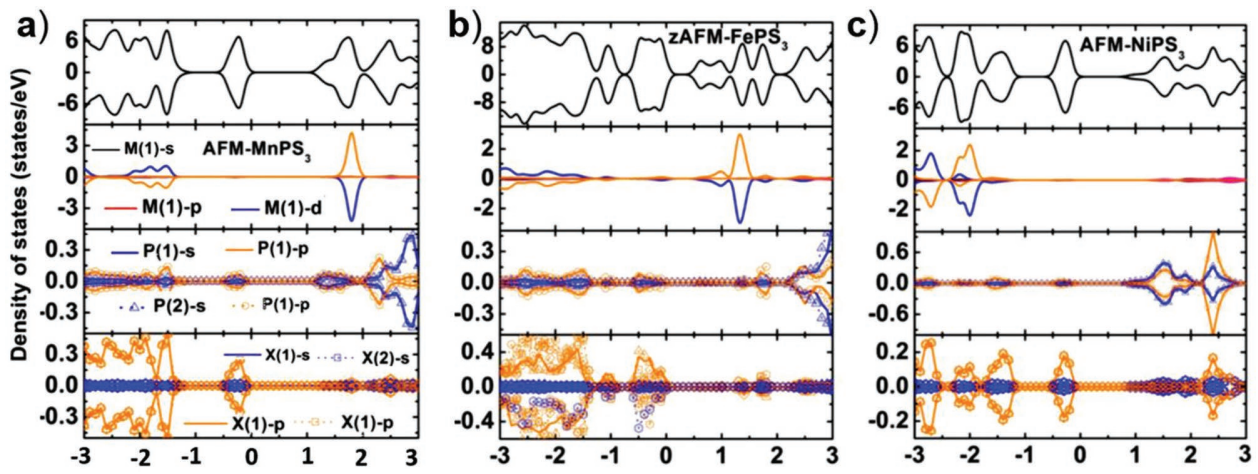

Figure 6. Orbitally projected partial density of the states (PDOS) of a) $\mathrm{MnPS}_{3}$, b) $\mathrm{FePS}_{3}$, and c) $\mathrm{NiPS}_{3}$ calculated for self-consistently converged groundstate magnetic configurations. Reproduced with permission. ${ }^{[54]}$ Copyright 2016, American Physical Society.

various layered 2D materials is a hot topic. As a popular characterization tool, the features present in the Raman spectra are helpful to estimate the thickness of nanosheets, such as graphene and TMDs. ${ }^{[58]} \mathrm{Du}$ et al. ${ }^{[20]}$ studied the Raman spectra of $\mathrm{FePS}_{3}$ nanosheets with different thicknesses, revealing the strong interlayer vibrations in three-layered $\mathrm{FePS}_{3}$ and stable structure of monolayer $\mathrm{FePS}_{3}$ under ambient conditions (Figure 7a). The comprehensive investigation ${ }^{[59]}$ of the layernumber and temperature dependent Raman spectra shows a magnetic persistence in $\mathrm{FePS}_{3}$ nanosheets with different layers, including single layered $\mathrm{FePS}_{3}$ nanosheet. But the Neel temperature of the anti-ferromagnetic $\mathrm{FePS}_{3}$ sample reduces from $117 \mathrm{~K}$ in bulk to $104 \mathrm{~K}$ in the monolayer. Some more works $^{[60,61]}$ on the Raman spectroscopic study reveal a similar trend for $\mathrm{NiPS}_{3}$ nanosheet which also exhibits a substantially varying feature depending on the number of layers. A remarkable linear relation is found between the Raman intensity of the peak for $\mathrm{A}_{\mathrm{g}}$ mode of $\mathrm{NiPS}_{3}$ on a sapphire substrate and the thickness in Figure $7 \mathrm{~b}, \mathrm{c},{ }^{[61]}$ which provides a simple way to estimate their thickness.

\subsubsection{Magnetism}

Recently, the discovery and characterization of magnetism of novel 2D crystals have been a major research direction. ${ }^{[18,62]}$ Ultimately, the magnetic functionality of these 2D materials is employed for fabricating spintronic or magnetoelectronic applications. ${ }^{[63]} \mathrm{MPX}_{3}$ members, being an important family of layered 2D materials, have potential application in low-dimensional magnetic and spintronic devices. ${ }^{[28,64,65]}$ Until now, experimental studies and theoretical calculations of the magnetic properties of $\mathrm{MPX}_{3}$ have been widely performed. The experimental studies on magnetic properties were mainly focused on the $\mathrm{MPX}_{3}$ with bulk crystals, ${ }^{[39,64,66-68]}$ while the theoretical calculations of the spin-state were concentrated on single-layered $\mathrm{MPX}_{3}$ nanosheets. ${ }^{[21,54]}$ There are three types (Figure $8 \mathrm{a}$ ) of magnetic moments-distributions that were proposed for the $\mathrm{MPX}_{3}$ crystals $^{[29]}$ in $1900 \mathrm{~s}$. In $\mathrm{NiPS}_{3}$ and $\mathrm{CoPS}_{3}$ monolayers, double parallel ferromagnetic chains are anti-ferromagnetically coupled (type I) with each other. For $\mathrm{MnPS}_{3}$ layers, a magnetic ion anti-ferromagnetically coupled with the three nearest neighbors
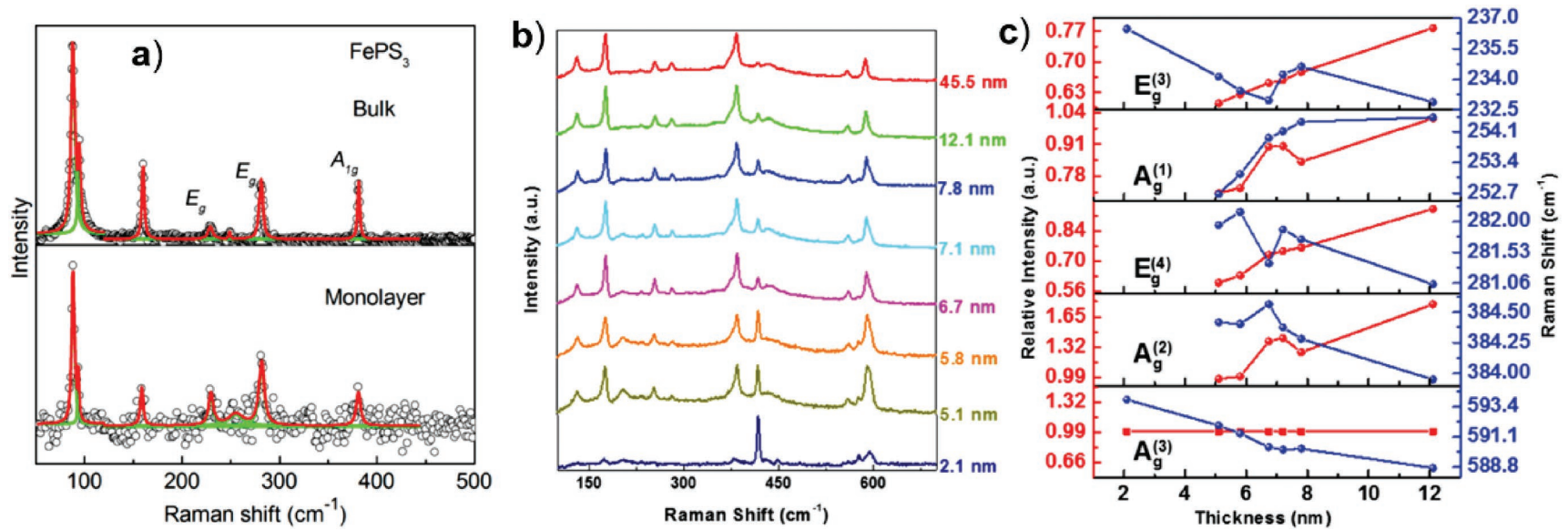

Figure 7. a) Raman spectra of bulk and monolayer $\mathrm{FePS}_{3}$ obtained with a $532 \mathrm{~nm}$ laser at $\approx 77 \mathrm{~K}$. Reproduced with permission. ${ }^{[20]}$ Copyright 2015 , American Chemical Society. b) Raman spectra of nanosheets on sapphire substrate with varying thickness from 2.1 to $45.5 \mathrm{~nm}$. c) The thickness dependence of relative intensity (red curve) and Raman shift (blue curve) of selected modes in (b). Reproduced with permission. ${ }^{61]}$ Copyright, 2017 , Wiley-VCH. 
a)
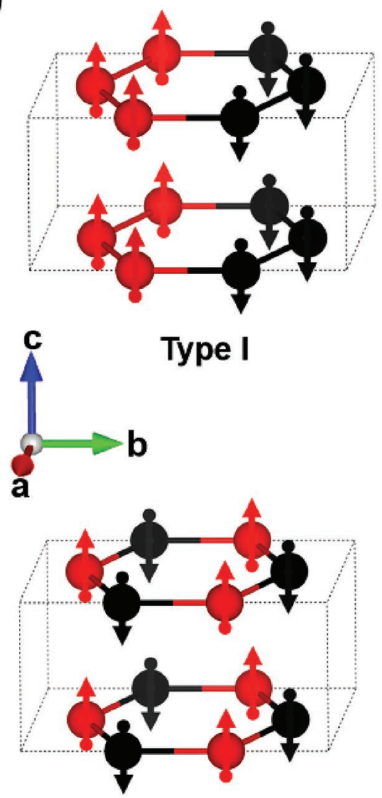

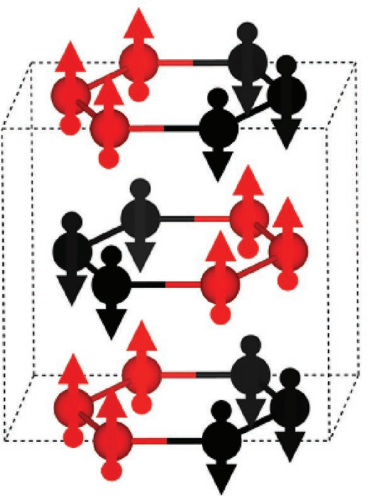

Type III

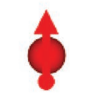

Spin up b)
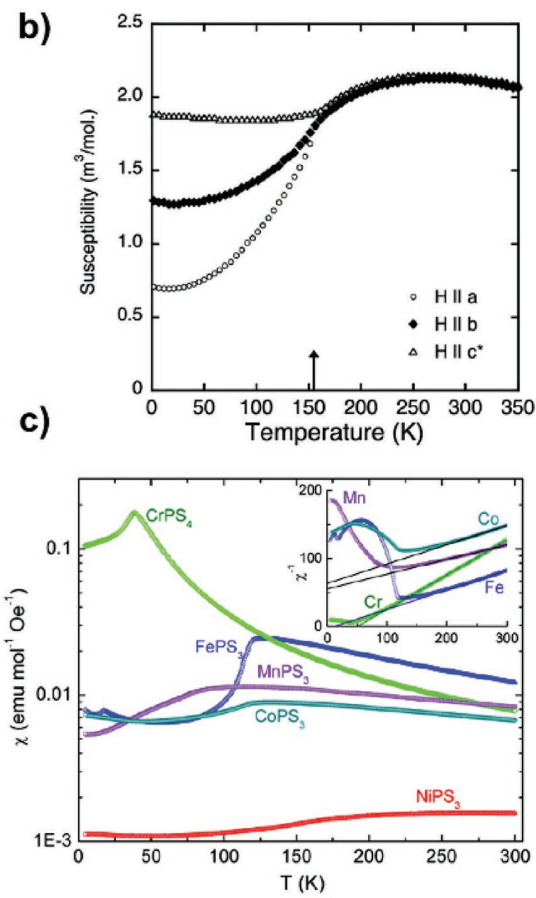

Figure 8. a) The schematic of three published magnetic structures of $\mathrm{MPX}_{3}$. Type I, type II, and type III for NiPS, $\mathrm{MnPS}_{3}$, and FePS , respectively. The moments are on the metal sites. b) Examples of the zero-field-cooled magnetic susceptibility of a $\mathrm{NiPS}_{3}$ crystal measured along some high symmetry directions in an applied magnetic field of $0.1 \mathrm{~T}$. The arrow marks the expected Neel temperature of $155 \mathrm{~K}$. Reproduced with permission. ${ }^{\text {[2] }}$ Copyright 2015, American Physical Society. c) Magnetic susceptibility of the MnPS $, \mathrm{CoPS}_{3}, \mathrm{NiPS}_{3}$, and FePS 3 phases measured at a field of 1000 Oe. The inset is the inverse susceptibility representation (without $\mathrm{NiPS}_{3}$ ). Reproduced with permission. ${ }^{[66]}$ Copyright 2017, American Chemical Society.

in the layer (type II) and their net magnetic moments pointing vertically to the basal planes. In $\mathrm{FePS}_{3}$, each $\mathrm{Fe}^{2+}$ ion is ferromagnetically coupled with two of the three nearest neighbors in single layer. Meanwhile, each plane is anti-ferromagetically coupled to adjacent interplanar ones, which is contrary to the type I structure (type III). Meanwhile, $\mathrm{FePS}_{3}$ has been of interest for its realization of the anti-ferromagnetic $2 \mathrm{D}$ Ising model on a honeycomb lattice. ${ }^{[64,69]}$ As temperature decreases across $\approx 118 \mathrm{~K}$, $\mathrm{FePS}_{3}$ undergoes a conversion from the paramagnetic to the antimagnetic phase. ${ }^{[59]}$ Magnetic measurement shows that bulk $\mathrm{Ni}, \mathrm{Fe}, \mathrm{Mn}$, and Co based $\mathrm{MPS}_{3}$ or $\mathrm{MPSe}_{3}$ have been found to be anti-ferromagnetic with Neel temperatures $\left(T_{N}\right)$ at a range of 82-155 K. ${ }^{[29,39,70]}$ Additionally, the magnetic properties of the $\mathrm{MPX}_{3}$ crystal are affected by the test conditions and magnetic field intensity. Taking the $\mathrm{NiPS}_{3}$ crystal as an example, some previous results demonstrate ${ }^{[64,71]}$ that the susceptibility was a function of temperature and it was anisotropic above $T_{\mathrm{N}}$. Conversely, Wildes et al. ${ }^{[72]}$ found that the susceptibility is isotropic above the $T_{\mathrm{N}}\left(T_{\mathrm{N}}=155 \mathrm{~K}\right)$ while it becomes anisotropic below the $T_{\mathrm{N}}$ with an applied field of $0.1 \mathrm{~T}$ along three high symmetry directions, $a, b$, and $c$ (Figure $8 \mathrm{~b}$ ).

Most bulk $\mathrm{MPS}_{3}(\mathrm{M}=\mathrm{Ni}, \mathrm{Fe}, \mathrm{Co}$, and $\mathrm{Mn})$ compounds are anti-ferromagnetic below their $T_{\mathrm{N}}$ and are paramagnetic above the $T_{\mathrm{N}}$. As is shown in Figure $8 \mathrm{c},{ }^{[29,66]}$ their magnetic susceptibility was conducted at a field of 1000 Oe. Parameters such as apparent total spin S assuming zero orbital moment, the magnetic moment, Neel temperature, and Weiss constant could be evaluated from the susceptibility data. The Weiss constant of $\mathrm{MnPS}_{3}(-245 \mathrm{~K}), \mathrm{CoPS}_{3}(-223 \mathrm{~K})$, and $\mathrm{NiPS}_{3}(-712 \mathrm{~K})$ is negative while it is a positive value for $\mathrm{FePS}_{3}(16 \mathrm{~K})$, indicating the existence of ferromagnetic correlations. However, some compounds, such as $\mathrm{CdPS}_{3}, \mathrm{ZnPS}_{3}$, and $\mathrm{SnPS}_{3}$, in this family exhibit diamagnetism under any temperature because of the core electrons. The magnetic parameters of these bulk MPX compounds are illustrated in Table 4.

The magnetism of the $\mathrm{MPX}_{3}$ crystals in the 2D limit has attracted tremendous attention recently. In this regard, $\mathrm{MPX}_{3}$ monolayers are studied by theoretical calculations except for $\mathrm{FePS}_{3}$ which has an experimental evidence as well. These

Table 4. A list of bulk $\mathrm{MPX}_{3}$ compounds with the known magnetic data.

\begin{tabular}{|c|c|c|c|}
\hline Compound & Type of magnetisma) & Neel temperature ${ }^{\mathrm{b})}[\mathrm{K}]$ & Magnetic moment $[\mu \mathrm{B}]$ \\
\hline $\mathrm{NiPS}_{3}$ & $\mathrm{AFM}^{[27]}$ & $155^{[27]}$ & $3.9^{[49]}$ \\
\hline $\mathrm{MnPS}_{3}$ & $\operatorname{AFM}^{[27]}$ & $78^{[27]}$ & $3.7^{[27]}$ \\
\hline $\mathrm{MnPSe}_{3}$ & $\mathrm{AFM}^{[39]}$ & $74 \pm 2^{[39]}$ & $4.74^{[27]}$ \\
\hline $\mathrm{FePS}_{3}$ & $\mathrm{AFM}^{[39]}$ & $118^{[64]}$ & $5.0^{[27]}$ \\
\hline $\mathrm{FePSe}_{3}$ & $\mathrm{AFM}^{[39]}$ & $119 \pm 7^{[39]}$ & $4.9^{[27]}$ \\
\hline $\mathrm{CoPS}_{3}$ & $\mathrm{AFM}^{[49]}$ & $132^{[49]}$ & - \\
\hline $\mathrm{AgVP}_{2} \mathrm{~S}_{6}$ & $\operatorname{AFM}^{[127]}$ & $10-15^{[127]}$ & - \\
\hline $\mathrm{AgCrP}_{2} \mathrm{~S}_{6}$ & $\operatorname{AFM}^{[128]}$ & $20^{[128]}$ & - \\
\hline $\mathrm{AgCrP}_{2} \mathrm{Se}_{6}$ & $\operatorname{AFM}^{[79]}$ & $42^{[79]}$ & - \\
\hline $\mathrm{CuCrP}_{2} \mathrm{~S}_{6}$ & $\operatorname{AFM}^{[79]}$ & $40^{[79]}$ & - \\
\hline $\mathrm{CuCrP}{ }_{2} \mathrm{Se}_{6}$ & $\operatorname{AFM}^{[1]]}$ & $30^{[1]]}$ & - \\
\hline
\end{tabular}

a,b)The experimental data based on bulk MPX ${ }_{3}$ crystals. 

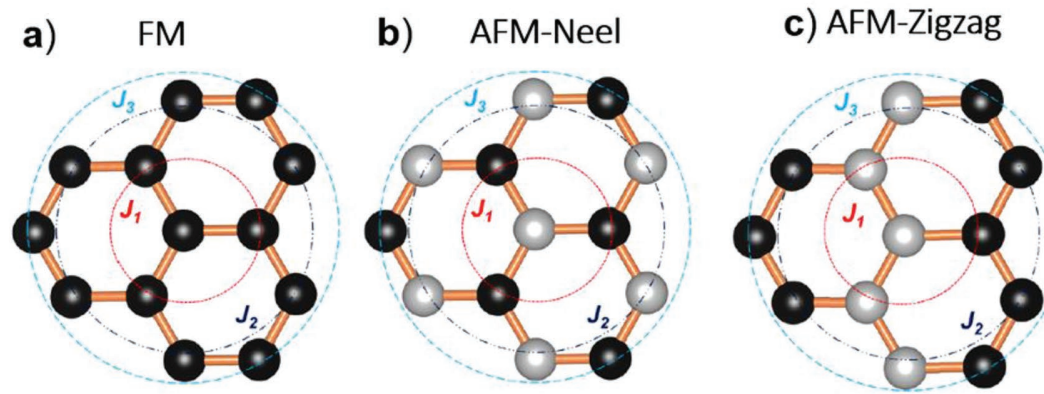

e)

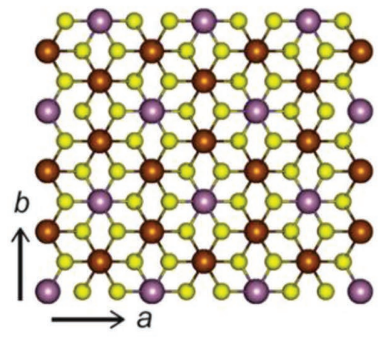

f)

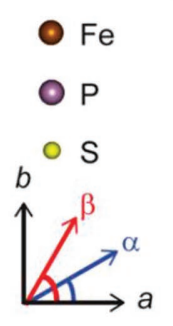

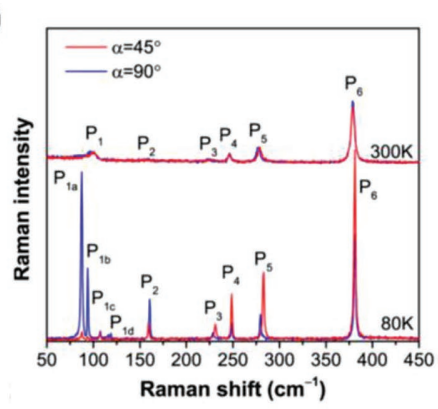

d) AFM-Stripy

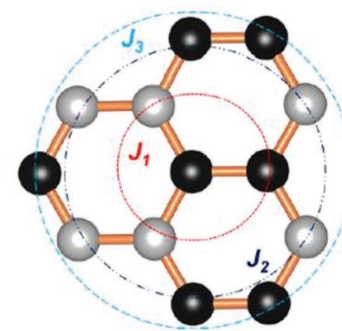

g)

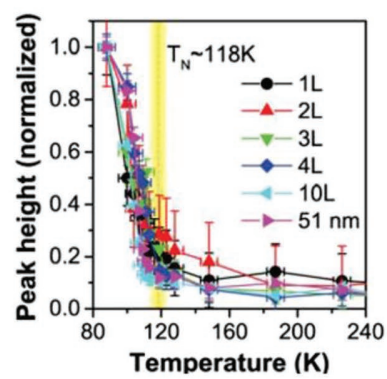

Figure 9. Top view of different magnetic ordering arrangements: a) ferromagnetic, b) Neel anti-ferromagnetic, c) zigzag anti-ferromagnetic, and d) stripy anti-ferromagnetic. The black and white spheres represent up and down spins, respectively. The red circles identify the nearest neighbors (NN) of the central metal site, the navy blue circles (dashed-dotted) identify the second-nearest neighbors, and the light blue circles identify the thirdnearest neighbors. (a)-(d) Reproduced with permission. ${ }^{[54]}$ Copyright 2016, American Physical Society. e) $a-b$ plane of monolayer FePS . Definitions $^{2}$ of the incident $(\alpha)$ and scattered $(\beta)$ polarization angles are shown. $\mathrm{f}$ ) Polarized Raman spectra of bulk FePS 3 at 80 and $300 \mathrm{~K}$ in parallel polarization configurations $(\alpha=\beta)$, for $\alpha=45^{\circ}$ and $90^{\circ}$, respectively. g) Temperature dependence of the $\mathrm{P}_{1 \mathrm{a}}$ peak height at different thicknesses. (e)-(g) Reproduced with permission. ${ }^{[35]}$ Copyright 2016, American Chemical Society.

studies mainly focus on monolayer magnetic ordering, the relationship between band structure and magnetic phase, and modulation techniques. Chittari et al. ${ }^{[5]}$ studied the electronic and magnetic properties of single-layer $\mathrm{MPX}_{3}(\mathrm{M}=\mathrm{V}, \mathrm{Cr}, \mathrm{Mn}$, $\mathrm{Fe}, \mathrm{Co}, \mathrm{Ni}, \mathrm{Cu}$, and $\mathrm{Zn} ; \mathrm{X}=\mathrm{S}$, Se, Te) via first-principles calculations. For the $\mathrm{MPX}_{3}$ monolayer, there are four magnetic orderings (illustrated in Figure 9a-d) which include ferromagnetism (FM), Neel anti-ferromagnetism (AFM), zigzag AFM (zAFM), and stripy AFM. As to the FM ordering, all the spin-states of the metal atoms have the same orientation. In addition, the spinstates in the nearest neighbor metal atoms have an opposite orientation for the Neel anti-ferromagnetic ordering. The zigzag AFM ordering, where the spin-state of the adjacent metal atom chains along the zigzag direction has an opposite orientation, is different from the previous two. However, the spin-state of the adjacent metal atoms for the stripy anti-ferromagnetic ordering chain along the armchair direction has an opposite orientation. According to the DFT results, ${ }^{[54]}$ monolayers $\mathrm{VPX}_{3}, \mathrm{MnPX}_{3}$, and $\mathrm{NiPX}_{3}(\mathrm{X}=\mathrm{S}, \mathrm{Se}$, and Te) prefer the $\mathrm{AFM}$ ordering, while the nonmagnetic state of monolayer $\mathrm{CoPX}_{3}, \mathrm{CuPX}_{3}, \mathrm{ZnPX}_{3}$, and $\mathrm{FePX}_{3}$ is more stable than the others, except for FePS which prefers the zAFM ordering. Beyond that, monolayers $\mathrm{CrPS}_{3}$ and $\mathrm{CrPSe}_{3}$ are ferromagnetic. Recently, several studies on spin properties using Raman spectroscopy have reported on spin-phonon coupling and quasielastic scattering owing to the difficulty in directly measuring the magnetic behaviors of atomically thin $2 \mathrm{D}$ crystals..$^{[59,73]}$ In the Raman spectra of magnetic crystals, the appearance of two magnon scattering and change of the Raman peak positions or intensities suggest ordered spin states. ${ }^{[35,74]}$ Particularly, the changes in the Raman spectrum of anti-ferromagnetic materials concomitant with a magnetic transition are good signals for monitoring their magnetic ordering. Based on this method, Wang et al. ${ }^{[59]}$ verify the magnetic persistence in monolayer $\mathrm{FePS}_{3}$ nanosheets, revealing that the intralayer spin arrangement dominates the magnetic structure. The $\mathrm{FePS}_{3}$ nanosheets with different layers were studied by monitoring the intensity of the Raman peaks $\left(\mathrm{P}_{1 \mathrm{a}}\right)$, which result from zone folding because of AFM ordering at the transition temperature. ${ }^{[35]}$ Lee et al. found that it exhibits an Ising type AFM ordering when its thickness reduces to the monolayer $(0.7 \mathrm{~nm})$ (Figure 9e-g). Moreover, the transition temperature $\left(T_{N} \approx 118 \mathrm{~K}\right)$ remains irrelevant to the thickness of FePS 3 . This result indicates that the weak interlayer interaction plays a negligible role on the anti-ferromagnetic ordering.

Notably, there is a certain correlation between band and magnetic structure of monolayer $\mathrm{MPX}_{3},{ }^{[54]}$ proved by the first principle calculation. Monolayer $\mathrm{MPX}_{3}$ nanosheets are generally metallic with ferromagnetic ordering. Particularly, monolayer $\mathrm{CoPX}_{3}$ turned out to be nonmagnetic. The band structures of various monolayer $\mathrm{MPX}_{3}$ (Figure 10a) show that the nonmagnetic ones (with green bands) can be metal (Co-based and Cu-based $\mathrm{MPX}_{3}$ compounds), semiconductor $\left(\mathrm{FePX}_{3}\right)$, or insulator $\left(\mathrm{ZnPX}_{3}\right)$. The semiconducting ones, such as $\mathrm{VPX}_{3}$, $\mathrm{MnPX}_{3}, \mathrm{NiPX}_{3}$, and $\mathrm{FePX}_{3}$, exhibit anti-ferromagnetic ordering, while, Cr-based compounds show ferromagnetic ordering with a metallic phase. 
a)

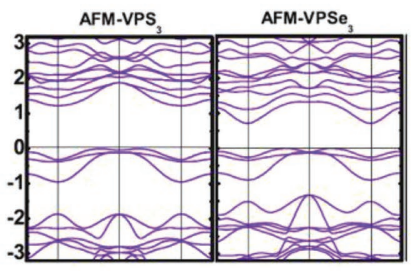

AFM-MnPS

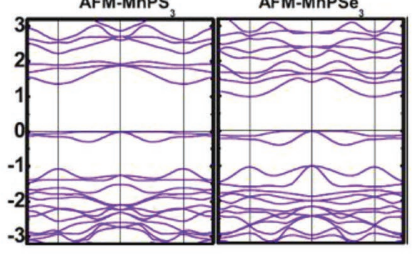

b)

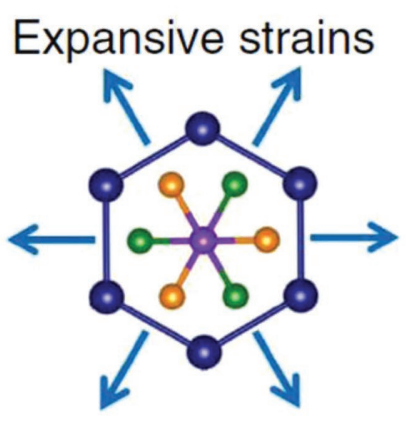

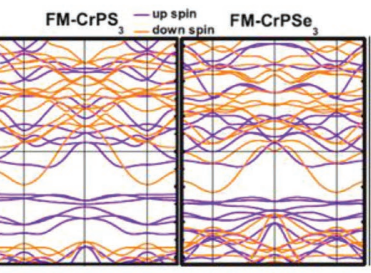

ZAFM-FePS,
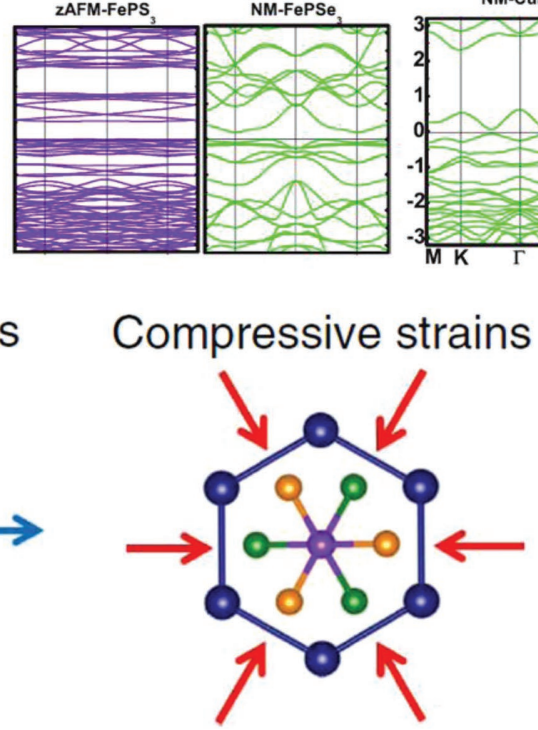
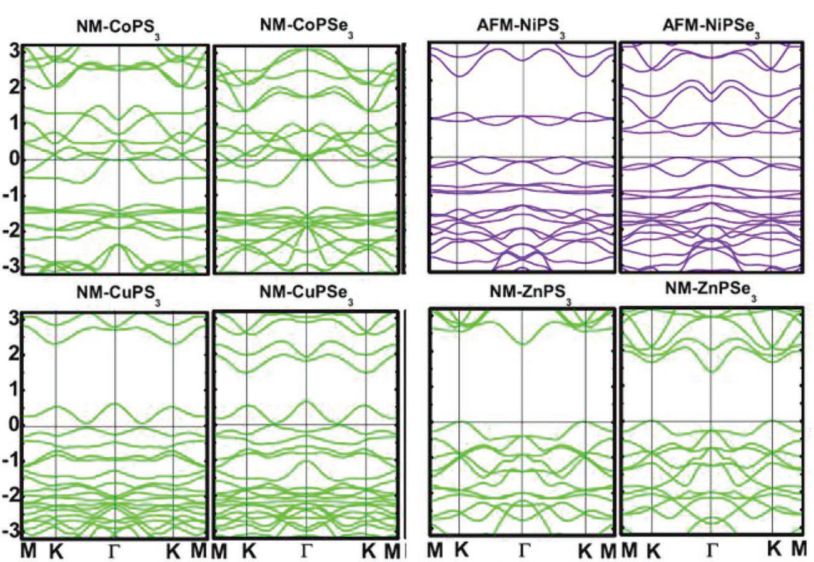

NM-ZnPS

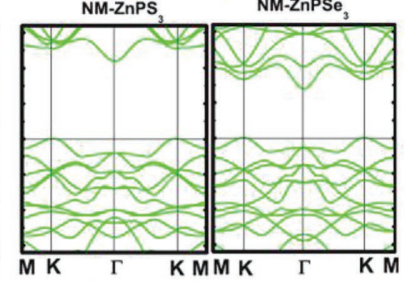

c)

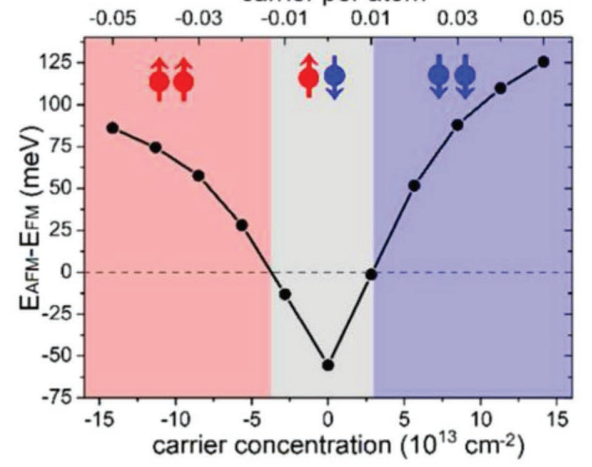

Figure 10. a) Band structures for single-layer $\mathrm{MPX}_{3}(\mathrm{M}=\mathrm{V}, \mathrm{Cr}, \mathrm{Mn}, \mathrm{Fe}, \mathrm{Co}, \mathrm{Ni}, \mathrm{Cu}$, and $\mathrm{Zn} . \mathrm{X}=\mathrm{S}, \mathrm{Se})$ compounds in their lowest-energy magnetic configuration. The bands are violet for AFM configurations, violet and orange for the up and down split spin bands in the FM configurations, and green for the NM phases. b) Influence of in-plane strain on the magnetic configurations of selected $\mathrm{MPX}_{3}$ compounds. Reproduced with permission. ${ }^{[5]}$ Copyright 2016, American Physical Society. c) Relative energy of AFM and FM states under the variation of carrier concentration for 2D MnPSe ${ }_{3}$. The positive and negative values are for electron and hole doping, respectively. The up and down arrows indicate up-spin and down-spin, respectively. Reproduced with permission. ${ }^{[28]}$ Copyright 2014, American Chemical Society.

The magnetic phase transition can also be realized through applying stress or modulating the carrier concentration. Chittari et al. ${ }^{[54]}$ found that applied strains can act as switchers to give rise to transitions or change in the stability of the magnetic phases. The compressive and expansive biaxial strains can be obtained through enlarging rectangular unit cell. Similarly, scaling either in the zigzag or armchair directions is used to obtain the uniaxial strains (Figure 10b). Theoretically, huge strains are required to tune the magnetic behavior of $\mathrm{MnPS}_{3}$. However, for other systems, such as $\mathrm{CrPS}_{3}, \mathrm{FePS}_{3}, \mathrm{FePSe}_{3}$, and $\mathrm{NiPS}_{3}$, the transitions of magnetic properties can be realized when a small strain is applied. Moreover, Yang and coworkers ${ }^{[28]}$ predicted that controlling the carrier concentration will lead to a magnetic phase transition from the AFM phase at the ground state to the FM phase after the observation is made via increasing the density of the carriers $\left(1.4 \times 10^{14} \mathrm{~cm}^{-2}\right)$ on the $\mathrm{MnPSe}_{3}$ nanosheet (Figure 10c).

Alloying is also an approach to alter the magnetic properties for these $\mathrm{MPX}_{3}$ materials. The nonmagnetic metal element (Mn) is alloyed with $\mathrm{ZnPS}_{3}$ to obtain the $\mathrm{Mn}_{x} \mathrm{Zn}_{1-x} \mathrm{PS}_{3}$ crystal. ${ }^{[75]}$ The alloying extent, namely, the value of $x$, usually has an influence on the value of $T_{\mathrm{N}}$ and the magnetic phase of the alloyed $\mathrm{MPX}_{3}$. The study of the $\mathrm{Mn}_{x} \mathrm{Zn}_{1-x} \mathrm{P}_{1} \mathrm{~S}_{3}{ }^{[75]}$ crystal demonstrates that its $T_{\mathrm{N}}$ value is related to the concentration of $\mathrm{Zn}$ atoms. It decreases concomitantly when the concentration of the nonmagnetic cation species increases. A $T_{\mathrm{N}}$ /appliedfield(Hz)/dilution magnetic phase diagram for $\mathrm{Mn}_{x} \mathrm{Zn}_{1-x} \mathrm{P}_{2} \mathrm{~S}_{6}$ is shown in Figure 11a. In this diagram, the enclosed region is the AFM region with a percolation threshold of $x=0.7$. A high-order paramagnetic phase and a disordered paramagnetic phase will appear with a large magnetic field and high temperature, respectively. Likewise, the low percentage $(x<0.7)$ of $\mathrm{Mn}$ atoms also introduces the paramagnetic phase. Above the top surface of the AFM region, the spin-flop phase dominates the diagram. The other is alloying $\mathrm{MPX}_{3}$ with magnetic metal for $\mathrm{Mn}_{1-x} \mathrm{Fe}_{x} \mathrm{PS}_{3}{ }^{[76]}$ and $\mathrm{Fe}_{0.5} \mathrm{Ni}_{0.5} \mathrm{PS}_{3}{ }^{[68]}$ The substitutive magnetic metal atom, like $\mathrm{Mn}$ and Fe atoms, in alloyed $\mathrm{MPX}_{3}$ is more likely to bring about a magnetic glass phase or a spin glass phase. The alloyed $\mathrm{Mn}_{1-x} \mathrm{Fe}_{x} \mathrm{PS}_{3}$ exhibits a spin glass state when a portion of the $\mathrm{Mn}$ atoms are substituted by other magnetic Fe atoms (Figure 11b). ${ }^{[76]}$ It is clear that the magnetic phase of $\mathrm{Mn}_{1-x} \mathrm{Fe}_{x} \mathrm{PS}_{3}$ changes with the increase of the iron concentration. Obviously, the anti-ferromagnetic phases exist in the ranges of $0.0 \leq x \leq 0.2$ and $0.8 \leq x \leq 1.0$. The spin glass state is in the range of $0.5 \leq x \leq 0.6$ and the reentrant spin glass state is confined to the ranges of $0.3 \leq x \leq 0.45$ or $0.6 \leq x \leq 0.7$ at a low temperature. Apart from the spin glass state, a magnetic glass state may appear in a magnetically substituted $\mathrm{MPX}_{3}$ system. Goossens et al. ${ }^{[68]}$ found that this alloyed system shows obvious hysteresis for $\mathrm{Fe}_{0.5} \mathrm{Ni}_{0.5} \mathrm{PS}_{3}$ in the transition temperature and 
a)

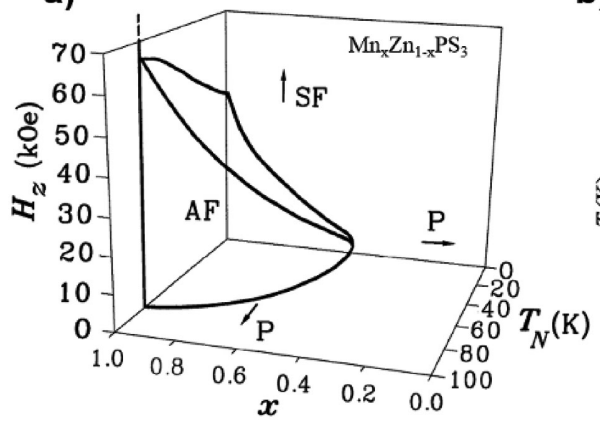

b)

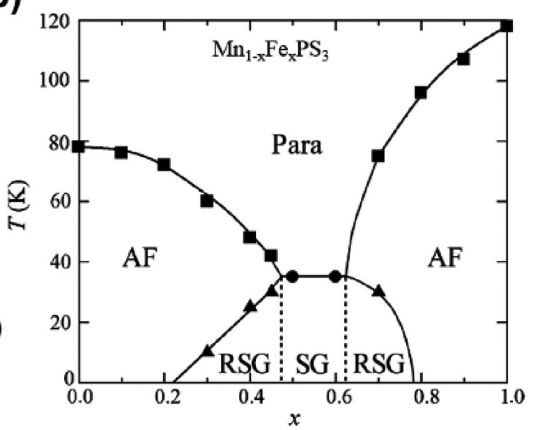

c)

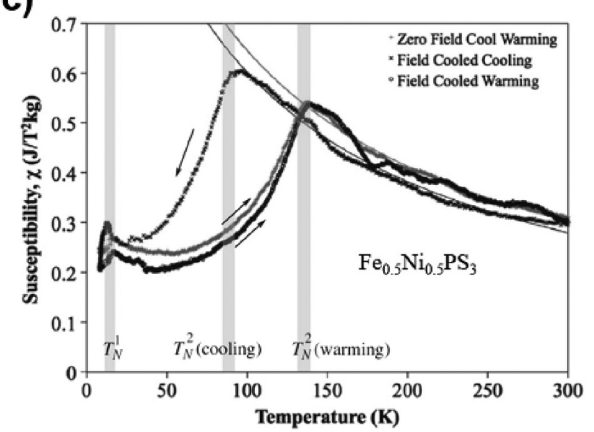

Figure 11. a) The temperature/applied-field $\left(\mathrm{H}=\mathrm{H}_{\mathrm{Z}}\right)$ /dilution phase diagram for $\mathrm{Mn}_{x} \mathrm{Zn}_{1-x} \mathrm{PS}$. SF, AF, and $\mathrm{P}$ stand for the spin-flop, anti-ferromagnetic, and paramagnetic phases, respectively. Reproduced with permission. ${ }^{[75]}$ Copyright 1998, IOP Publishing. b) Magnetic phase diagram of the $\mathrm{Mn}_{1-x} \mathrm{Fe}_{x} \mathrm{PS}_{3}$. The notations SG, RSG, AF, and Para show a spin glass, a re-entrant spin glass, an anti-ferromagnetic ordered, and a paramagnetic phase, respectively. Reproduced with permission. ${ }^{[76]}$ Copyright 2007, Elsevier B.V. c) Magnetic susceptibility of a Fe ${ }_{0.5} \mathrm{Ni}_{0.5} \mathrm{PS}_{3}$ powder, measured in a field of 1 T under a range of conditions. The directions of temperature change were as follows: (1) warming after cooling in the zero field (ZFCW); (2) cooling in a field (FCC); and finally (3) warming after cooling in a field (FCW). Reproduced with permission. ${ }^{[68]}$ Copyright 2013, Elsevier B.V.

also related to the direction of temperature change (Figure 11c). Two Neel temperatures of 14 and $97 \mathrm{~K}$ or $\approx 138 \mathrm{~K}$, referring to $T^{1}{ }_{\mathrm{N}}$ and $T^{2}{ }_{\mathrm{N}}$, were found, and three magnetic phases could be distinguished from the measured susceptibility of $\mathrm{Fe}_{0.5} \mathrm{Ni}_{0.5} \mathrm{PS}_{3}$. Meanwhile, there is a distinct thermal hysteresis in the field cooled cooling (FCC) and field cooled warming (FCW) susceptibilities. The FCC state also transforms into zero field cooled warming $(\mathrm{ZFCW}) / \mathrm{FCW}$ states after relaxation over a period of $\approx 2 \mathrm{~h}$. These results reveal the existence of magnetic glass. ${ }^{[68]}$

\subsubsection{Ferroelectricity}

One of the interesting phenomena in metal thiophosphates or selenophosphates is the presence of ferroelectric ordering. ${ }^{[4,77,78]}$ Usually, the ferroelectric ordering only appears on $\mathrm{M}_{1} \mathrm{M}_{\mathrm{II}} \mathrm{P}_{2} \mathrm{X}_{6}$ crystals, such as $\mathrm{CuInP}_{2} \mathrm{~S}_{6},{ }^{[44]} \mathrm{CuInP}_{2} \mathrm{Se}_{6},{ }^{[79]} \mathrm{CuBiP}_{2} \mathrm{Se}_{6},{ }^{[48]}$ and $\operatorname{AgBiP}_{2} \mathrm{~S}_{6} \cdot{ }^{[48]} \mathrm{A}$ list of all $\mathrm{M}_{\mathrm{I}} \mathrm{M}_{\mathrm{II}} \mathrm{P}_{2} \mathrm{X}_{6}$ compounds and their ferroic ordering is illustrated in Table 5. Especially, the ferroelectric ordering of $\mathrm{CuInP}_{2} \mathrm{~S}_{6}$ has been widely studied. The structure of $\mathrm{CuInP}_{2} \mathrm{~S}_{6}$ is shown in Figure $12 \mathrm{a}$. $\mathrm{Cu}^{\mathrm{I}}$ atoms can move among three sites, as follows: the first site is closest to the center of the octahedron (Cu1), the second site is further off-center (Cu2), and the third site is in the interlayer space (Cu3). ${ }^{[80]}$ The transition from order to disorder occurs at $315 \mathrm{~K}^{[44]}$ with indirect evidence for copper hopping motions. Above the temperature of $315 \mathrm{~K}$, the symmetry of the $\mathrm{CuInP}_{2} \mathrm{~S}_{6}$ phase is apparent because the first site has two positions, i.e., "up" and "down" positions

Table 5. A list of $M^{\prime} M^{\prime \prime \prime} P_{2} X_{6}$ compounds with known ferroic ordering.

\begin{tabular}{lccc}
\hline Compound & Ferroically ordered type & Ordering temperature $\left[T_{c}, \mathrm{~K}\right]$ & Ref. \\
\hline $\mathrm{CuCrP}_{2} \mathrm{~S}_{6}$ & Anti-ferrielectric & 150 & {$[17]$} \\
CulnP$_{2} \mathrm{~S}_{6}$ & Ferrielectric & 315 & {$[19]$} \\
$\mathrm{CuInP}_{2} \mathrm{Se}_{6}$ & Ferrielectric & 236 & {$[79]$} \\
$\mathrm{CuVP}_{2} \mathrm{~S}_{6}$ & - & 20 & {$[81]$} \\
$\mathrm{CuBiP}_{2} \mathrm{Se}_{6}$ & Anti-ferrielectric & $93<T_{\mathrm{c}}<173$ & {$[48]$} \\
$\mathrm{AgBiP}_{2} \mathrm{Se}_{6}$ & Anti-ferrielectric & $T_{\mathrm{c}}<295$ & {$[48]$} \\
\hline
\end{tabular}

(Figure 12b), and the third site shows an equal position of both "up" and "down". ${ }^{[4]}$ When the $\mathrm{CuInP}_{2} \mathrm{~S}_{6}$ is cooled below $315 \mathrm{~K}$ (Figure 12c), the motion of the intersite $\mathrm{Cu}$ is restricted to lock the $\mathrm{Cu}^{1+}$ sublattice in the "up" position with a compensatory shift of the $\mathrm{In}^{3+}$ sublattice to the opposite polarity, leading to the formation of a non-centrosymmetric ferroelectric phase. An obvious symmetry transition is from the paraelectric phase to the ferroelectric phase when the temperature is $315 \mathrm{~K}$. Additionally, small amounts of element substitution either of the In or $\mathrm{S}$ site will lead to intriguing regulation in the nature properties. If the Se content is increased in $\mathrm{CuInP}_{2}\left(\mathrm{~S}_{x} \mathrm{Se}_{1-x}\right)_{6}$, the regulation from ferroelectric to a dipolar glass or other phase is easily triggered. ${ }^{[77]}$ Similarly, the $\mathrm{CuVP}_{2} \mathrm{~S}_{6}$ crystal is also found to exhibit ordered displacement of metal ions at a low temperature. The $\mathrm{Cu}^{\mathrm{I}}$ in the trigonal site forming acentric C2 symmetry will be frozen below $20 \mathrm{~K} .{ }^{[81]}$

In addition, hydrostatic pressure is also applied to realize the structural phase transition of $\mathrm{CuInP}_{2} \mathrm{~S}_{6}$. Its ferroelectric transition temperature $\left(T_{c}\right)$, demonstrating the linear increment along with the strength of pressure $\left(\partial T_{\mathrm{c}} / \partial P=210 \mathrm{~K} \mathrm{GPa}^{-1}\right)$, can be tuned with pressure. ${ }^{[82]}$ This result indicates the transition from order to disorder nature and the coupling effect of ferroelectric dipoles across the vdW gap. Notably, the ferroelectric polarization can occur in $\mathrm{CuInP}_{2} \mathrm{~S}_{6}$ crystal when the temperature is above the room temperature. This is unique for the vdW layered crystals. The domain structure, switchable polarization, and hysteresis loops in the ambient and ultrahigh vacuum scanning probe microscopy spectra, studied by Belianinov et al., ${ }^{[83]}$ directly reveal stable, ferroelectric polarization of this crystal. They also found that the domain structure of flakes, whose thickness is $>100 \mathrm{~nm}$, is similar to the cleaved bulk surfaces, whereas polarization disappears when its thickness is below $50 \mathrm{~nm}$, which is originated from the instability of polarization under the depolarization field. The piezoresponse force microscopy studies show that the polarization switching at high bias is also related to the ionic mobility and the ferroelectric domains are persistent across several hundreds of layers. ${ }^{[83,84]}$ The ferroelectric properties are also shown in the $\mathrm{MPSe}_{3}$ class. The $\mathrm{P}_{2} \mathrm{Se}_{6}$ bond possesses a weaker degree of covalence than that in $\mathrm{P}_{2} \mathrm{~S}_{6}$ bonds. Thus, the $\mathrm{Cu}^{\mathrm{I}}$ ion displacement occurs at the lower 
a)

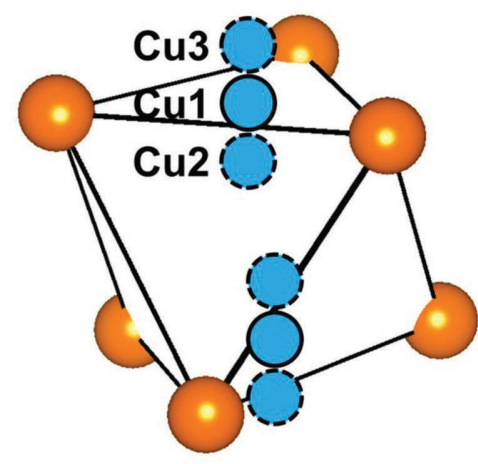

b)

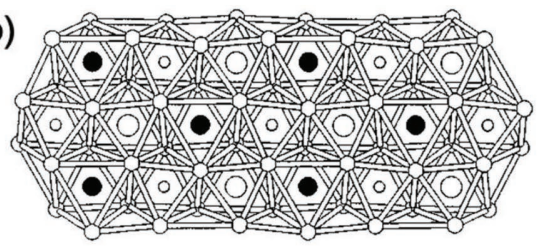

c)

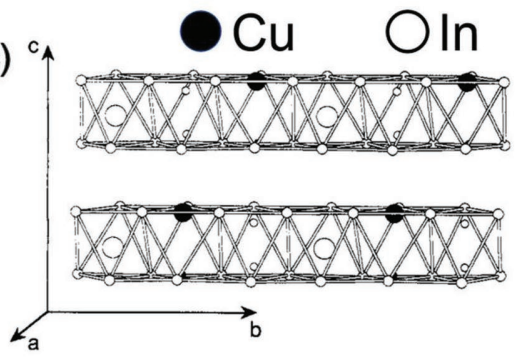

Figure 12. a) The position of three "up" copper sites labeled $\mathrm{Cu}^{1}$ (the off-center), $\mathrm{Cu}^{2}$ (the almost central), $\mathrm{Cu}^{3}$ (the interlayer site). The corresponding "down" sites are also shown. b) Projection onto the $a-b$ plane of the $\mathrm{CuInP}_{2} \mathrm{~S}_{6}$ structure showing the triangular sublattices formed by the copper and indium cations and the P-P pairs. c) Two layers of Culn $\mathrm{P}_{2} \mathrm{~S}_{6}$ shown in the ferroelectric phase $(T<315 \mathrm{~K})$. The up (down) shifted $\mathrm{Cu}^{\prime}\left(\mathrm{In}^{\prime \prime \prime}\right)$ ions are represented by the larger black (white) ball, and the smaller white circles are the P. (a)-(c) Reproduced with permission. ${ }^{[44]}$ Copyright 1997, American Physical Society.

temperature phase. ${ }^{[44,79]}$ Phase transitions in the $\mathrm{CuInP}_{2} \mathrm{Se}_{6}$ compound contain a second-order phase transition at $248 \mathrm{~K}\left(\mathrm{~T}_{\mathrm{i}}\right)$ and a first order transition at $236 \mathrm{~K}\left(T_{c}\right)$, which is lower than that $\left(T_{c}=315 \mathrm{~K}\right)$ in $\mathrm{CuInP}_{2} \mathrm{~S}_{6} \cdot{ }^{[44]}$

\subsubsection{Intercalation Effect}

Intercalation is a reversible chemical process to insert foreign species, including molecules and ions, into the crystal gap. The remarkably wide van der Waals gaps existing in $\mathrm{MPX}_{3}$ compounds create a suitable platform for practical realization of intercalation chemistry. For instance, alkali metals are shown to chemically and electrochemically intercalate into the $\mathrm{MPX}_{3}$ crystals. ${ }^{[85]}$ Among them, $\mathrm{NiPS}_{3}, \mathrm{FePS}_{3}$, and $\mathrm{CoPS}_{3}$ can easily react with butyl-lithium, whereas $\mathrm{CdPS}_{3}, \mathrm{ZnPS}_{3}$, and $\mathrm{MnPS}_{3}$ do not. Considering the structure of $\mathrm{MPS}_{3}$ crystals, filling of all the octahedral sites in the vdW gaps by the lithium cations should lead to the formation of product $\mathrm{Li}_{1.5} \mathrm{MPS}_{3}$ (i.e., $\mathrm{Li}_{3} \mathrm{M}_{2} \mathrm{P}_{2} \mathrm{~S}_{6}$ ). At the same time, their magnetic, optical, and electrical properties after lithium intercalation were investigated in detail. ${ }^{[49]}$ The intercalation can result in the occurrence of negative free carriers and a decrement of magnetic susceptibility in $\mathrm{Li}_{x} \mathrm{NiPS}_{3}$. In addition to these fundamental interests, they can be used in lithium batteries. ${ }^{[23,86]} \mathrm{A}$ high formation energy, which is related to the difference between the $\mathrm{Li}^{+} / \mathrm{Li}$ potential and the accepting $d$ level, for the intercalation trigger, a high potential of the host compound. ${ }^{[87,88]}$ Thus, the nickel phase was proved to be best candidate used in Li batteries. On the one hand, the ideal size of $\mathrm{vdW}$ gap presents the appropriate space to accommodate the $\mathrm{Li}^{+}$ions without expansion. The unchanged cell volume is a strong advantage of the $\mathrm{MPS}_{3}$ materials used as a cathode. On the other hand, the chemistry of $\mathrm{MPX}_{3}$ compounds has an ionic nature and, hence, they can be viewed as ionic salts with the metal ions being connecting together by $\left[\mathrm{P}_{2} \mathrm{~S}_{6}\right]^{4-}$ bridging ligands. ${ }^{[29]}$ Therefore, the high mobility of the metal cations in the ion-exchange mechanism describes the intercalation.

\section{Synthesis Methods}

The fundamental exploration of functional materials with respect to their properties and applications greatly depends on the development of facile and reliable synthesis methods. Particularly, regarding the case of 2D layered materials, the achievement of an atomic thickness with a lateral size of up to micrometers is sought to realize the interesting properties, especially the unique magnetic and electronic properties. Historically, the attempt to synthesize $\mathrm{MPX}_{3}$ materials can be traced back to the late 19th century, which was reported by Friedel ${ }^{[17]}$ who obtained $\mathrm{FePS}_{3}$ by heating a weighed amount of phosphorus pentasulfide with iron. Later, Ferrand ${ }^{[19]}$ was then able to extend this technique for growing $\mathrm{ZnPS}_{3}, \mathrm{CdPS}_{3}$, and $\mathrm{NiPS}_{3}$ materials. Though investigation of $\mathrm{MPX}_{3}$ was held back till the introduction of the vapor growth method by Klingen et al. in the mid1960s. ${ }^{[89]}$ Yet, the obtained products using the vapor phase or hydrothermal growth methods ${ }^{\left[{ }^{[0]}\right.}$ had micro size. Very recently, techniques such as chemical vapor deposition and exfoliation were introduced in which ultrathin materials of this family were communicated with interesting applications. In the subsequent section, we present the strategies and progress made for growing $\mathrm{MPX}_{3}$ materials.

\subsection{Exfoliation and Intercalation}

Micromechanical cleavage is usually used in fabricating ultrathin nanosheets by exfoliating layered bulk crystals by using Scoch tape or force-assisted liquid exfoliation. ${ }^{[60,91,92]}$ In this regard, the cleavage energies of layered $\mathrm{MPX}_{3}$ and the formation energies of single-layer $\mathrm{MPX}_{3}$ are essential properties that need to be considered. Du et al. and Liu et al. ${ }^{[20,93]}$ have calculated the cleavage and the formation energies of some 2D layered $\mathrm{MPX}_{3}$ materials, respectively. The cleavage energies have been calculated by determining the separation beteween the fractured layer $(d)$ and the original layer $\left(d_{0}\right)$. As shown in Figure 13a, the trend of increasing cleavage energy is associated with the enlargment of the interlayer separation and ultimately reaches the maximum level. Of note, the cleavage energy of these layerd $\mathrm{MPX}_{3}$ materials, except for $\mathrm{FePS}_{3}$, is smaller than that of graphite $\left(\approx 0.36 \mathrm{~J} \mathrm{~m}^{-2}\right),{ }^{[28]}$ indicating the suitability of layerd $\mathrm{MPX}_{3}$ materials for exfoliation (Figure 13b). Additionally, the formation energy of layered $\mathrm{MPX}_{3}$ material is much lower than that of a single-layer $\mathrm{MoS}_{2}\left(\approx 0.14 \mathrm{~J} \mathrm{~m}^{-2}\right)$, that is to say, less formation energy is needed in the process of exfoliation from the bulk materials to single-layer nanosheets (Figure 13c). 

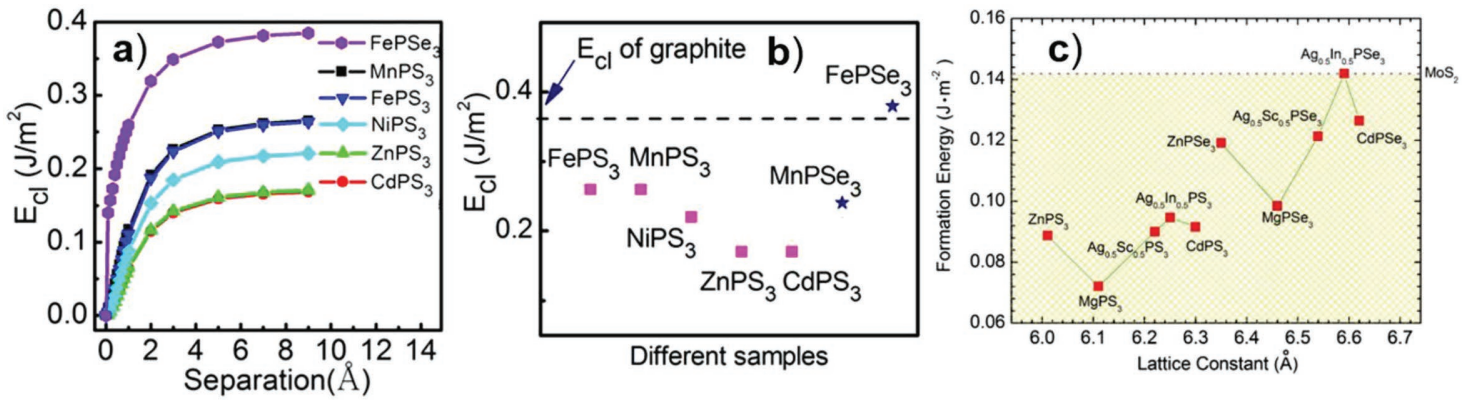

Figure 13. a) Cleavage energy versus the separation distance. b) Comparison of the saturated cleavage energy between graphite and the compounds $\mathrm{MPS}_{3}$ (pink squares) and $\mathrm{MPSe}_{3}$ (blue stars). Reproduced with permission. ${ }^{[20]}$ Copyright 2015, American Chemical Society. c) Calculated formation energies of single-layer $\mathrm{MPX}_{3}$ as a functional of the corresponding lattice constants $(\AA)$, compared with that of $\operatorname{MoS}_{2}$. The blue dashed line indicates the formation energy of $\mathrm{MoS}_{2}$. Reproduced with permission. ${ }^{[93]}$ Copyright 2014, AIP Publishing LLC.

Therefore, both the small values of cleavage and formation energies of layerd $\mathrm{MPX}_{3}$ materials determine the success in exfoliation of bulk $\mathrm{MPX}_{3}$ crystals to single layered ones.

The successful preparation of graphene by the "Scotch Tape"method ${ }^{[91]}$ has prompted researchers to comprehensively study lots of layered materials. Inspired by this, the micromechanical cleavage technique has been widely utilized to obtain various types of ultrathin nanosheets from the corresponding layered bulk crystals, including TMD (e.g., $\mathrm{MoS}_{2}, \mathrm{WS}_{2}, \mathrm{MoSe}_{2}$, $\mathrm{WSe}_{2}$, etc.), ${ }^{[94]} \mathrm{MPX}_{3}$ (e.g., $\mathrm{MnPS}_{3}, \mathrm{FePS}_{3}, \mathrm{NiPS}_{3}$, etc.), ${ }^{[20,21,54,95]}$ topological insulators (e.g., $\mathrm{Bi}_{2} \mathrm{Se}_{3}, \mathrm{Bi}_{2} \mathrm{Te}_{3}, \mathrm{Sb}_{2} \mathrm{Te}_{3}$, etc.), ${ }^{[96]}$ antimonene, ${ }^{[97]}$ and $h$-BN. ${ }^{[98]}$ Among the 2D materials preparation routes, this method can be regarded as a nondestructive technique due to the mere physical phenomena (no chemical reaction) occurring during exfoliation. Therefore, successfully exfoliated 2D materials exhibit a clean surface, maintaining the "perfect" crystal quality with little defect site. Moreover, the exfoliations can be made in such a way that the lateral size of the obtained material can be up to dozens of micrometers. Recently, the micromechanical exfoliations of $\mathrm{MPX}_{3}$ materials have been widely reported. As shown in Figure 14a,b,
Du et al. ${ }^{[20,93]}$ successfully exfoliated bulk $\mathrm{FePS}_{3}$ crystal to fewlayered nanosheets by the "cotch Tape" method. This result can be further confirmed with an optical microscopy photograph and the corresponding AFM topography (Figure 14c,d). Unfortunately, the production yield from micromechanical cleavage technique is quite low with inferior precision and there is restricted controllability for practical application. Additionally, liquid exfoliation has been widely adopted to produce $\mathrm{MPX}_{3}$ nanosheets. Typically, it requires the addition of some small molecules, such as acetone, lithium ions, and surfactants, ${ }^{[99-101]}$ to assist the process of exfoliation. These types of molecules are able to percolate into the interlayer matrix to expand the van der Waals gaps. Figure 14e depicts a schematic illustration of the small molecule assisted liquid exfoliation of $\mathrm{MPX}_{3}$ bulk crystals. The scanning electron microscopy (SEM) image of $\mathrm{FePS}_{3}$ bulk crystals and exfoliated $\mathrm{FePS}_{3}$ nanosheets (Figure 14f,g) corroborate the thinning effect of solvent exfoliation. ${ }^{[102]}$ However, impurities and more defective edge sites would be introduced into the $\mathrm{MPX}_{3}$ nanosheets. This method is not an optimal one for fabricating (photo)electronic devices based on $\mathrm{MPX}_{3}$ semiconductors where impurities and defects are detrimental for realizing of excellent
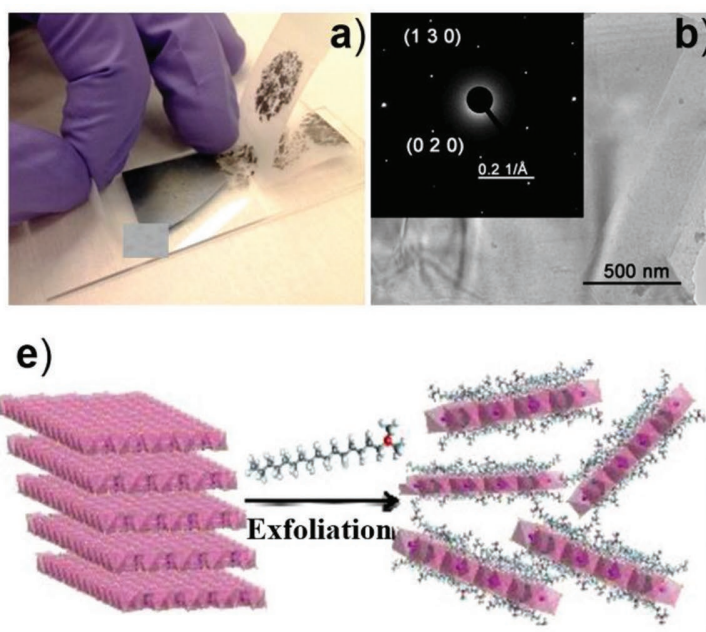
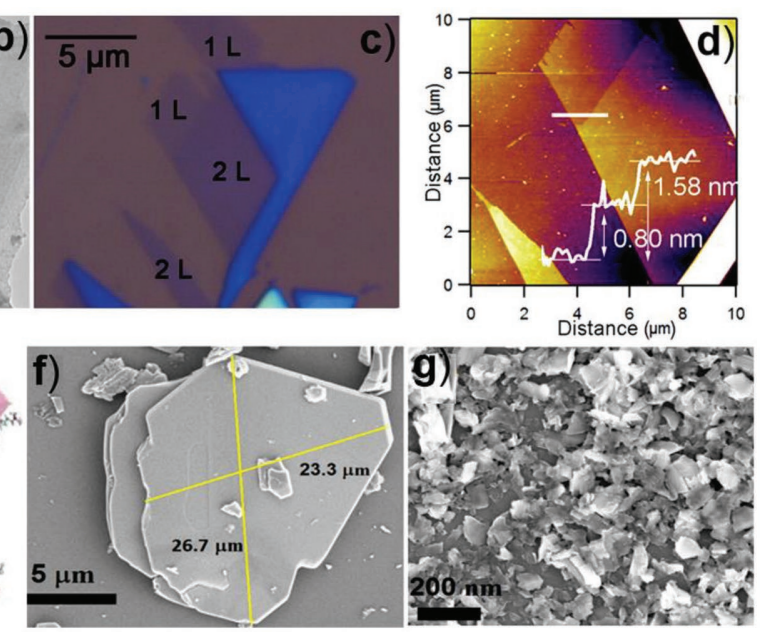

Figure 14. a) Schematic illustration of the typical process with Scotch tape. Reproduced with permission. ${ }^{[129]}$ Copyright 2015, American Chemical

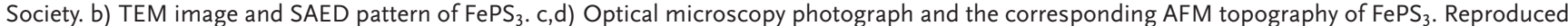

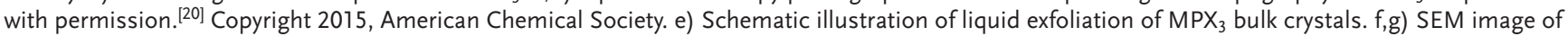
$\mathrm{FePS}_{3}$ bulk crystals and exfoliated FePS 3 nanosheets. Reproduced with permission. ${ }^{[102]}$ Copyright 2016, American Chemical Society. 
performance. In the field of catalysts, the controllable introduction of impurities and defect sites are believed to enhance the catalytic activity. It, therefore, appears reasonable to suggest that if high yield fabrication of the catalyst via solvent exfoliation is assured, it may lead to excellent catalytic activity.

\subsection{Chemical Vapor Growth}

A vast majority of $\mathrm{MPX}_{3}$ compounds with large enough crystals to enable adequate physical characterizations were synthesized through a vapor phase growth technique, i.e., chemical vapor transport (CVT). Stoichiometric amounts of sources, including metal, chalcogen, and phosphorus powders, are located in quartz ampoule along with a transport agent (e.g., iodine) and heated at certain temperature required for the formation of the desired $\mathrm{MPX}_{3}{ }^{[103]}$ The ampoule is made in such a way that it could host a temperature gradient system, such as T1 and T2 $(\mathrm{T} 1<\mathrm{T} 2)$, as shown in Figure 15a. In the hotter region (T2), the precursors form vapors to be transported to the cooler region (T1) for undergoing reaction. The temperature of the cooler region should be thermodynamically efficient for making the growth of $\mathrm{MPX}_{3}$ possible, thereby forming a large plate of crystals (Figure 15b). The heating rate is an important parameter in CVT to widen the interlayer distances and control how well the crystallinity is ordered. Ismail et al. ${ }^{[104]}$ adopted the heating rates of 1 and $40{ }^{\circ} \mathrm{C} \mathrm{min}-1$ to grow $\mathrm{FePS}_{3}$ at $650{ }^{\circ} \mathrm{C}$ through the CVT. It was found that a slow heating rate is useful to get the FePS 3 with single crystalline phase while the fast heating rate was not. Thus, the slow heating rate induces better crystallinity. As seen from the scanning electron microscopic image (Figure 15c-f), this technique generally gives bulk materials with compact layers of irregular thicknesses. A comprehensive investigation of layered metal phosphorus trisulfides/ selenides was recently made by Pumera and co-workers. ${ }^{[66,105]}$ It can be seen that the growth of different members (with varying the metal or the chalcogen) in the $\mathrm{MPX}_{3}$ family requires separate optimization of the temperature and heating rate. Figure $15 \mathrm{~g}$ depicts the Raman spectra as a confirmation tool for the formation of the desired product through the CVT method with appropriate heating rates. The CVT grown $\mathrm{MPS}_{3}$ phases are featured by prominent Raman modes from $\mathrm{P}_{2} \mathrm{~S}_{6}$ units with D3d symmetry (3A1g and 5Eg Raman active modes). In some cases, a pregrown metal oxide/hydroxide precursor is used as a metal source, which is then mixed with a stoichiometric amount of phosphorus and slight in excess of chalcogen in a quartz ampoule. Liang et al. ${ }^{[23]}$ made use of this method to grow $\mathrm{FePS}_{3}, \mathrm{CoPS}_{3}$, and $\mathrm{NiPS}_{3}$. The corresponding precursors of metal hydroxide nanosheets were first synthesized via the hydrothermal method then eventually converted to the $\mathrm{MPX}_{3}$ form at $520{ }^{\circ} \mathrm{C}$ after being mixed with red phosphorus and sulfur powders. Accordingly, the products of $\mathrm{FePS}_{3}, \mathrm{CoPS}_{3}$, and $\mathrm{NiPS}_{3}$ with high crystalline nanosheets, having average thickness and lateral sizes of $\approx 18$ and $\approx 200 \mathrm{~nm}$, respectively, were obtained. The main drawback of CVT is the long duration of the synthesis process. This can be associated with the slow heterogeneous chemical reaction between the metal, chalcogen, and phosphorus atoms. If exactly the stoichiometric amount is taken at the initial stage, the formation kinetics for $\mathrm{MPX}_{3}$ slows down when the pressure of the gaseous product becomes slow.
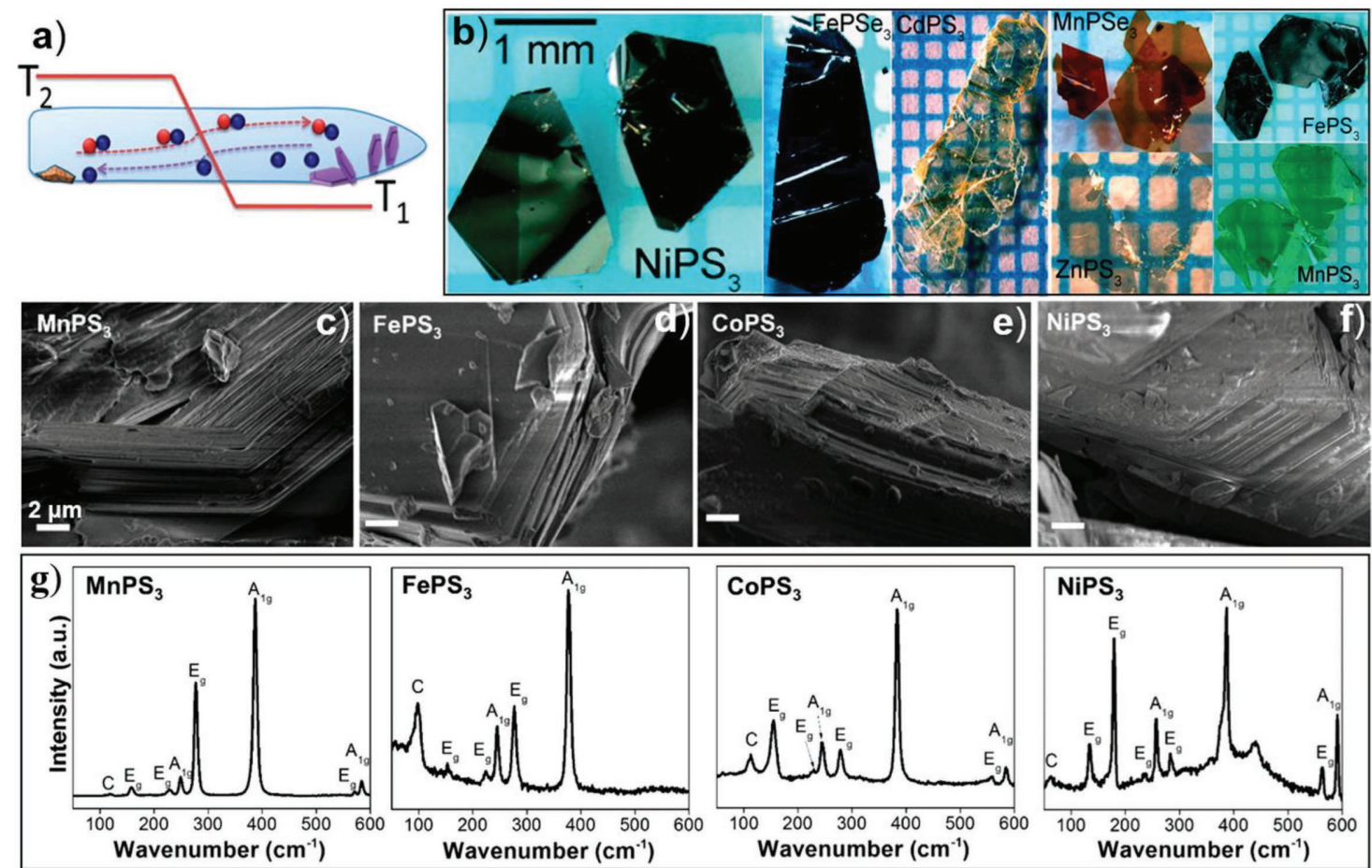

Figure 15. a) Schematics of typical chemical vapor transport (CVT) set-up. b) Photos of bulk 2D crystals obtained via the CVT method. Reproduced with permission. ${ }^{[20]}$ Copyright 2016, American chemical society. c-f) SEM images and g) Raman spectra of various samples grown via the CVT method. Reproduced with permission. ${ }^{[66]}$ Copyright 2017, American chemical society. 

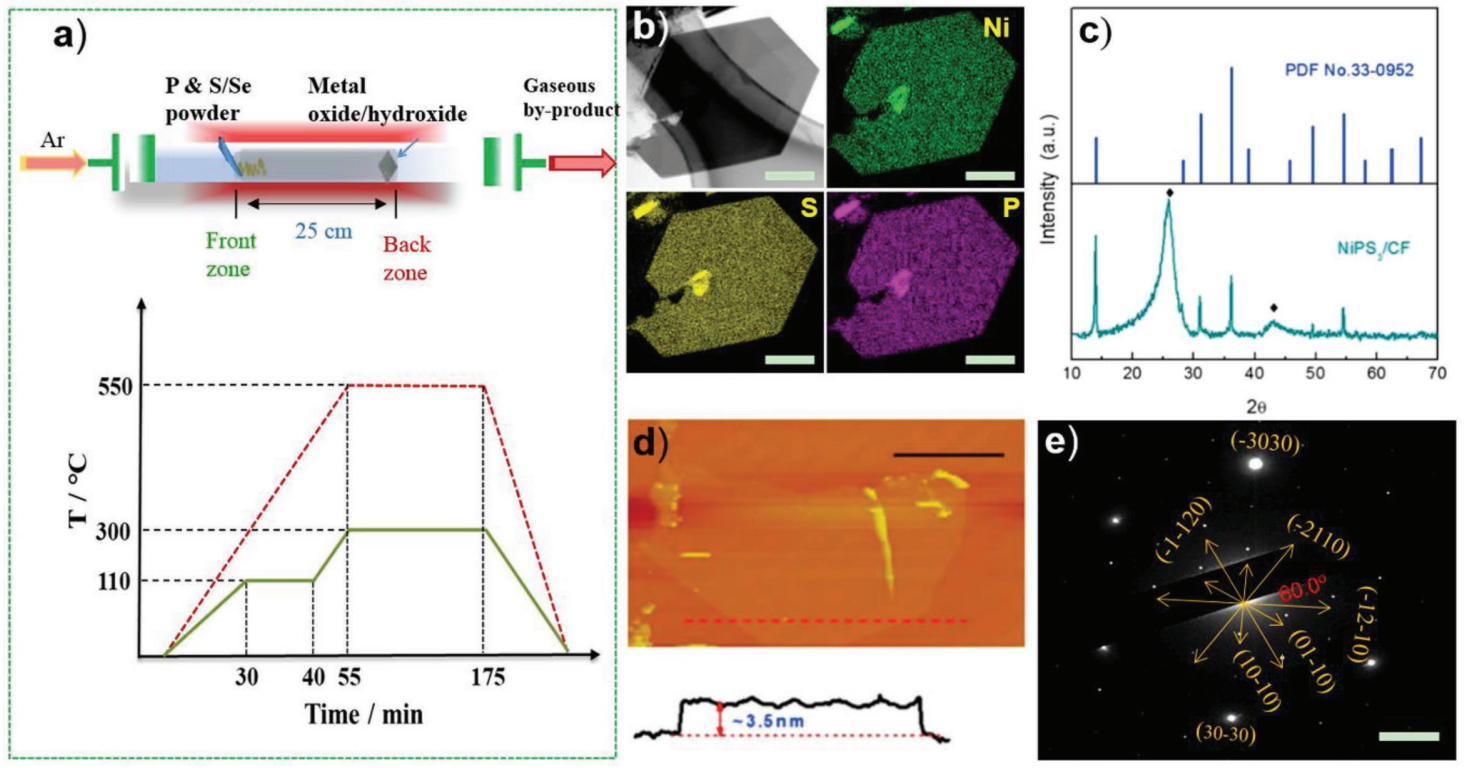

Figure 16. a) Schematics of typical two zone chemical vapor deposition setup (top) and temperature profile in which a preheating strategy is implemented (bottom). b) EDX elemental mapping, c) XRD pattern, and d) AFM image of ultrathin $\mathrm{NiPS}_{3}$ grown according to the schematic shown in (a). e) Selected area electron diffraction pattern of hexagonal $\mathrm{MnPSe}_{3}$ nanosheet grown by a preheating strategy. Scale bars: (b) $=500 \mathrm{~nm},(\mathrm{~d})=5 \mu \mathrm{m}$, (e) $=2 \mathrm{~nm}^{-1}$. (b)-(d) Reproduced with permission. ${ }^{[36]}$ Copyright 2017, Elsevier. (a), (e) Reproduced with permission. ${ }^{[106]}$ Copyright 2018, Wiley-VCH.

The equilibrium condition for the product dissociation can also be reached, which limits the practical completion of the reaction. Thus, merely raising the temperature cannot lead to a faster growth route.

A more strategic approach in vapor phase growth is the chemical vapor deposition (CVD) method, in which the chemical constituents first heated to vapor phase and then reacted. The desired product is obtained through controlled reaction of one or more volatile precursors on the surface of suitable substrate. In this situation, volatile by-products are also frequently formed and they can be removed by flowing gas in the reaction, chamber as shown in Figure 16a (top). As far as the growth of $\mathrm{MPX}_{3}$ materials is concerned, CVD is a very recent technique that enabled the controllable synthesis for various members to fit to the desired applications. A pregrown metal oxide/hydroxide precursor as a metal source is placed at the back zone to meet the incoming vapors of phosphorus and chalcogen from the front zone. The temperature in the front zone requires careful optimization to assure simultaneous vaporization of both $\mathrm{P}$ and $\mathrm{S} / \mathrm{Se}$ sources, which otherwise results in the introduction of impure phases. The temperature is held constant for $\approx 10 \mathrm{~min}$, as shown in Figure 16a (bottom), for this purpose. Very recently, He and co-workers reported the synthesis of ultrathin $\mathrm{NiPS}_{3}$ nanosheets on a carbon fiber substrate from $\mathrm{Ni}(\mathrm{OH})_{2}$ nanosheets, red phosphorous, and sulfur using this approach. ${ }^{[36]}$ Reaction time and temperature play substantial roles in tuning the morphology of the incoming product. Initially, small and irregular sheets with sizes below $500 \mathrm{~nm}$ were formed. With the increasing of the reaction time, the product increased in lateral size and the nanosheets were formed. Moreover, the low temperature (below $400{ }^{\circ} \mathrm{C}$ ) was found to be inefficient to complete the reaction and, hence, ruminants of unreacted hydroxides were observed, whereas a high temperature (above $500{ }^{\circ} \mathrm{C}$ ) caused etching on the surface of the nanosheet. Given the optimized temperature and time of reaction, the obtained nanosheet has the thickness of $\leq 3.5 \mathrm{~nm}$ with the lateral size of larger than $15 \mu \mathrm{m}$. And all the elements $(\mathrm{Ni}, \mathrm{P}$, and $\mathrm{S})$ distributed uniformly throughout the material (Figure 16b,d). It is also obvious that the X-ray diffraction peaks match the standard $\mathrm{NiPS}_{3}$ crystallographic pattern, as is depicted in Figure 16c. Using this approach, the independent growth of metal chalcogenide may occur along with the growth of the desired $\mathrm{MPX}_{3}$, in some cases. This problem introduces impure phases and defies the realization of controllable growth. A preheating strategy, which assures the formation of fused mixture of chalcogen and phosphorous, can tackle this problem. After preheating, the mixed vapor can subsequently be carried to the back zone to meet the metal precursors, as depicted in the temperature profile of the growth route (Figure 16a, bottom). The growth made through this approach yielded high crystal quality of $\mathrm{MnPSe}_{3}$ and $\mathrm{MnPS}_{3}$ nanosheets (Figure 16f). ${ }^{[106]}$ Thus, careful optimization of the temperature profile in CVD confers a facile and successful conversion of oxide/hydroxide precursor to phospho-trichalcogenide with phase purity and high crystal quality.

\section{Potential Applications of $\mathrm{MPX}_{3}$ Compounds}

\subsection{Catalysis}

The $\mathrm{MPX}_{3}$ materials have received significant attention because of their unique physical and chemical characteristics, which are mainly originated from their energy band model and electronic structure. This peculiar structure endows the $\mathrm{MPX}_{3}$ materials with excellent magnetic, electronic, optical, and catalytic 

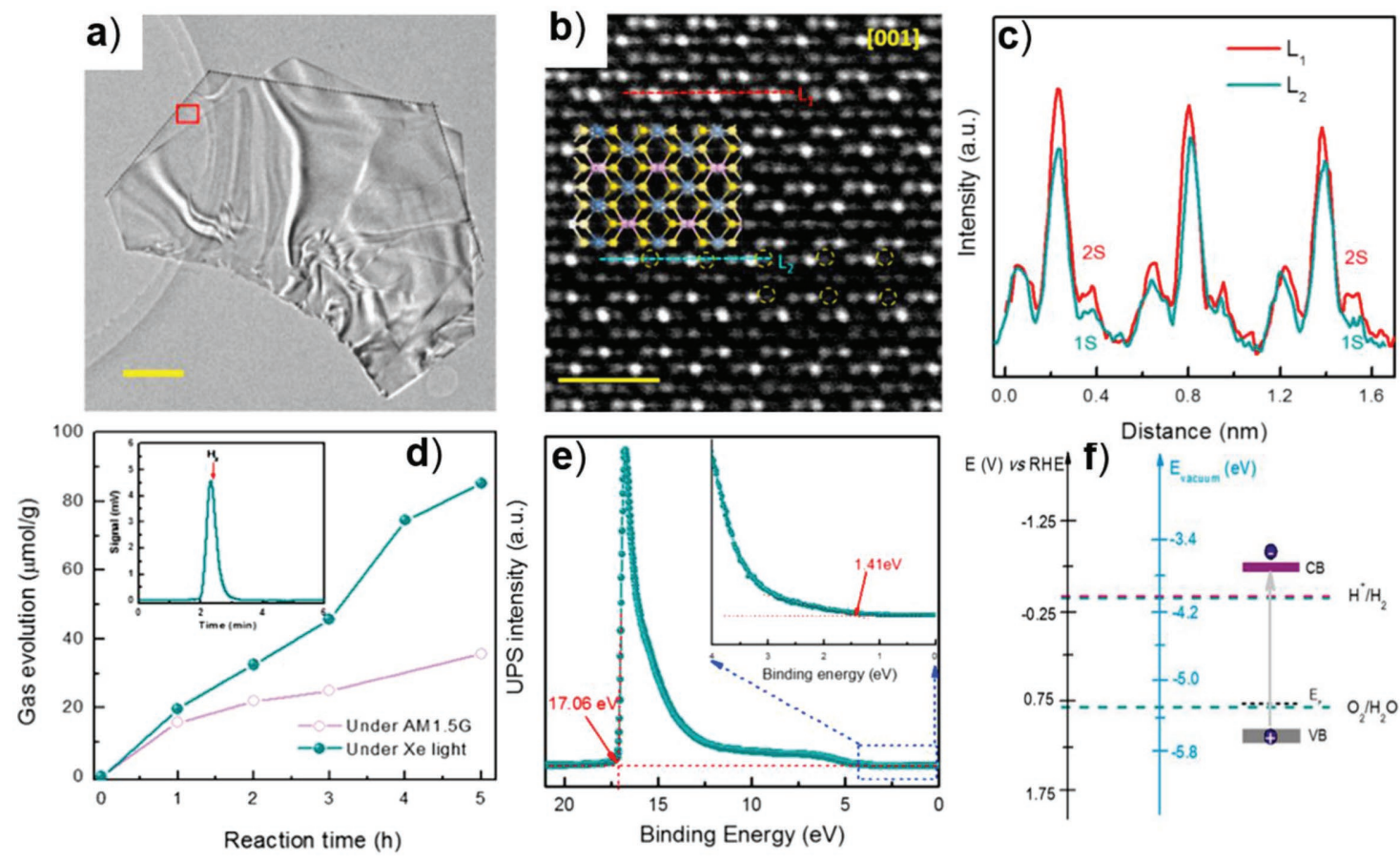

Figure 17. a) Low-magnification TEM image of a hexagonal 2D NiPS ${ }_{3}$ crystal. b) Atomic-level HAADF-STEM image of an ultrathin NiPS ${ }_{3}$ nanosheet showing the sulfur vacancies (yellow circles). c) Intensity profiles along lines L1-L2. Higher contract is obtained from the Ni atom compared to the S atom. d) The comparison of $\mathrm{H}_{2}$ production from pure water under different light irradiation. Inset: A typical gas chromatogram (GC) trace of evolved hydrogen gas after $10 \mathrm{~h}$ with irradiation of Xe-Light. e) UPS spectra of $\mathrm{NiPS}_{3}$ crystals. f) Band structure diagram for NiPS ${ }_{3}$ nanosheets. VB: valence band. CB: conduction band. Scale bar, (a) $1 \mu \mathrm{m}$, (b) $1 \mathrm{~nm}$. Reproduced with permission. ${ }^{[36]}$ Copyright 2017, Elsevier Ltd.

properties. ${ }^{[21,30,107]}$ The wide bandgaps $(1.3-3.5 \mathrm{eV})$ and super colossal specific surface area of $\mathrm{MPX}_{3}$ materials can result in the wide spectrum light absorption, outstanding interfacial reaction activity in photoelectronic properties, and photocatalytic activities. Considering these fascinating properties, the attention of different research groups has been devoted to the rational design of photo (electro) catalysts based on $\mathrm{MPX}_{3}$ compounds. The experimental realization for photo-electrochemical property of $\mathrm{SnPS}_{3}, \mathrm{FePS}_{3}$, and $\mathrm{NiPS}_{3}$ was made in early 1980 s on their bulk states. ${ }^{[25,108]}$ Recently, Wang et al. successfully synthesized high crystalline quality 2D structured $\mathrm{NiPS}_{3}$ nanosheets with thickness of a few atomic layers and large lateral sizes up to tens of micrometers (Figures 16b-d and $17 \mathrm{a}-\mathrm{c}) .{ }^{[36]}$ The atom resolved high angle annular dark-field scanning transmission electron microscopy (HAADF-STEM) image shows that sulfur vacancies $(7.6 \%)$ are observed in favor of creating more defective catalytic sites. Interestingly, the $\mathrm{NiPS}_{3}$ nanosheets can realize water-splitting without sacrificial agent to produce hydrogen gas. In this system, the $\mathrm{H}_{2}$ evolution rates are $\approx 26.42$ and $6.46 \mu \mathrm{mol} \mathrm{g}^{-1} \mathrm{~h}^{-1}$ under Xe light and simulated solar light, respectively (Figure 17d). Furthermore, a proper bandgap is of primary importance for photocatalytic reactions. Based on the optical absorption spectrum, MottSchottky plots, and ultraviolet photoelectron spectroscopy, the conduction band energy $\left(-5.57 \mathrm{eV}\right.$ vs $\left.E_{\text {vacuum }}\right)$ of the $\mathrm{NiPS}_{3}$ nanosheets is placed above the water reduction $\left(\mathrm{H}_{2} / \mathrm{H}_{2} \mathrm{O}\right)$ potential (Figure 17e), which is in a thermodynamically efficient position to generate $\mathrm{H}_{2}$ gas from water. The valence band energy $(-3.61 \mathrm{eV})$ is slightly below the oxidation level of $\mathrm{H}_{2} \mathrm{O}$ to
$\mathrm{O}_{2}$ and the presence of sulfur vacancies will suppress the generation of $\mathrm{O}_{2}$ gas via forming strong adsorption with an oxygen intermediate species (Figure 17f). These intermediates can be traced by a spin trapping experiment based on electron spin resonance spectroscopy, which detects the surface hydroxyl radicals. ${ }^{[06]}$ This finding reveals that photogenerated holes would oxidize the $\mathrm{H}_{2} \mathrm{O}$ molecules adsorbed on the surface of the photocatalysts to hydroxyl radicals, and the road to $\mathrm{O}-\mathrm{O}$ covalent bond formation for oxygen molecules has not been completed. Thus, much more research on the photocatalytic properties of $\mathrm{MPX}_{3}$ nanosheets should be conducted to realize the overall water splitting activity with excellent performance.

2D $\mathrm{MPX}_{3}$ materials are also utilized as electrocatalysts in hydrogen evolution reaction (HER), oxygen evolution reaction (OER), and oxygen reduction reaction, presently. ${ }^{[66,100,105]}$ Their electocatalytic activity results from the appreciable conductivity and abundant active sites at room temperature. ${ }^{[99,105]}$ More significantly, the atomic-level thickness of the $\mathrm{MPX}_{3}$ materials not only enlarges the specific surface area to expose more active sites but also increases the intrinsic catalytic activity. Song et al. ${ }^{[105]}$ synthesized the bulk materials and corresponding 2D ultrathin nanosheets of $\mathrm{Ni}_{1-x} \mathrm{Fe}_{x} \mathrm{PS}_{3}(x=0,0.05,0.1,0.15,1)$ via ultrasonication-assisted exfoliation. The SEM and transmission electron microscopy (TEM) images of exfoliated $\mathrm{Ni}_{0.9} \mathrm{Fe}_{0.1} \mathrm{PS}_{3}$ nanosheets show several micrometers in the lateral size and clear lattice fringes (Figure 18a-c). The average thickness of the exfoliated $\mathrm{Ni}_{0.9} \mathrm{Fe}_{0.1} \mathrm{PS}_{3}$ nanosheets is found to be $4 \mathrm{~nm}$ (Figure 18d), indicating that a single nanosheet consists of approximately five layers. The electrochemical tests show that 

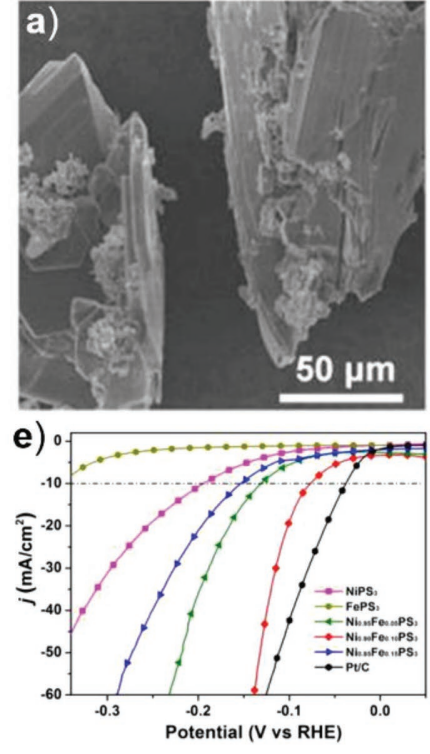
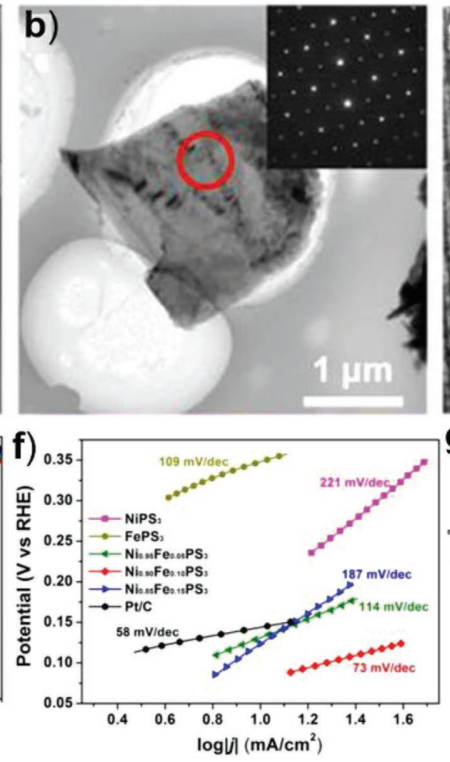
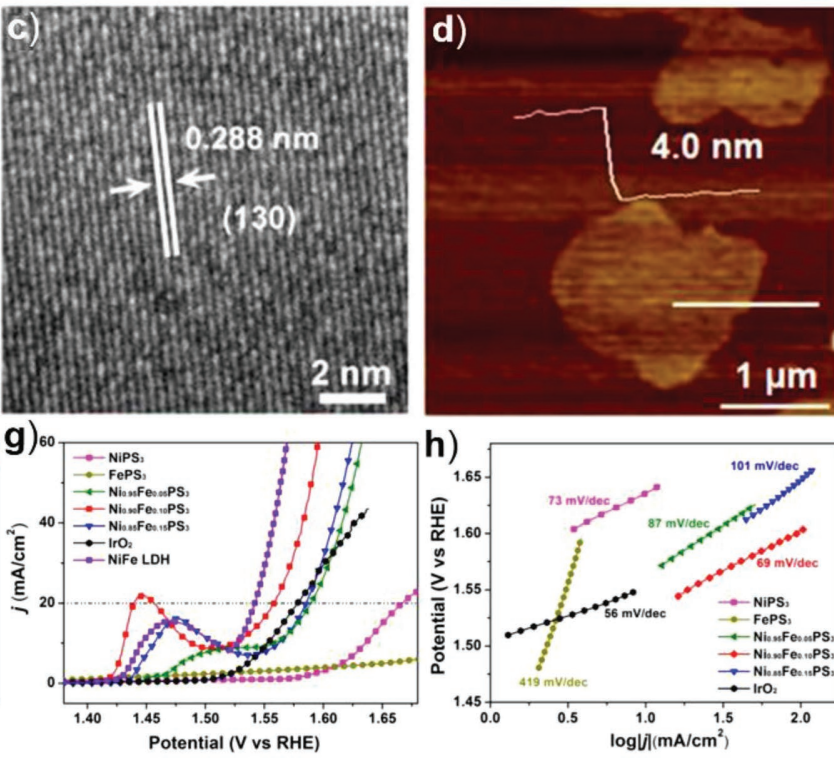

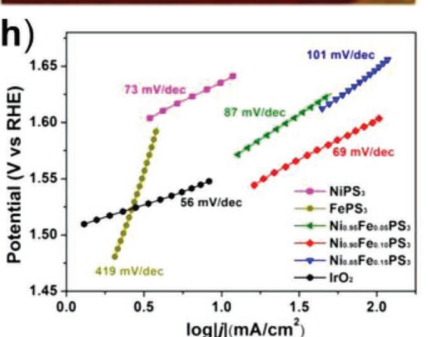

Figure 18. a) $\mathrm{SEM}$ image of bulk $\mathrm{Ni}_{0.9} \mathrm{Fe}_{0.1} \mathrm{PS}_{3}$ crystals. b) TEM image of $\mathrm{NiPS}_{3}$ nanosheets. Inset: SAED in d). c) HRTEM image of $\mathrm{Ni}_{0.9} \mathrm{Fe}_{0.1} \mathrm{PS}{ }_{3}$ along the [001] zone axis. d) AFM images of the as-exfoliated $\mathrm{Ni}_{0.9} \mathrm{Fe}_{0.1} \mathrm{PS}_{3}$ nanosheets. e) $\mathrm{J}-\mathrm{V}$ curves after $i \mathrm{R}$ correction of FePS $\mathrm{NiPS}_{3}$, and various $\mathrm{Ni}_{1-x} \mathrm{Fe}_{x} \mathrm{PS}_{3}$ electrocatalysts in comparison to $20 \mathrm{wt} \% \mathrm{Pt} / \mathrm{C}$ commercial catalyst. f) Tafel plots for the data presented in (e). g) $\mathrm{J}-\mathrm{V}$ curves and $\mathrm{h}$ ) Tafel plots after $\mathrm{R}$ correction of OER performance of $\mathrm{FePS}_{3}, \mathrm{NiPS}_{3}$, and various $\mathrm{Ni}_{1-x} \mathrm{Fe}_{x} \mathrm{PS}_{3}$ samples in comparison to IrO ${ }_{2}$ and $\mathrm{NiFe}^{\mathrm{LDH}}$. Reproduced with permission. ${ }^{[100]}$ Copyright 2017, American Chemical Society.

the $\mathrm{FePS}_{3}$ and $\mathrm{NiPS}_{3}$ nanosheets have much poorer electrocatalytic activities compared to the exfoliated $\mathrm{Ni}_{1-x} \mathrm{Fe}_{x} \mathrm{PS}_{3}(x=0.05$, $0.1,0.15)$ nanosheets that exhibited extremely high HER and OER activity and durability in the aqueous solution of $1 \mathrm{M} \mathrm{KOH}$ (Figure $18 \mathrm{e}-\mathrm{h}$ ). This is attributable to the increase of intrinsic conductivity and electrochemical active surface area. Interestingly, the results show that the $\mathrm{Ni}_{0.9} \mathrm{Fe}_{0.1} \mathrm{PS}_{3}$ nanosheets, whose surface would be transformed into hydroxides as the real active species, serve as the OER electrocatalysts in alkaline media.

\subsection{Electrochemical Storage}

Despite being a very promising future green energy source, hydrogen suffers from the lack of efficient and safe storage systems. Layered compounds, in general, are thought to advance the technology of hydrogen storage owing to their ability to be reversibly intercalated with the corresponding ions. Promising performances were reported using $\mathrm{MPX}_{3}$ compounds such as $\mathrm{MnPS}_{3},{ }^{[109]} \mathrm{NiPS}_{3},{ }^{[24]}$ and $\mathrm{FePS}_{3}{ }^{[104]}$ Hydrogen is assumed to be adsorbed on the surface, in the micropores, and in the interlayer spacing. The hydrogen sorption capacity over $\mathrm{MnPS}_{3}$ at different temperatures and pressures can be observed from the work by Ismail et al. in Figure 19a. ${ }^{[109]} \mathrm{MnPS}_{3}$ is found to exhibit an adsorption capacity of $0.7 \mathrm{wt} \%$ within $100 \mathrm{~min}$ and the same duration is required to desorb it completely. The capacity reaches to $2.9 \mathrm{wt} \%$ at an elevated pressure of $30 \mathrm{bar}$ in $30 \mathrm{~min}$; however, in this case, a longer duration is required for desorption. The hydrogen adsorption/desorption cycles do not affect their crystal structure, but the overall performance is altered by the crystallinity of the material. The effect of crystallinity of the $\mathrm{FePS}_{3}$ powders in hydrogen sorption at $193{ }^{\circ} \mathrm{C}$ is illustrated in Figure 19b. ${ }^{[104]}$ A linear progression can be observed up to 20 bar in the hydrogen sorption isotherm wherein the hydrogen sorption capacity for single crystalline and polycrystalline $\mathrm{FePS}_{3}$ is recorded as $2.2 \mathrm{wt} \%$ and $1.7 \%$, respectively (Figure 19b). A well-ordered single crystalline phase and wider interplanar space are usually associated with better performance for hydrogen sorption efficiency. ${ }^{[104]}$ These findings emphasize the favorability of $\mathrm{MPX}_{3}$ compounds for hydrogen storage and, hence, many other members can be explored following these promising performances.

$\mathrm{MPX}_{3}$ compounds find substantial application in secondary $\mathrm{Li} / \mathrm{Na}$ ion batteries due to their ability to create sufficient paths of lithium ion and exhibit high electronic conductivity with excellent ions mobility (Section 1.2.5). A particularly advantageous feature of this family in lithium batteries emanates from the broader van der Waals gap compared to TMDs, which make the unit cell parameter of the $\mathrm{MPX}_{3}$ compounds remain unaffected after lithium intercalation. ${ }^{[29,110]}$ Therefore, they provide enough capacity for ions while maintaining structure stable during the charge-discharge cycles. It has also been suggested that the very good $\mathrm{Li} / \mathrm{Na}$ ion storage for battery application is following the electrochemical reaction involving nine lithium atoms per molecule of $\mathrm{MPX}_{3}$ intercalation, as follows ${ }^{[22,87,111]}$

$\mathrm{MPX}_{3}+9 \mathrm{Li}^{+}+9 \mathrm{e}^{-} \rightarrow 3 \mathrm{Li}_{2} \mathrm{X}+\mathrm{Li}_{3} \mathrm{P}+\mathrm{M}$

The formation of lithium (the same works for sodium) phosphides and sulfides endows a better conductivity compared to the lithium/sodium oxides that are commonly formed from the metal oxide materials. ${ }^{[23,86]}$ Thus, this family is expected to demonstrate a better rate and cycling performance with high specific capacity. The sufficient electron conduction and paths for lithium ions were corroborated via employing $\mathrm{FePS}_{3}$ materials for lithium ion battery application. ${ }^{[112]}$ In this set up, a 

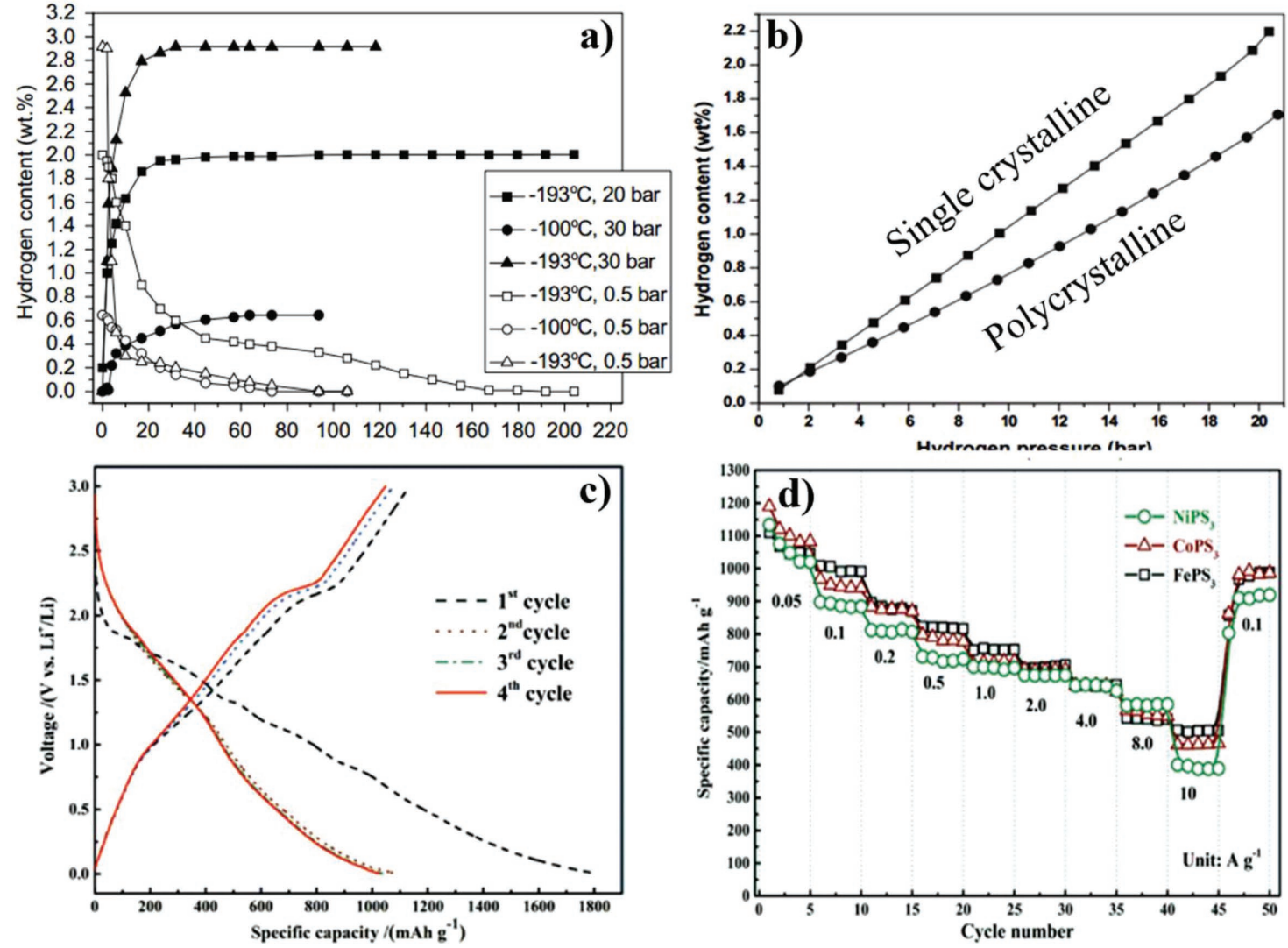

Figure 19. a) Kinetics curves of $\mathrm{MnPS}_{3}$ powders for $\mathrm{H}_{2}$ sorption at different temperatures and pressures. Reproduced with permission. [109]

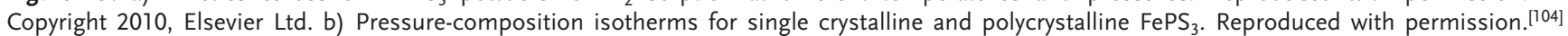
Copyright 2010, Elsevier Ltd. c) Galvanostatic charge-discharge profiles of $\mathrm{NiPS}_{3}$ nanosheet at $0.05 \mathrm{~A} \mathrm{~g}^{-1}$ and d) rate capabilities of MPS 3 (M $=\mathrm{Fe}$, Co, Ni). Reproduced with permission. ${ }^{[23]}$ Copyright 2017, Wiley-VCH.

reversible charge-discharge processes under $0.13 \mathrm{~mA} \mathrm{~cm}^{-2}$ at room temperature was demonstrated. Furthermore, the work by Yan and co-workers ${ }^{[23]}$ is a typical experimental report demonstrating the $\mathrm{Li} / \mathrm{Na}$ ions storage properties of $\mathrm{FePS}_{3}, \mathrm{NiPS}_{3}$, and $\mathrm{CoPS}_{3}$ nanosheets. The clean surface, high crystallinity, high surface area, and better contact with the electrolyte or current collector endow superior performance. They exhibit significantly better performance of Li-ion/Na-ion storage compared to their bulk $\left.(<300 \mathrm{~mA} \mathrm{~h} \mathrm{~g})^{-1}\right)^{[111,112]}$ or sulfide counterparts $\left(<1000 \mathrm{~mA} \mathrm{~h} \mathrm{~g}^{-1}\right) \cdot{ }^{[113]}$ The ultrathin nanosheets shorten the diffusion paths and accelerate the charge transfer process leading to a discharge and charge capacity of 1796 and $1100 \mathrm{~mA} \mathrm{~h} \mathrm{~g}^{-1}$ at a current density of $0.05 \mathrm{~A} \mathrm{~g}^{-1}$, respectively (Figure 19c), with an initial Coulombic efficiency (CE) of $61.2 \%$. The CE reaches almost $100 \%$ after the third cycle due to the minimized loss of capacity from side reactions, such as electrolyte decomposition and solid-electrolyte interphase formation. Collectively, the fourth cycle specific capacities at current densities of 0.05 and $10 \mathrm{~A} \mathrm{~g}^{-1}$ can be gleaned from Figure 19d as 1026 and $383 \mathrm{~mA} \mathrm{~h} \mathrm{~g}^{-1}\left(\mathrm{NiPS}_{3}\right), 1046$ and $506 \mathrm{~mA} \mathrm{~h} \mathrm{~g}{ }^{-1}\left(\mathrm{FePS}_{3}\right)$, and 1086 and $465 \mathrm{~mA} \mathrm{~h} \mathrm{~g}^{-1}\left(\mathrm{CoPS}_{3}\right)$, respectively.

On top of their intrinsic property, the improvement with respect to $\mathrm{Li} / \mathrm{Na}$ storage performance is more pronounced by employing different strategies such as polymer intercalation, hybrid structure formation, etc. The effect of polymer intercalation in these materials has been investigated through intercalation with propylamine. In this case, the interlayer distance becomes further enlarged to allow easier ion accessibility and faster ion diffusion. ${ }^{[23]}$ Hybrid structure formation also plays its own role in enhancing the performance as exhibited in $\mathrm{Li}_{2} \mathrm{~S}-\mathrm{FePS} \mathrm{S}_{3}$ composites where it appears to endow structural reversibility against the $\mathrm{Li}$ extraction/insertion reactions. ${ }^{[114]}$ Moreover, elemental doping along with the formation of $0 \mathrm{D}$ and 2D nanohybrids has recently been reported ${ }^{[115]}$ to elucidate the collective role of bimetallic $\mathrm{MPX}_{3}\left(\mathrm{Co}_{0.5} \mathrm{Ni}_{0.5} \mathrm{PS}_{3}\right)$ and graphene in $\mathrm{Li}$ ion storage. The electrochemical performance is associated with the insertion of $\mathrm{Li}$ ions into the layered space of the $\left(\mathrm{Co}_{0.5} \mathrm{Ni}_{0.5} \mathrm{PS}_{3}\right)$ lattice, which is accompanied by gradual reduction of $\mathrm{Co}^{2+}$ and $\mathrm{Ni}^{2+}$ to elemental $\mathrm{Co}$ and $\mathrm{Ni}$; and the formation of $\mathrm{Li}_{2} \mathrm{~S}$. The graphene support offers a conductive scaffold which contributes to the improved stability and rate performance.

\subsection{Other Applications}

The continual effort to explore the fascinating properties of metal phosphorus trichalcogenides also applies to many other applications. These materials are acknowledged for their high carrier mobility. ${ }^{[21]}$ The recent experimental work by Lee et al. ${ }^{[16]}$ demonstrates the thickness dependent tunneling transport properties 

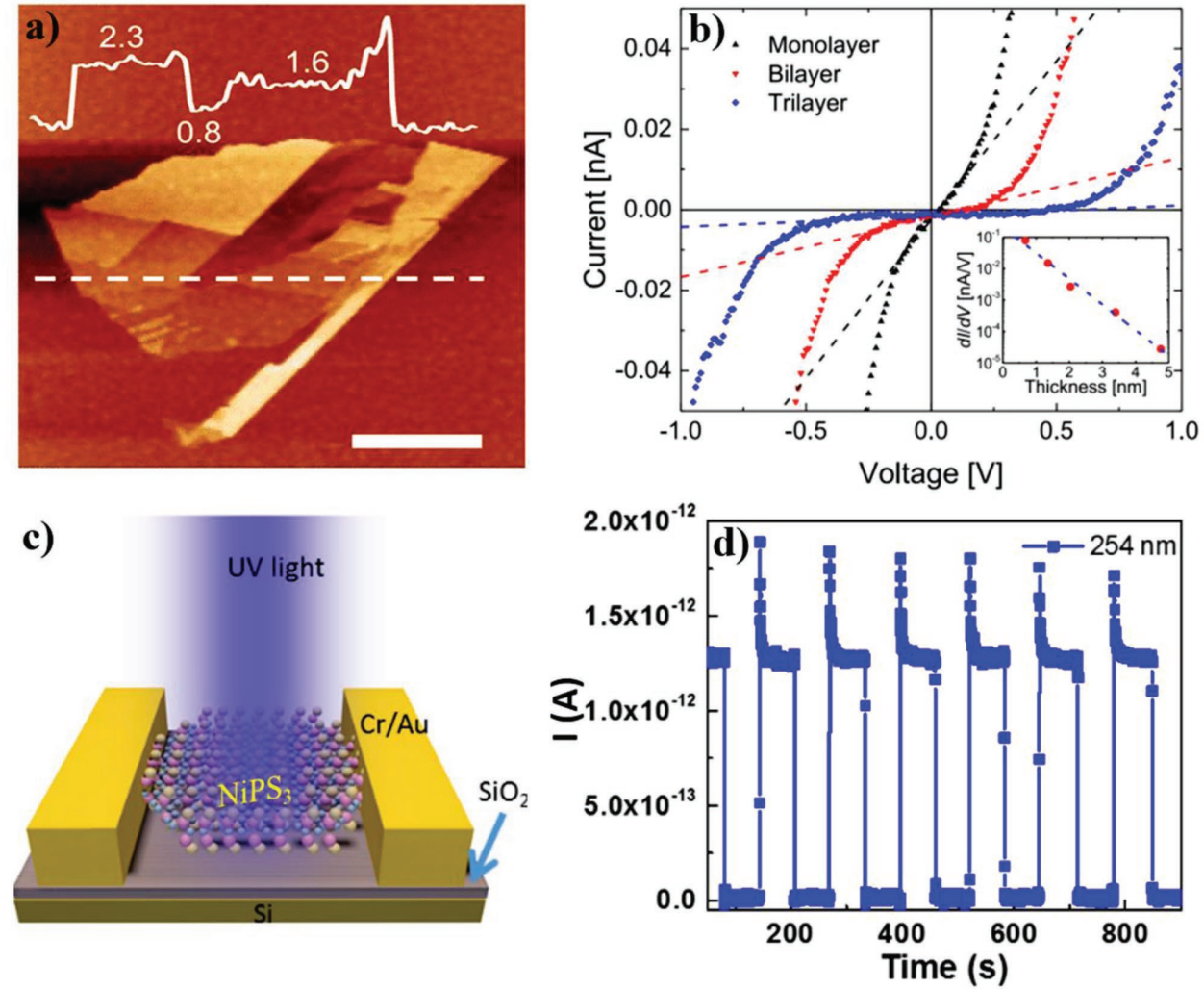

Figure 20. a) AFM images depicting various thicknesses (monolayer to 6 layers) of $\mathrm{MnPS}_{3}$ obtained via scotch-tape exfoliation of the CVT product $($ scale bar $=5 \mu \mathrm{m})$. b) $I-V$ data at a low voltage region of monolayer, bilayer, and trilayer $\mathrm{MnPS}_{3}$. The inset indicates that the slope of the linear behavior at low voltage decreases exponentially as the number of layers increases. Reproduced with permission. ${ }^{[16]}$ Copyright 2016, AIP. c) A schematic and d) time-dependent photoresponse representing the applicability of ultrathin $\mathrm{NiPS}_{3}$ as a promising UV photodetector. Reproduced with permission. ${ }^{[61]}$ Copyright 2017, Wiley-VCH.

of $\mathrm{MnPS}_{3}$. Flakes of various thicknesses, including mono layer and tens of layers (AFM image, Figure 20a), were obtained via the CVT method followed by scotch-tape exfoliation. A Schottky junction tunneling behavior was observed across the indium tin oxides (ITO) $/ \mathrm{MnPS}_{3} / \mathrm{Pt}$-Ir junction with a dielectric breakdown strength of $5.41 \mathrm{mV} \mathrm{cm}^{-1}$. Figure 20b shows that the conductance depends on the number of layers, which exponentially decreases with the increase of the thickness (i.e., the number of layers), as illustrated in the inset of Figure 20b. Moreover, the availability of ample elemental choices in the $\mathrm{MPX}_{3}$ family enables the adjustment of bandgaps in the range of 1.3-3.5 eV (from near infrared to the UV region). This wide range spectrum suggests their optoelectronic application in a broad wavelength horizon. Recently, high photodetectivity of $1.22 \times 10^{12}$ Jones and ultrafast rise times shorter than $5 \mathrm{~ms}$ have been observed ${ }^{[61]}$ from ultrathin $\mathrm{NiPS}_{3}$ (Figure 20c,d). The ease of integration with the Si substrate and the better performance comparable with a commercial GaN UV detector can initiate the investigation of many others in this family. It is, therefore, compelling to mention that the members in the family of $\mathrm{MPX}_{3}$ can find many outstanding applications in various fields due to their unique properties compared to other 2D materials. For instance, the cleavage energy required for thinning their bulk to the atomic thickness level is smaller compared to that of graphite. This makes the fabrication of monolayer heterostructures by van der Waals stacking easier, paving the way to the realization of unusual ferroelectric and magnetic properties. ${ }^{[20,95]}$ Thus, it is interesting to note that the variation in metal components or in chalcogen components provides a conducive platform for the study of the incoming differences in spin-electron, electronic, or optoelectronic devices.

\section{Future Perspectives}

Layered $\mathrm{MPX}_{3}$ compounds constitute members that spread over a wide range of the periodic table. The $\mathrm{M}^{\mathrm{II}}, \mathrm{M}^{\mathrm{I}}$, and $\mathrm{M}^{\mathrm{III}}$ states are stably located in octahedral sulfur or selenium environments, which is a notable difference compared to TMDs, whose cationic components are in the form of the $\mathrm{M}^{\mathrm{IV}}$ state. The strong ionic bond between $\mathrm{M}$ cations and $\left[\mathrm{P}_{2} \mathrm{X}_{6}\right]^{4-}$ endows $\mathrm{MPX}_{3}$ materials with rich functionalities. These configuration features give rise to the unique chemical and physical properties as well as the key distinction from TMDs. Alteration in the size of metal atoms $\left(\mathrm{M}^{\mathrm{II}}\right)$, electronegativity, and electronic configuration can be used to study the structural, catalytic, and magnetic variation of the phases. As to the substituted phases of $\mathrm{M}^{\mathrm{I}} \mathrm{M}^{\mathrm{III}} \mathrm{PX}_{3}$, they present different cationic ordering from the original which is more pronounced in magnetism and ferroelectricity due to the cationic radius size ratio.

In this review, we have discussed the relation between crystal structure and component variations, unique properties, 
and progress in growth, characterization, and applications of $\mathrm{MPX}_{3}$ crystals. The current growth status reveals the successful realization of ultrathin $\mathrm{NiPS}_{3}$ and $\mathrm{MnPX}_{3}$ nanosheets via the chemical vapor deposition method. It is reasonable to say that the growth of the $\mathrm{MPX}_{3}$ monolayer, similar to the TMD monolayer, has yet to be thoroughly explored and prepared. More efforts should be devoted to the controllable growth of $\mathrm{MPX}_{3}$. As mentioned, ionic bonding also contributes to the comparatively large bandgap range from 1.3 to $3.5 \mathrm{eV}$, which is unavailable for other 2D materials, indicating their potential application in optoelectronics and photo(electro)chemical catalysis. Another fascinating future direction would be designing heterostructures using $\mathrm{MPX}_{3}$ as a component part and exploring the promising applications therein. At the same time, we envision that the magnetic 2D $\mathrm{MPX}_{3}$ nanosheets play an indispensable role in spintronic devices. Their magnetism, especially the monolayer's, needs to be comprehensively studied via experimental methods in the 2D limit. Meanwhile, this rich magnetism and spin ordering in the electronic structure can find a promising future in catalysis. With the application of external magnetic field, the carrier mobility may be accelerated thereby boosting the catalytic properties. The high ions mobility and electronic transfer process are crucial for lithium and sodium batteries. Considering the general preparation approach, tunable interlayer spacing, and the ultrathin feature, such 2D MPX 3 crystals have great potential in energy conversion.

\section{Acknowledgements}

F.M.W. and T.A.S. contributed equally to this work. This work was supported by the National Natural Science Foundation of China (Grant Nos.6162540, 61474033, 61574050, 11674072, and 21703047), the Ministry of Science and Technology of China (Grant No. 2016YFA0200700), the Strategic Priority Research Program of the Chinese Academy of Sciences (Grant No. XDA09040201), and the CAS Key Laboratory of Nanosystem and Hierarchical Fabrication. The authors also gratefully acknowledge the support of the Youth Innovation Promotion Association CAS. X.L. and F.X. are grateful for the support of the National Natural Science Foundation of China (Grant Nos. 21525523 and 21722507).

\section{Conflict of Interest}

The authors declare no conflict of interest.

\section{Keywords}

2D materials, current developments, metal phosphorus trichalcogenides, potential application, unique properties

Received: March 27, 2018

Revised: June 4, 2018

Published online:

[1] a) K. S. Novoselov, V. I. Falko, L. Colombo, P. R. Gellert, M. G. Schwab, K. Kim, Nature 2012, 490, 192; b) Q. Bao, K. P. Loh, ACS Nano 2012, 6, 3677; c) Y. Kim, S. S. Cruz, K. Lee,
B. O. Alawode, C. Choi, Y. Song, J. M. Johnson, C. Heidelberger, W. Kong, S. Choi, K. Qiao, I. Almansouri, E. A. Fitzgerald, J. Kong, A. M. Kolpak, J. Hwang, J. Kim, Nature 2017, 544, 340; d) A. S. de Wijn, Nature 2016, 539, 502.

[2] D. Ovchinnikov, F. Gargiulo, A. Allain, D. J. Pasquier, D. Dumcenco, C. H. Ho, O. V. Yazyev, A. Kis, Nat. Commun. 2016, 7, 12391.

[3] a) V. Nicolosi, M. Chhowalla, M. G. Kanatzidis, M. S. Strano, J. N. Coleman, Science 2013, 340, 1226419; b) S.-Y. Xu, I. Belopolski, N. Alidoust, M. Neupane, G. Bian, C. Zhang, R. Sankar, G. Chang, Z. Yuan, C. C. Lee, S. M. Huang, H. Zheng, J. Ma, D. S. Sanchez, B. Wang, A. Bansil, F. Chou, P. P. Shibayev, H. Lin, S. Jia, M. Z. Hasan, Science 2015, 349, 613; c) Z. Ye, T. Cao, K. O. Brien, H. Zhu, X. Yin, Y. Wang, S. G. Louie, X. Zhang, Nature 2014, 513, 214; d) W. Xu, W. Liu, J. F. Schmidt, W. Zhao, X. Lu, T. Raab, C. Diederichs, W. Gao, D. V. Seletskiy, Q. Xiong, Nature 2016, 541, 62

[4] a) F. Xia, H. Wang, Y. Jia, Nat. Commun. 2014, 5, 4458; b) H. Yuan, X. Liu, F. Afshinmanesh, W. Li, G. Xu, J. Sun, B. Lian, A. G. Curto, G. Ye, Y. Hikita, Z. Shen, S. C. Zhang, X. Chen, M. Brongersma, H. Y. Hwang, Y. Cui, Nat. Nanotechnol. 2015, 10, 707.

[5] a) C. T. Wee, L. Huang, J. N. Rui, L. Wang, N. H. Dihan Md, J. D. Thorin, S. K. Karuppannan, A. N. Christian, C. Lee, K. W. Ang, Adv. Mater. 2017, 30, 1705039; b) M. Buscema, D. J. Groenendijk, S. I. Blanter, G. A. Steele, H. S. J. van der Zant, A. CastellanosGomez, Nano Lett. 2014, 14, 3347; c) R. Fei, L. Yang, Nano Lett. 2014, 14, 2884.

[6] a) K. Watanabe, T. Taniguchi, H. Kanda, Nat. Mater. 2004, 3, 404; b) G. Cassabois, P. Valvin, B. Gil, Nat. Photonics 2016, 10, 262; c) Y. Kubota, K. Watanabe, O. Tsuda, T. Taniguchi, Science 2007, 317, 932; d) M. Schnell, A. García-Etxarri, A. J. Huber, K. Crozier, J. Aizpurua, R. Hillenbrand, Nat. Photonics 2009, 3, 287; e) M. Yankowitz, J. Xue, D. Cormode, J. D. Sanchez-Yamagishi, K. Watanabe, T. Taniguchi, P. Jarillo-Herrero, P. Jacquod, B. J. LeRoy, Nat. Phys. 2012, 8, 382; f) W. Yang, G. Chen, Z. Shi, C. C. Liu, L. Zhang, G. Xie, M. Cheng, D. Wang, R. Yang, D. Shi, K. Watanabe, T. Taniguchi, Y. Yao, Y. Zhang, G. Zhang, Nat. Mater. 2013, 12, 792; g) Z. Liu, L. Ma, G. Shi, W. Zhou, Y. Gong, S. Lei, X. Yang, J. Zhang, J. Yu, K. P. Hackenberg, A. Babakhani, J. C. Idrobo, R. Vajtai, J. Lou, P. M. Ajayan, Nat. Nanotechnol. 2013, 8, 119; h) S. Dai, Z. Fei, Q. Ma, A. S. Rodin, M. Wagner, A. S. McLeod, M. K. Liu, W. Gannett, W. Regan, K. Watanabe, T. Taniguchi, M. Thiemens, G. Dominguez, A. H. C. Neto, A. Zettl, F. Keilmann, P. Jarillo-Herrero, M. M. Fogler, D. N. Basov, Science 2014, 343, 1125.

[7] a) K. S. Novoselov, A. Mishchenko, A. Carvalho, A. H. Castro Neto, Science 2016, 353, 461; b) G. Fiori, F. Bonaccorso, G. Iannaccone, T. Palacios, D. Neumaier, A. Seabaugh, S. K. Banerjee, L. Colombo, Nat. Nanotechnol. 2014, 9, 768; c) L. Zhang, P. Bampoulis, A. N. Rudenko, Q. Yao, A. van Houselt, B. Poelsema, M. I. Katsnelson, H. J. W. Zandvliet, Phys. Rev. Lett. 2016, 116, 256804; d) A. A. Tonkikh, E. N. Voloshina, P. Werner, H. Blumtritt, B. Senkovskiy, G. Güntherodt, S. S. P. Parkin, Y. S. Dedkov, Sci. Rep. 2016, 6, 23547; e) Y. Liu, N. O. Weiss, X. Duan, H. C. Cheng, Y. Huang, X. Duan, Nat. Rev. Mater. 2016, 1, 16042.

[8] a) P. Goli, J. Khan, D. Wickramaratne, R. K. Lake, A. A. Balandin, Nano Lett. 2012, 12, 5941; b) X. Xi, L. Zhao, Z. Wang, H. Berger, L. Forró, J. Shan, K. F. Mak, Nat. Nanotechnol. 2015, 10, 765.

[9] a) X. Wang, A. M. Jones, K. L. Seyler, V. Tran, Y. Jia, H. Zhao, H. Wang, L. Yang, X. Xu, F. Xia, Nat. Nanotechnol. 2015, 10, 517; b) M. A. McGuire, G. Clark, S. Kc, W. M. Chance, G. E. Jellison, V. R. Cooper, X. Xu, B. C. Sales, Phys. Rev. Mater. 2017, 1, 014001.

[10] H. Cai, B. Chen, G. Wang, E. Soignard, A. Khosravi, M. Manca, X. Marie, L. Y. Chang Shery, B. Urbaszek, S. Tongay, Adv. Mater. 2017, 29, 1605551. 
[11] M. Chhowalla, H. S. Shin, G. Eda, L. J. Li, K. P. Loh, H. Zhang, Nat. Chem. 2013, 5, 263.

[12] a) T. Roy, M. Tosun, X. Cao, H. Fang, D. H. Lien, P. Zhao, Y. Z. Chen, Y. L. Chueh, J. Guo, A. Javey, ACS Nano 2015, 9, 2071 b) S. Kawai, A. S. Foster, T. Björkman, S. Nowakowska, J. Björk, F. F. Canova, L. H. Gade, T. A. Jung, E. Meyer, Nat. Commun. 2016, 7, 11559; c) X. Wang, F. Xia, Nat. Mater. 2015, 14, 264.

[13] K. F. Mak, C. Lee, J. Hone, J. Shan, T. F. Heinz, Phys. Rev. Lett. 2010, 105, 136805

[14] a) M. J. Allen, V. C. Tung, R. B. Kaner, Chem. Rev. 2010, 110, 132; b) Y. Liu, X. Hua, C. Xiao, T. Zhou, P. Huang, Z. Guo, B. Pan, Y. Xie, J. Am. Chem. Soc. 2016, 138, 5087; c) F. Wang, Y. Li, A. Shifa Tofik, K. Liu, F. Wang, Z. Wang, P. Xu, Q. Wang, J. He, Angew. Chem., Int. Ed. 2016, 128, 7033; d) L. Yang, H. Hong, Q. Fu, Y. Huang, J. Zhang, X. Cui, Z. Fan, K. Liu, B. Xiang, ACS Nano 2015, 9, 6478.

[15] Y. Li, Y.-L. Li, C. M. Araujo, W. Luo, R. Ahuja, Catal. Sci. Technol. 2013, 3, 2214

[16] J. Kang, S. Tongay, J. Zhou, J. Li, J. Wu, Appl. Phys. Lett. 2013, 102, 012111

[17] C. Friedel, Compt. Rend. 1894, 119, 260.

[18] G. Ouvrard, R. Brec, J. Rouxel, Mater. Res. Bull. 1985, 20, 1181.

[19] L. Ferrand, Bull. Soc. Chie. Fr. 1895, 13, 115.

[20] K. z. Du, X. z. Wang, Y. Liu, P. Hu, M. I. B. Utama, C. K. Gan, Q. Xiong, C. Kloc, ACS Nano 2016, 10, 1738.

[21] X. Zhang, X. Zhao, D. Wu, Y. Jing, Z. Zhou, Adv. Sci. 2016, 3, 1600062

[22] Y. V. Kuzminskii, B. M. Voronin, N. N. Redin, J. Power Sources 1995, 55, 133.

[23] Q. Liang, Y. Zheng, C. Du, Y. Luo, J. Zhang, B. Li, Y. Zong, Q. Yan, Small Methods 2017, 1, 1700304.

[24] N. Ismail, M. Madian, A. A. El-Meligi, J. Alloys Compd. 2014, 588, 573.

[25] C. E. Byvik, B. Reichman, D. W. Coleman, J. Electrochem. Soc. 1982 129, 237.

[26] G. Le Flem, R. Brec, G. Ouvard, A. Louisy, P. Segransan, J. Phys. Chem. Solids 1982, 43, 455.

[27] K. Kurosawa, S. Saito, Y. Yamaguchi, J. Phys. Soc. Jpn. 1983, 52, 3919.

[28] X. Li, X. Wu, J. Yang, J. Am. Chem. Soc. 2014, 136, 11065.

[29] R. Brec, Solid State lonics 1986, 22, 3.

[30] A. Susner Michael, M. Chyasnavichyus, A. McGuire Michael, P. Ganesh, P. Maksymovych, Adv. Mater. 2017, 29, 1602852.

[31] G. Kliche, Z. Naturforsch., A: Phys., Phys. Chem., Kosmophys. 1983, 38A, 1133.

[32] J. Banys, J. Macutkevic, R. Grigalaitis, J. Vysochanskii, Solid State Ionics 2008, 179, 79.

[33] X. Yan, X. Chen, J. Qin, Mater. Res. Bull. 2011, 46, 235.

[34] a) Y. Vysochanskii, M. Medulych, A. Molnar, K. Glukhov, A. Dziaugys, J. Banys, R. Yevych, M. Maior, Ferroelectrics 2014, 462, 117; b) B. Zapeka, M. Kostyrko, I. Martynyuk-Lototska, R. Vlokh, Philos. Mag. 2015, 95, 382.

[35] J. U. Lee, S. Lee, J. H. Ryoo, S. Kang, T. Y. Kim, P. Kim, C. H. Park, J. G. Park, H. Cheong, Nano Lett. 2016, 16, 7433.

[36] F. Wang, T. A. Shifa, P. He, Z. Cheng, J. Chu, Y. Liu, Z. Wang, F. Wang, Y. Wen, L. Liang, J. He, Nano Energy 2017, 40, 673.

[37] M. Z. Jandali, G. Eulenberger, H. Hahn, Z. Anorg. Allg. Chem. 2004, 447, 105

[38] F. Fournier, J. Berthelot, Y. L. Pascal, Tetrahedron 1984, 40, 339.

[39] A. Wiedenmann, J. Rossat-Mignod, A. Louisy, R. Brec, J. Rouxel, Solid State Commun. 1981, 40, 1067.

[40] S. Jörgens, A. Mewis, Z. Anorg. Allg. Chem. 2003, 630, 51.

[41] R. Brec, G. Ouvrard, A. Louisy, J. Rouxel, Ann. Chim. 1980, 5, 499.

[42] S. Lee, P. Colombet, G. Ouvrard, R. Brec, Inorg. Chem. 1988, 27, 1291.

[43] Z. Ouili, A. Leblanc, P. Colombet, J. Solid State Chem. 1987, 66, 86.
[44] V. Maisonneuve, V. B. Cajipe, A. Simon, R. Von Der Muhll, J. Ravez, Phys. Rev. B 1997, 56, 10860.

[45] V. Maisonneuve, M. Evain, C. Payen, V. B. Cajipe, P. Molinié, J. Alloys Compd. 1995, 218, 157.

[46] G. Burr, E. Durand, M. Evain, R. Brec, J. Solid State Chem. 1993 , 103, 514.

[47] R. Shannon, Acta Crystallogr., Sect. A 1976, 32, 751.

[48] M. A. Gave, D. Bilc, S. D. Mahanti, J. D. Breshears, M. G. Kanatzidis, Inorg. Chem. 2005, 44, 5293.

[49] R. Brec, D. M. Schleich, G. Ouvrard, A. Louisy, J. Rouxel, Inorg. Chem. 1979, 18, 1814.

[50] Y. Ohno, K. Hirama, J. Solid State Chem. 1986, 63, 258.

[51] F. S. Khumalo, H. P. Hughes, Phys. Rev. B 1981, 23, 5375.

[52] H. Mercier, Y. Mathey, E. Canadell, Inorg. Chem. 1987, 26, 963.

[53] V. Zhukov, S. Alvarez, D. Novikov, J. Phys. Chem. Solids 1996, $57,647$.

[54] B. L. Chittari, Y. Park, D. Lee, M. Han, A. H. MacDonald, E. Hwang, J. Jung, Phys. Rev. B 2016, 94, 184428.

[55] N. Kurita, K. Nakao, J. Phys. Soc. Jpn. 1987, 56, 4455.

[56] Y. M. Vysochanskii, V. A. Stephanovich, A. A. Molnar, V. B. Cajipe, X. Bourdon, Phys. Rev. B 1998, 58, 9119.

[57] a) M. Piacentini, F. S. Khumalo, C. G. Olson, J. W. Anderegg, D. W. Lynch, Chem. Phys. 1982, 65, 289; b) E. J. K. B. Banda, J. Phys. C: Solid State Phys. 1986, 19, 7329.

[58] a) A. C. Ferrari, D. M. Basko, Nat. Nanotechnol. 2013, 8, 235; b) A. Gupta, G. Chen, P. Joshi, S. Tadigadapa, Eklund, Nano Lett. 2006, 6, 2667; c) A. C. Ferrari, J. C. Meyer, V. Scardaci, C. Casiraghi, M. Lazzeri, F. Mauri, S. Piscanec, D. Jiang, K. S. Novoselov, S. Roth, A. K. Geim, Phys. Rev. Lett. 2006, 97, 187401; d) H. Li, Q. Zhang, R. Yap Chin Chong, K. Tay Beng, T. Edwin Teo Hang, A. Olivier, D. Baillargeat, Adv. Funct. Mater. 2012, 22, 1385.

[59] W. Xingzhi, D. Kezhao, L. Yu Yang Fredrik, H. Peng, Z. Jun, Z. Qing, O. Man Hon Samuel, L. Xin, G. Chee Kwan, S. Pinaki, K. Christian, X. Qihua, 2D Mater. 2016, 3, 031009.

[60] C.-T. Kuo, M. Neumann, K. Balamurugan, H. J. Park, S. Kang, H. W. Shiu, J. H. Kang, B. H. Hong, M. Han, T. W. Noh, J.-G. Park, Sci. Rep. 2016, 6, 20904.

[61] J. Chu, F. Wang, L. Yin, L. Lei, C. Yan, F. Wang, Y. Wen, Z. Wang, C. Jiang, L. Feng, J. Xiong, Y. Li, J. He, Adv. Funct. Mater. 2017, 27, 1701342.

[62] a) N. Mounet, M. Gibertini, P. Schwaller, D. Campi, A. Merkys, A. Marrazzo, T. Sohier, I. E. Castelli, A. Cepellotti, G. Pizzi, N. Marzari, Nat. Nanotechnol. 2018, 13, 246; b) N. Miao, B. Xu, L. Zhu, J. Zhou, Z. Sun, J. Am. Chem. Soc. 2018, 140, 2417; c) B. Huang, G. Clark, E. Navarro-Moratalla, D. R. Klein, R. Cheng, K. L. Seyler, D. Zhong, E. Schmidgall, M. A. McGuire, D. H. Cobden, W. Yao, D. Xiao, P. Jarillo-Herrero, X. Xu, Nature 2017, 546, 270; d) K. L. Seyler, D. Zhong, D. R. Klein, S. Gao, X. Zhang, B. Huang, E. Navarro-Moratalla, L. Yang, D. H. Cobden, M. A. McGuire, W. Yao, D. Xiao, P. Jarillo-Herrero, X. Xu, Nat. Phys. 2018, 14, 277; e) S. Jiang, J. Shan, K. F. Mak, Nat. Mater. 2018, 17, 406; f) G. Abellán, C. Martí-Gastaldo, A. Ribera, E. Coronado, Acc. Chem. Res. 2015, 48, 1601; g) M. B. Sanders, K. M. Baroudi, J. W. Krizan, O. A. Mukadam, R. J. Cava, Phys. Status Solidi B 2016, 253, 2056.

[63] C. Felser, H. Fecher Gerhard, B. Balke, Angew. Chem., Int. Ed. 2007, 46, 668.

[64] P. A. Joy, S. Vasudevan, Phys. Rev. B 1992, 46, 5425.

[65] a) S. O. Evans John, D. O'Hare, R. Clement, A. Leaustic, P. Thuéry, Adv. Mater. 2004, 7, 735; b) T. Wataru, M. Toshihiro, W. Tadataka, T. Kouichi, M. Kazuyuki, U. Yoshiya, T. Yoshiki, J. Phys.: Conf. Ser. 2009, 150, 042215; c) D. J. Goossens, Eur. Phys. J. B 2010, 78, 305; d) T. Masubuchi, X. Jin, K. Koyama, Y. Takahashi, K. Takase, Y. Uwatoko, Y. Takano, K. Sekizawa, AIP Conf. Proc. 2006, 850, 1279. 
[66] C. C. Mayorga-Martinez, Z. Sofer, D. Sedmidubský, Š. Huber, A. Y. S. Eng, M. Pumera, ACS Appl. Mater. Interfaces 2017, 9, 12563.

[67] a) E. Ressouche, M. Loire, V. Simonet, R. Ballou, A. Stunault, A. Wildes, Phys. Rev. B 2010, 82, 100408; b) M. A. McGuire, H. Dixit, V. R. Cooper, B. C. Sales, Chem. Mater. 2015, 27, 612; c) A. R. Wildes, K. C. Rule, R. I. Bewley, M. Enderle, T. J. Hicks, J. Phys.: Condens. Matter 2012, 24, 416004; d) T. Asano, Y. Ajiro, M. Mekata, H. Yamazaki, N. Hosoito, T. Shinjo, H. Kikuchi, Solid State Commun. 1994, 90, 125; e) Y. Takano, N. Arai, A. Arai, Y. Takahashi, K. Takase, K. Sekizawa, J. Magn. Magn. Mater. 2004, 272-276, E593.

[68] D. J. Goossens, S. Brazier-Hollins, D. R. James, W. D. Hutchison, J. R. Hester, J. Magn. Magn. Mater. 2013, 334, 82

[69] K. C. Rule, G. J. Mclntyre, S. J. Kennedy, T. J. Hicks, Phys. Rev. B 2007, 76, 134402.

[70] A. R. Wildes, B. Roessli, B. Lebech, K. W. Godfrey, J. Phys.: Condens. Matter 1998, 10, 6417.

[71] N. Chandrasekharan, S. Vasudevan, J. Phys.: Condens. Matter 1994, 6, 4569.

[72] A. R. Wildes, V. Simonet, E. Ressouche, G. J. Mclntyre, M. Avdeev, E. Suard, S. A. J. Kimber, D. Lançon, G. Pepe, B. Moubaraki, T. J. Hicks, Phys. Rev. B 2015, 92, 224408.

[73] a) L. J. Sandilands, J. X. Shen, G. M. Chugunov, S. Y. F. Zhao, S. Ono, Y. Ando, K. S. Burch, Phys. Rev. B 2010, 82, 064503; b) W. H. Ko, Z. X. Liu, T. K. Ng, P. A. Lee, Phys. Rev. B 2010, 81, 024414.

[74] N. Suzuki, H. Kamimura, J Phys. Soc. Jpn. 1973, 35, 985.

[75] D. J. Goossens, T. J. Hicks, J. Phys.: Condens. Matter 1998, 10, 7643.

[76] T. Masubuchi, H. Hoya, T. Watanabe, Y. Takahashi, S. Ban, N. Ohkubo, K. Takase, Y. Takano, J. Alloys Compd. 2008, 460, 668.

[77] J. Macutkevic, J. Banys, R. Grigalaitis, Y. Vysochanskii, Phys. Rev. B 2008, 78, 064101

[78] X. Bourdon, A. R. Grimmer, V. B. Cajipe, Phosphorus Res. Bull. $1999,10,100$

[79] X. Bourdon, V. Maisonneuve, V. B. Cajipe, C. Payen, J. E. Fischer, J. Alloys Compd. 1999, 283, 122.

[80] A. Simon, J. Ravez, V. Maisonneuve, C. Payen, V. B. Cajipe, Chem. Mater. 1994, 6, 1575.

[81] E. Durand, G. Ouvrard, M. Evain, R. Brec, Inorg. Chem. 1990, 29, 4916.

[82] V. Shusta, I. Prits, P. Guranich, E. Gerzanich, A. Slivka, Condens. Matter Phys. 2007, 49, 91.

[83] A. Belianinov, Q. He, A. Dziaugys, P. Maksymovych, E. Eliseev, A. Borisevich, A. Morozovska, J. Banys, Y. Vysochanskii, S. V. Kalinin, Nano Lett. 2015, 15, 3808.

[84] M. A. Susner, A. Belianinov, A. Borisevich, Q. He, M. Chyasnavichyus, H. Demir, D. S. Sholl, P. Ganesh, D. L. Abernathy, M. A. McGuire, P. Maksymovych, ACS Nano 2015, 9, 12365

[85] a) A. H. Thompson, M. S. Whittingham, Mater. Res. Bull. 1977, 12, 741; b) A. Le Méhauté, G. Ouvrard, R. Brec, J. Rouxel, Mater. Res. Bull. 1977, 12, 1191.

[86] X. Y. Yu, L. Yu, W. X. Lou, Adv. Energy Mater. 2015, 6, 1501333.

[87] P. J. S. Foot, T. Katz, S. N. Patel, B. A. Nevett, A. R. Pieecy, A. A. Balchin, Phys. Status. Solidi A 2006, 100, 11.

[88] L. Fabbrizzi, A. Poggi, J. Chem. Soc., Chem. Commun. 1980, 0, 646. [89] W. Klingen, R. Ott, H. Hahn, Z. Anorg. Allg. Chem. 2004, 396, 271.

[90] A. U. Liyanage, M. M. Lerner, RSC Adv. 2012, 2, 474.

[91] K. S. Novoselov, A. K. Geim, S. V. Morozov, D. Jiang, Y. Zhang, S. V. Dubonos, I. V. Grigorieva, A. A. Firsov, Science 2004, 306, 666.

[92] a) J. Zheng, H. Zhang, S. Dong, Y. Liu, C. T. Nai, H. S. Shin, H. Y. Jeong, B. Liu, K. P. Loh, Nat. Commun. 2014, 5, 2995; b) H. Li, J. Wu, Z. Yin, H. Zhang, Acc. Chem. Res. 2014, 47, 1067; c) J. N. Coleman, Acc. Chem. Res. 2013, 46, 14; d) J. Zheng,
H. Zhang, S. Dong, Y. Liu, C. Tai Nai, H. Suk Shin, H. Young Jeong, B. Liu, K. Ping Loh, Nat. Commun. 2014, 5, 2995; e) J. Lee, T. Y. Ko, J. H. Kim, H. Bark, B. Kang, S.-G. Jung, T. Park, Z. Lee, S. Ryu, C. Lee, ACS Nano 2017, 11, 10935; f) C. N. R. Rao, A. Nag, Eur. J. Inorg. Chem. 2010, 2010, 4244; g) K. S. Novoselov, D. Jiang, F. Schedin, T. J. Booth, V. V. Khotkevich, S. V. Morozov, A. K. Geim, Proc. Natl. Acad. Sci. USA 2005, 102, 10451.

[93] J. Liu, X. B. Li, D. Wang, W. M. Lau, P. Peng, L. M. Liu, J. Chem. Phys. 2014, 140, 054707.

[94] a) D. O. Dumcenco, H. Kobayashi, Z. Liu, Y. S. Huang, K. Suenaga, Nat. Commun. 2013, 4, 1351; b) D. J. Late, T. Doneux, M. Bougouma, Appl. Phys. Lett. 2014, 105, 233103; c) H. Li, Z. Yin, Q. He, H. Li, X. Huang, G. Lu, H. Fam Derrick Wen, Y. Tok Alfred ling, Q. Zhang, H. Zhang, Small 2011, 8, 63; d) H. Li, G. Lu, Y. Wang, Z. Yin, C. Cong, Q. He, L. Wang, F. Ding, T. Yu, H. Zhang, Small 2013, 9, 1974; e) J. Late Dattatray, N. Shirodkar Sharmila, V. Waghmare Umesh, P. Dravid Vinayak, C. N. R. Rao, ChemPhysChem 2014, 15, 1592.

[95] Y. Guo, C. Liu, Q. Yin, C. Wei, S. Lin, T. B. Hoffman, Y. Zhao, J. H. Edgar, Q. Chen, S. P. Lau, J. Dai, H. Yao, H. S. P. Wong, Y. Chai, ACS Nano 2016, 10, 8980.

[96] a) V. Goyal, D. Teweldebrhan, A. A. Balandin, Appl. Phys. Lett. 2010, 97, 133117; b) J. Sotor, G. Sobon, W. Macherzynski, P. Paletko, K. Grodecki, K. M. Abramski, Opt. Mater. Express 2014, 4, 1; c) K. M. F. Shahil, M. Z. Hossain, V. Goyal, A. A. Balandin, J. Appl. Phys. 2012, 111, 054305.

[97] P. Ares, F. Aguilar-Galindo, D. Rodríguez-San-Miguel, A. Aldave Diego, S. Diaz-Tendero, M. Alcamf, F. Martín, J. Gómez-Herrero, F. Zamora, Adv. Mater. 2016, 28, 6332.

[98] C. R. Dean, A. F. Young, I. Meric, C. Lee, L. Wang, S. Sorgenfrei, K. Watanabe, T. Taniguchi, P. Kim, K. L. Shepard, J. Hone, Nat. Nanotechnol. 2010, 5, 722.

[99] D. Mukherjee, M. A. P., S. Sampath, ACS Appl. Energy Mater. 2018, 1, 220.

[100] B. Konkena, J. Masa, A. J. R. Botz, I. Sinev, W. Xia, J. Koßmann, R. Drautz, M. Muhler, W. Schuhmann, ACS Catal. 2017, 7, 229.

[101] R. F. Frindt, D. Yang, P. Westreich, J. Mater. Res. 2011, 20, 1107.

[102] D. Mukherjee, P. M. Austeria, S. Sampath, ACS Energy Lett. 2016, 1, 367.

[103] M. Binnewies, R. Glaum, M. Schmidt, P. Schmidt, Z. Anorg. Allg. Chem. 2013, 639, 219.

[104] N. Ismail, A. A. El-Meligi, Y. M. Temerk, M. Madian, Int. J. Hydrogen Energy 2010, 35, 7827.

[105] B. Song, K. Li, Y. Yin, T. Wu, L. Dang, M. Cabán-Acevedo, J. Han, T. Gao, X. Wang, Z. Zhang, J. R. Schmidt, P. Xu, S. Jin, ACS Catal. 2017, 7, 8549 .

[106] A. Shifa Tofik, F. Wang, Z. Cheng, P. He, Y. Liu, C. Jiang, Z. Wang, J. He, Adv. Funct. Mater. 2018, 28, 1800548.

[107] a) M. Y. Lin, L. W. Hourng, C.-W. Kuo, Int. J. Hydrogen Energy 2012, 37, 1311; b) R. Gusmão, Z. Sofer, D. Sedmidubský, Š. Huber, M. Pumera, ACS Catal. 2017, 7, 8159.

[108] C. E. Byvik, B. T. Smith, B. Reichman, Sol. Energy Mater. 1982, 7, 213.

[109] N. Ismail, Y. M. Temerk, A. A. El-Meligi, M. A. Badr, M. Madian, J. Solid State Chem. 2010, 183, 984

[110] J. Rouxel, R. Brec, Annu. Rev. Mater. Sci. 1986, 16, 137.

[111] Y. V. Kuz'minskii, B. M. Voronin, I. M. Petrushina, N. N. Redin, G. P. Prikhodko, J. Power Sources 1995, 55, 1.

[112] Y. Fujii, A. Miura, N. C. Rosero-Navarro, M. Higuchi, K. Tadanaga, Electrochim. Acta 2017, 241, 370.

[113] X. Rui, H. Tan, Q. Yan, Nanoscale 2014, 6, 9889.

[114] T. Takeuchi, H. Kageyama, M. Ogawa, K. Mitsuhara, K. Nakanishi, T. Ohta, A. Sakuda, H. Kobayashi, H. Sakaebe, Z. Ogumi, Solid State lonics 2016, 288, 199.

[115] C. F. Du, Q. Liang, Q. Yan, J. Nat. Gas Chem. 2018, 27, 190. 
[116] S. Lee, K. Y. Choi, S. Lee, B. H. Park, J. G. Park, APL Mater. 2016, 4, 086108.

[117] E. Prouzet, G. Ouvrard, R. Brec, Mater. Res. Bull. 1986, 21, 195

[118] Z. Wang, R. D. Willett, R. A. Laitinen, D. A. Cleary, Chem. Mater. $1995,7,856$

[119] R. Pfeiff, R. Kniep, J. Alloys Compd. 1992, 186, 111.

[120] P. Colombet, A. Leblanc, M. Danot, J. Rouxel, J. Solid State Chem. 1982, 41, 174.

[121] A. Pfitzner, S. Seidlmayer, Z. Anorg. Allg. Chem. 2009, 635, 704.

[122] S. Lee, P. Colombet, G. Ouvrard, R. Brec, Mater. Res. Bull. 1986, 21, 917.

[123] G. Ouvrard, R. Brec, Mater. Res. Bull. 1988, 23, 1199.
[124] R. Pfeiff, R. Kniep, Z. Naturforsch., B 1993, 48, 1270.

[125] I. P. Studenyak, V. V. Mitrovcij, S. Kovacs Gy, M. I. Gurzan, O. A. Mykajlo, M. Vysochanskii Yu, V. B. Cajipe, Phys. Status Solidi B 2003, 236, 678.

[126] C. Calareso, V. Grasso, L. Silipigni, J. Appl. Phys. 1997, 82, 6228.

[127] H. Mutka, C. Payen, P. Molinié, J. L. Soubeyroux, P. Colombet, A. D. Taylor, Phys. Rev. Lett. 1991, 67, 497.

[128] C. Payen, H. Mutka, J. L. Soubeyroux, P. Molinié, P. Colombet, J. Magn. Magn. Mater. 1992, 104-107, 797.

[129] Y. Huang, E. Sutter, N. N. Shi, J. Zheng, T. Yang, D. Englund, H. J. Gao, P. Sutter, ACS Nano 2015, 9, 10612. 